\title{
Tandem Wittig Reaction-Ring Contraction of Cyclobutanes: a Route to Functionalized Cyclopropanecarbaldehydes
}

Federico Cuccu, ${ }^{\text {a }}$ Lorenzo Serusi, ${ }^{\mathrm{a}}$ Alberto Luridiana, ${ }^{\mathrm{a}}$ Francesco Secci, ${ }^{\text {a }}$ Pierluigi Caboni, ${ }^{\mathrm{b}}$ David J. Aitken, ${ }^{\mathrm{c}}$ Angelo Frongia ${ }^{*, \mathrm{a}}$

aDipartimento di Scienze Chimiche e Geologiche, Università degli Studi di Cagliari, Complesso Universitario di Monserrato, S.S. 554, Bivio per Sestu, I-09042, Monserrato, Cagliari, Italy. afrongia@unica.it

bDipartimento di Scienze della Vita e dell'Ambiente, Università degli Studi di Cagliari, Via Ospedale 72, 09124, Cagliari, Italy.

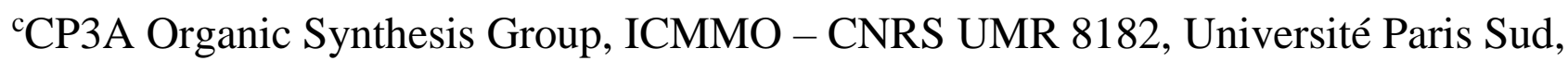
Université Paris Saclay, 15 rue Georges Clemenceau, 91405 Orsay cedex, France.

Table of Contents

1. General methods and analytical data S2-26

2. References S27

3. Copies of ${ }^{1} \mathrm{H}$ and ${ }^{13} \mathrm{C}$ NMR spectra S28-76 


\section{General Methods}

${ }^{1} \mathrm{H}$ NMR spectra were recorded on a Varian 500 spectrometer at ambient temperature with $\mathrm{CDCl}_{3}$ as solvent. Data are reported as follows: chemical shifts $(\delta)$, multiplicity, coupling constants and integration. ${ }^{13} \mathrm{C}$ NMR spectra were recorded operating at 126 $\mathrm{MHz}$ at $27{ }^{\circ} \mathrm{C}$ with $\mathrm{CDCl}_{3}$ as solvent. Infrared spectra were recorded on a FT-IR spectrophotometer. High resolution mass spectra (HRMS) were recorded on a spectrometer using Positive Electro Ionization (ESI) mode. Analytical thin layer chromatography was performed using $0.25 \mathrm{~mm}$ silica gel $60-\mathrm{F}$ plates. Flash chromatography was performed using columns of 230 - 400 mesh silica gel $60(0.040$ - $0.063 \mathrm{~mm}$ ). Compounds $4 \mathbf{4 d}, \mathbf{4 e}, \mathbf{4 f}, \mathbf{4 o}, \mathbf{4 p}, \mathbf{4 r}, \mathbf{4 s}, \mathbf{5 b}, \mathbf{5 c}$ and $\mathbf{5 i}$ were unable to be further purified using standard flash chromatography techniques.

Procedure for preparation of 2-hydroxycyclobutanone (1). ${ }^{1}$<smiles>O=C1CCC1O</smiles>

Prepared following the previously reported procedure starting from commercially available 1,2-bis(trimethylsilyloxy)cyclobutene purchased from Sigma-Aldrich: to a solution of $11.5 \mathrm{~g}(50 \mathrm{mmol})$ of 1,2-bis(trimethylsilyloxy)cyclobutene in $30 \mathrm{~mL}$ of acetone was added successively $1.8 \mathrm{~g}$ of water $(100 \mathrm{mmol})$ and a catalytic amount (50 mg) of $\mathrm{FeCl}_{3} / \mathrm{SiO}_{2}$ (6/100). After stirring at room temperature for $1 \mathrm{~h}$ and elimination of solvent, the residue was filtered through a 2-cm pad of $\mathrm{SiO}_{2}$ eluted with ether $(80 \mathrm{~mL})$. After concentration we obtained $4.25 \mathrm{~g}$ (94\% yield) as viscous oil of pure 2-hydroxycyclobutanone 1, which was used without additional purification. All spectral data were identical with those previously reported.

\section{2-Ethyl-2-hydroxy-cyclobutanone (1'). ${ }^{2}$}

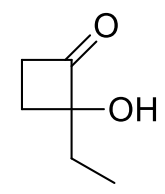

In a $50 \mathrm{~mL}$ round bottom two neck flask, hexane-3,4-dione (4.4 mol) were dissolved in $\mathrm{CH}_{3} \mathrm{CN}(10 \mathrm{~mL})$ and irradiated at $405 \mathrm{~nm}$ (blue LED) for $2-16 \mathrm{~h}\left(25^{\circ} \mathrm{C}\right)$ and followed 
by GC-MS. The organic solutions were evaporated under reduced pressure and purified by flash chromatography (n-hexane/ $\mathrm{Et}_{2} \mathrm{O}, 10: 1-5: 1-3: 1$ ) without previous work-up. Spectral data were in agreement with the literature.

General procedure for preparation of phosphonium ylides (2a-u). ${ }^{3}$<smiles>[R]C(=O)C=P(c1ccccc1)(c1ccccc1)c1ccccc1</smiles>

Aryl or alkyl-substituted 2-bromoethanone (5.00 mmol, 1.0 equiv.) and $\mathrm{PPh}_{3}$ (1.44 g, $5.50 \mathrm{mmol}, 1.1$ equiv.) were refluxed in THF (10 mL) for $4 \mathrm{~h}$. Upon cooling, volatiles were removed, the solids redissolved in DCM and extracted with aq. $\mathrm{NaOH}(20 \% \mathrm{w} / \mathrm{w}$ in $\left.\mathrm{H}_{2} \mathrm{O}, 1 \times 20 \mathrm{~mL}\right)$, washed with water $(1 \times 20 \mathrm{~mL})$, dried over $\mathrm{Na}_{2} \mathrm{SO}_{4}$, filtered and evaporated to dryness to yield the corresponding phosphonium ylide.

Commercially available phosphonium ylides $(\mathbf{2 v - y})$ were obtained from SigmaAldrich and used as received.

General procedure for the preparation of cyclopropanecarbaldehydes $4 a-u$ and cyclobutylidenes $3 w-y$.

A solution of $1(0.05 \mathrm{~g}, 0.58 \mathrm{mmol})$ and phosphonium ylide $2(0.58 \mathrm{mmol})$ in toluene $(0.5 \mathrm{~mL})$ was stirred in a sealed tube reactor at $80{ }^{\circ} \mathrm{C}$ for $48 \mathrm{~h}$. The crude mixture, without aqueous work-up, was directly purified by flash column chromatography (see below for details) to give compound $\mathbf{4 a - u}$ or $\mathbf{3 w}-\mathbf{y}$.

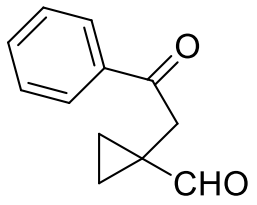

1-(2-oxo-2-phenylethyl)cyclopropanecarbaldehyde (4a): Crude product was purified by flash column chromatography $\left(\mathrm{SiO}_{2}\right.$, petroleum ether/ether $\left.=10: 1 \rightarrow 1: 1\right)$. Yield 82\% (89 mg); orange oil. IR (ATR): 3067, 3010, 2906, 1706, 1689, 1600, 1450, 1410, 1364, 1329, 1277, 1217, $984 \mathrm{~cm}^{-1} .{ }^{1} \mathrm{H}$ NMR (500 MHz, $\left.\mathrm{CDCl}_{3}\right) \delta 8.76(\mathrm{~s}, 1 \mathrm{H})$, $7.97-7.95(\mathrm{~m}, 2 \mathrm{H}), 7.56(\mathrm{t}, J=7.4 \mathrm{~Hz}, 1 \mathrm{H}), 7.46(\mathrm{t}, J=7.7 \mathrm{~Hz}, 2 \mathrm{H}), 3.28(\mathrm{~s}, 2 \mathrm{H})$, 
$1.38(\mathrm{q}, J=4.9 \mathrm{~Hz}, 2 \mathrm{H}), 1.11-1.07(\mathrm{~m}, 2 \mathrm{H}) .{ }^{13} \mathrm{C} \mathrm{NMR}\left(126 \mathrm{MHz}, \mathrm{CDCl}_{3}\right) \delta 200.2$, 197.0, 136.8, 133.3, 128.7, 128.2, 39.5, 28.6, 12.9. HRMS (ESI) Calcd. for $\mathrm{C}_{12} \mathrm{H}_{13} \mathrm{O}_{2}$ $\left(\mathrm{M}+\mathrm{H}^{+}\right) \mathrm{m} / \mathrm{z}$ 189.0910, found 189.0914.

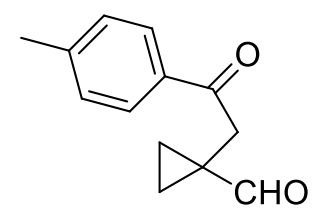

1-(2-oxo-2-(p-tolyl)ethyl)cyclopropanecarbaldehyde (4b): Crude product was purified by flash column chromatography $\left(\mathrm{SiO}_{2}\right.$, petroleum ether/ether $\left.=10: 1 \rightarrow 1: 1\right)$. Yield 76\% (88 mg); yellow solid; m. p. = 86-89 ${ }^{\circ}$ C. IR (ATR): 3091, 3006, 2911, 2862, 1708, 1678, 1605, 1572, 1409, 1368, 1333, 1287, 1224, 1210, 1178, 1036, 987 $\mathrm{cm}^{-1} .{ }^{1} \mathrm{H}$ NMR $\left(500 \mathrm{MHz}, \mathrm{CDCl}_{3}\right) \delta 8.77(\mathrm{~s}, 1 \mathrm{H}), 7.86(\mathrm{~d}, J=8.2 \mathrm{~Hz}, 2 \mathrm{H}), 7.25(\mathrm{~d}, J=$ $8.6 \mathrm{~Hz}, 2 \mathrm{H}), 3.26(\mathrm{~s}, 2 \mathrm{H}), 2.40(\mathrm{~s}, 3 \mathrm{H}), 1.38-1.35(\mathrm{~m}, 2 \mathrm{H}), 1.08-1.05(\mathrm{~m}, 2 \mathrm{H}) .{ }^{13} \mathrm{C}$ NMR (126 MHz, $\left.\mathrm{CDCl}_{3}\right) \delta 200.3,196.7,144.1,134.4,129.4,128.3,39.3,28.5,21.7$, 12.9. HRMS (ESI) Calcd. for $\mathrm{C}_{13} \mathrm{H}_{15} \mathrm{O}_{2}\left(\mathrm{M}+\mathrm{H}^{+}\right) \mathrm{m} / \mathrm{z}$ 203.1066, found 203.1066.<smiles>COc1ccc(C(=O)CC2(C=O)CC2)cc1</smiles>

1-(2-(4-methoxyphenyl)-2-oxoethyl)cyclopropanecarbaldehyde $\quad(4 c): \quad$ Crude product was purified by flash column chromatography $\left(\mathrm{SiO}_{2}\right.$, petroleum ether/ether $=$ $5: 1 \rightarrow 1: 1$ ). Yield 79\% (99 mg); yellow solid; m. p. $=112-115{ }^{\circ} \mathrm{C}$. IR (ATR): 3020, 3006, 2976, 2938, 2908, 1697, 1673, 1602, 1575, 1510, 1458, 1420, 1330, 1251, 1221 , 1170, 1025, $985 \mathrm{~cm}^{-1} .{ }^{1} \mathrm{H} \mathrm{NMR}\left(500 \mathrm{MHz}, \mathrm{CDCl}_{3}\right) \delta 8.76(\mathrm{~s}, 1 \mathrm{H}), 7.93(\mathrm{~d}, J=8.9 \mathrm{~Hz}$, 2H), $6.91(\mathrm{~d}, J=8.9 \mathrm{~Hz}, 2 \mathrm{H}), 3.85(\mathrm{~s}, 3 \mathrm{H}), 3.23(\mathrm{~s}, 2 \mathrm{H}), 1.34(\mathrm{q}, J=4.9 \mathrm{~Hz}, 2 \mathrm{H}), 1.06$ $(\mathrm{q}, J=4.9 \mathrm{~Hz}, 2 \mathrm{H}) .{ }^{13} \mathrm{C} \mathrm{NMR}\left(126 \mathrm{MHz}, \mathrm{CDCl}_{3}\right) \delta 200.4,195.6,163.6,130.4,129.9$, 113.8, 55.5, 39.0, 28.6, 12.9. HRMS (ESI) Calcd. for $\mathrm{C}_{13} \mathrm{H}_{15} \mathrm{O}_{3}\left(\mathrm{M}+\mathrm{H}^{+}\right) \mathrm{m} / \mathrm{z}$ 219.1015, found 219.1015 .

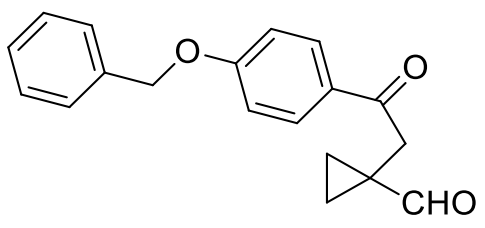


1-(2-(4-(benzyloxy)phenyl)-2-oxoethyl)cyclopropanecarbaldehyde (4d): Crude product was purified by flash column chromatography $\left(\mathrm{SiO}_{2}\right.$, petroleum ether/ether $=$ 5:1 $\rightarrow 1: 1$ ). Yield 85\% (144 mg); white solid; m. p. $=104-106{ }^{\circ} \mathrm{C}$. IR (ATR): 3066, 3036, 3009, 2908, 1706, 1678, 1600, 1575, 1510, 1455, 1420, 1365, 1327, 1259, 1219, 1167, 1012, $985 \mathrm{~cm}^{-1} .{ }^{1} \mathrm{H}$ NMR $\left(500 \mathrm{MHz}, \mathrm{CDCl}_{3}\right) \delta 8.77(\mathrm{~s}, 1 \mathrm{H}), 7.94(\mathrm{~d}, J=8.9 \mathrm{~Hz}$, 2H), $7.41(\mathrm{dt}, J=14.5,7.2 \mathrm{~Hz}, 4 \mathrm{H}), 7.34(\mathrm{t}, J=6.9 \mathrm{~Hz}, 1 \mathrm{H}), 7.01(\mathrm{~d}, J=8.9 \mathrm{~Hz}, 2 \mathrm{H})$, $5.13(\mathrm{~s}, 2 \mathrm{H}), 3.24(\mathrm{~s}, 2 \mathrm{H}), 1.36(\mathrm{q}, J=4.9 \mathrm{~Hz}, 2 \mathrm{H}), 1.07(\mathrm{q}, J=4.9 \mathrm{~Hz}, 2 \mathrm{H}) .{ }^{13} \mathrm{C}$ NMR $\left(126 \mathrm{MHz}, \mathrm{CDCl}_{3}\right) \delta 200.4,195.6,162.8,136.3,130.5,130.1,128.8,128.3,127.6$, 114.7, 70.3, 39.1, 28.6, 13.0. HRMS (ESI) Calcd. for $\mathrm{C}_{19} \mathrm{H}_{19} \mathrm{O}_{3}\left(\mathrm{M}+\mathrm{H}^{+}\right) \mathrm{m} / \mathrm{z} 295.1328$, found 295.1332 .

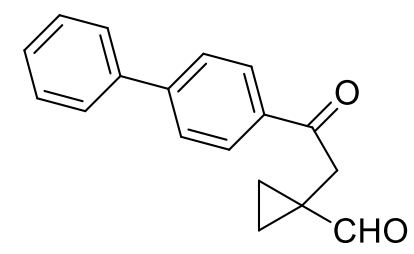

1-(2-([1,1'-biphenyl]-4-yl)-2-oxoethyl)cyclopropanecarbaldehyde (4e): Crude product was purified by flash column chromatography $\left(\mathrm{SiO}_{2}\right.$, petroleum ether/ether $=$ $10: 1 \rightarrow 1: 1$ ). Yield $83 \%$ (127 mg); yellow solid; m. p. $=110-115{ }^{\circ} \mathrm{C}$. IR (ATR): 3088, 3058, 3004, 2906, 1711, 1681, 1602, 1561, 1485, 1406, 1368, 1330, 1268, 1224, 1208, $1115,1034,985 \mathrm{~cm}^{-1} .{ }^{1} \mathrm{H} \mathrm{NMR}\left(500 \mathrm{MHz}, \mathrm{CDCl}_{3}\right) \delta 8.78(\mathrm{~s}, 1 \mathrm{H}), 8.04(\mathrm{~d}, J=8.2 \mathrm{~Hz}$, 2H), $7.69(\mathrm{~d}, J=8.2 \mathrm{~Hz}, 2 \mathrm{H}), 7.63(\mathrm{~d}, J=7.4 \mathrm{~Hz}, 2 \mathrm{H}), 7.47(\mathrm{t}, J=7.5 \mathrm{~Hz}, 2 \mathrm{H}), 7.40$ $(\mathrm{t}, J=7.3 \mathrm{~Hz}, 1 \mathrm{H}), 3.31(\mathrm{~s}, 2 \mathrm{H}), 1.39(\mathrm{t}, J=6.1 \mathrm{~Hz}, 2 \mathrm{H}), 1.11(\mathrm{t}, J=6.0 \mathrm{~Hz}, 2 \mathrm{H}) .{ }^{13} \mathrm{C}$ NMR $\left(126 \mathrm{MHz}, \mathrm{CDCl}_{3}\right) \delta 200.2,196.7,146.0,140.0,135.5,129.0,128.8,128.3$, 127.4, 127.3, 39.5, 28.6, 12.9. HRMS (ESI) Calcd. for $\mathrm{C}_{18} \mathrm{H}_{17} \mathrm{O}_{2}\left(\mathrm{M}+\mathrm{H}^{+}\right) \mathrm{m} / \mathrm{z} 265.1223$, found 265.1223 .

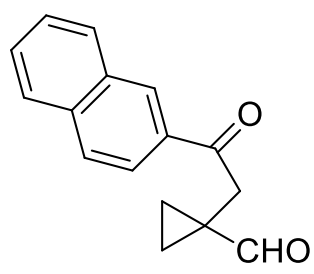

1-(2-(naphthalen-2-yl)-2-oxoethyl)cyclopropanecarbaldehyde (4f): Crude product was purified by flash column chromatography $\left(\mathrm{SiO}_{2}\right.$, petroleum ether/ether = 10:1 $\rightarrow 1: 1$ ). Yield 74\% (102 mg); yellow solid; m. p. $=99-101{ }^{\circ} \mathrm{C}$. IR (ATR): 3096, 5 
$3063,3012,2906,2838,1703,1681,1466,1434,1387,1368,1325,1273,1183,1121$, $990 \mathrm{~cm}^{-1} .{ }^{1} \mathrm{H}$ NMR $\left(500 \mathrm{MHz}, \mathrm{CDCl}_{3}\right) \delta 8.79(\mathrm{~s}, 1 \mathrm{H}), 8.46(\mathrm{~s}, 1 \mathrm{H}), 8.00(\mathrm{dd}, J=8.6$, $1.6 \mathrm{~Hz}, 1 \mathrm{H}), 7.93(\mathrm{~d}, J=8.0 \mathrm{~Hz}, 1 \mathrm{H}), 7.87-7.84(\mathrm{~m}, 2 \mathrm{H}), 7.60-7.56(\mathrm{~m}, 1 \mathrm{H}), 7.55-$ $7.51(\mathrm{~m}, 1 \mathrm{H}), 3.40(\mathrm{~s}, 2 \mathrm{H}), 1.38(\mathrm{q}, J=4.8 \mathrm{~Hz}, 2 \mathrm{H}), 1.11(\mathrm{q}, J=4.8 \mathrm{~Hz}, 2 \mathrm{H}) .{ }^{13} \mathrm{C}$ NMR $\left(126 \mathrm{MHz}, \mathrm{CDCl}_{3}\right) \delta 200.3,197.0,135.8,134.2,132.6,129.9,129.7,128.65,128.63$, 127.9, 126.9, 123.9, 39.5, 28.7, 12.9. HRMS (ESI) Calcd. for $\mathrm{C}_{16} \mathrm{H}_{15} \mathrm{O}_{2}\left(\mathrm{M}+\mathrm{H}^{+}\right) \mathrm{m} / \mathrm{z}$ 239.1066, found 239.1066 .

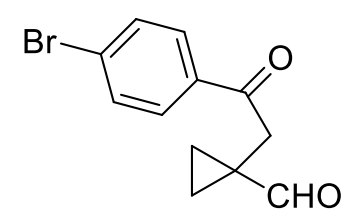

1-(2-(4-bromophenyl)-2-oxoethyl)cyclopropanecarbaldehyde (4g): Crude product was purified by flash column chromatography $\left(\mathrm{SiO}_{2}\right.$, petroleum ether/ether = 5:1 $\rightarrow 1: 1$ ). Yield 75\% (114 mg); orange solid; m. p. $=170-171{ }^{\circ} \mathrm{C}$. IR (ATR): 3096, 3077, 3012, 2995, 2919, 1678, 1659, 1600, 1583, 1395, 1357, 1260, 1213, 1180, 1074, $1006,976,813,764 \mathrm{~cm}^{-1} .{ }^{1} \mathrm{H}$ NMR $\left(500 \mathrm{MHz}, \mathrm{CDCl}_{3}\right) \delta 8.70(\mathrm{~s}, 1 \mathrm{H}), 7.82(\mathrm{~d}, J=8.6$ $\mathrm{Hz}, 2 \mathrm{H}), 7.59$ (d, J = 8.6 Hz, 2H), $3.21(\mathrm{~s}, 2 \mathrm{H}), 1.38-1.36(\mathrm{~m}, 2 \mathrm{H}), 1.11-1.07$ (m, 2H). ${ }^{13} \mathrm{C} \mathrm{NMR}\left(126 \mathrm{MHz}, \mathrm{CDCl}_{3}\right) \delta 200.0,196.1,135.5,132.0,129.7,128.4,39.4$, 28.7, 12.7. HRMS (ESI) Calcd. for $\mathrm{C}_{12} \mathrm{H}_{12} \mathrm{BrO}_{2}\left(\mathrm{M}+\mathrm{H}^{+}\right) \mathrm{m} / \mathrm{z}$ 267.0015, found 267.0013 .

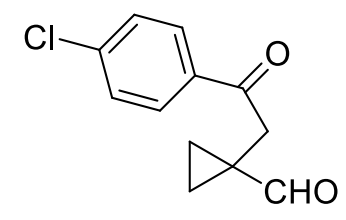

1-(2-(4-chlorophenyl)-2-oxoethyl)cyclopropanecarbaldehyde (4h): Crude product was purified by flash column chromatography $\left(\mathrm{SiO}_{2}\right.$, petroleum ether/ether = 5:1 $\rightarrow 1: 1$ ). Yield 76\% (97 mg); white solid; m. p. $=151-152^{\circ}$ C. IR (ATR): 3088, 3009, 2911, 1711, 1684, 1589, 1572, 1398, 1365, 1330, 1278, 1216, 1175, 1093, 990, 906, 824, 767, $751 \mathrm{~cm}^{-1} .{ }^{1} \mathrm{H}$ NMR $\left(500 \mathrm{MHz}, \mathrm{CDCl}_{3}\right) \delta 8.71(\mathrm{~s}, 1 \mathrm{H}), 7.90(\mathrm{~d}, J=8.6 \mathrm{~Hz}$, 2H), $7.43(\mathrm{~d}, J=8.6 \mathrm{~Hz}, 2 \mathrm{H}), 3.23$ (s, 2H), $1.39-1.37$ (m, 2H), $1.11-1.08$ (m, 2H). ${ }^{13} \mathrm{C} \mathrm{NMR}\left(126 \mathrm{MHz}, \mathrm{CDCl}_{3}\right) \delta 200.0,195.9,139.8,135.2,129.6,129.0,39.4,28.7$, 12.7. HRMS (ESI) Calcd. for $\mathrm{C}_{12} \mathrm{H}_{12} \mathrm{ClO}_{2}\left(\mathrm{M}+\mathrm{H}^{+}\right) \mathrm{m} / \mathrm{z} 223.0520$, found 223.0497 . 


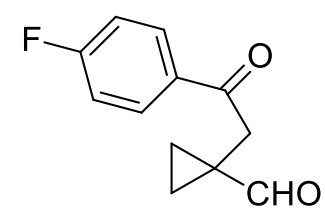

1-(2-(4-fluorophenyl)-2-oxoethyl)cyclopropanecarbaldehyde (4i): Crude product was purified by flash column chromatography $\left(\mathrm{SiO}_{2}\right.$, petroleum ether/ether = 5:1 $\rightarrow 1: 1$ ). Yield 64\% (76 mg); yellow oil. IR (ATR): 3076, 3004, 2906, 1706, 1692, 1597, 1507, 1410, 1366, 1277, 1217, 1159, $992 \mathrm{~cm}^{-1} .{ }^{1} \mathrm{H}$ NMR $\left(500 \mathrm{MHz}, \mathrm{CDCl}_{3}\right) \delta$ $8.71(\mathrm{~s}, 1 \mathrm{H}), 7.99-7.96(\mathrm{~m}, 2 \mathrm{H}), 7.13-7.09$ (m, 2H), 3.22 (s, 2H), 1.36 (q, $J=4.9$ $\mathrm{Hz}, 2 \mathrm{H}), 1.08$ (q, $J=4.9 \mathrm{~Hz}, 2 \mathrm{H}) .{ }^{13} \mathrm{C} \mathrm{NMR}\left(126 \mathrm{MHz}, \mathrm{CDCl}_{3}\right) \delta 200.1,195.4,165.9$ $(\mathrm{d}, J=254.7 \mathrm{~Hz}), 133.2(\mathrm{~d}, J=3.0 \mathrm{~Hz}), 130.8(\mathrm{~d}, J=9.4 \mathrm{~Hz}), 115.8(\mathrm{~d}, J=21.9 \mathrm{~Hz})$, 39.3, 28.6, 12.7. HRMS (ESI) Calcd. for $\mathrm{C}_{12} \mathrm{H}_{12} \mathrm{FO}_{2}\left(\mathrm{M}+\mathrm{H}^{+}\right) \mathrm{m} / \mathrm{z} 207.0815$, found 207.0817.

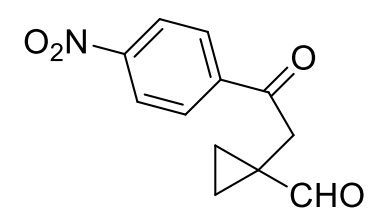

1-(2-(4-nitrophenyl)-2-oxoethyl)cyclopropanecarbaldehyde (4j): Crude product was purified by flash column chromatography $\left(\mathrm{SiO}_{2}\right.$, petroleum ether/ether = 5:1 $\rightarrow 1: 1$ ). Yield 78\% (105 mg); orange semi-solid. IR (ATR): 3112, 3088, 3053, 3012, 2938, 2908, 1700, 1689, 1602, 1518, 1406, 1344, 1319, 1284, 1208, 1112, 1066, 987 $\mathrm{cm}^{-1} .{ }^{1} \mathrm{H}$ NMR $\left(500 \mathrm{MHz}, \mathrm{CDCl}_{3}\right) \delta 8.67(\mathrm{~s}, 1 \mathrm{H}), 8.32-8.30(\mathrm{~m}, 2 \mathrm{H}), 8.13-8.11(\mathrm{~m}$, 2H), $3.26(\mathrm{~s}, 2 \mathrm{H}), 1.42(\mathrm{q}, J=5.1 \mathrm{~Hz}, 2 \mathrm{H}), 1.15(\mathrm{q}, J=5.0 \mathrm{~Hz}, 2 \mathrm{H}) .{ }^{13} \mathrm{C}$ NMR $(126$ $\left.\mathrm{MHz}, \mathrm{CDCl}_{3}\right) \delta 199.7,195.7,150.5,141.3,129.2,123.9,39.9,29.0,12.5$. HRMS (ESI) Calcd. for $\mathrm{C}_{12} \mathrm{H}_{12} \mathrm{NO}_{4}\left(\mathrm{M}+\mathrm{H}^{+}\right) \mathrm{m} / \mathrm{z} 234.0760$, found 234.0760.

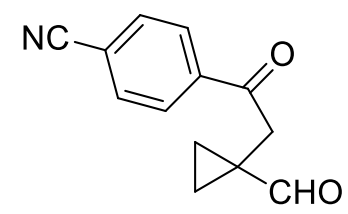

4-(2-(1-formylcyclopropyl)acetyl)benzonitrile (4k): Crude product was purified by flash column chromatography $\left(\mathrm{SiO}_{2}\right.$, petroleum ether/ether $\left.=5: 1 \rightarrow 1: 1\right)$. Yield 80\% $(98$ mg); yellow solid; m. p. = 93-96 ${ }^{\circ} \mathrm{C}$. IR (ATR): 3009, 2955, 2933, 2873, 2326, 1706, 1613, 1469, 1412, 1368, 1276, 1259, 1172, 1134, 1077, 1063, 1034, $995 \mathrm{~cm}^{-1} .{ }^{1} \mathrm{H}$ NMR 
$\left(500 \mathrm{MHz}, \mathrm{CDCl}_{3}\right) \delta 8.65(\mathrm{~s}, 1 \mathrm{H}), 8.04(\mathrm{~d}, J=8.2 \mathrm{~Hz}, 2 \mathrm{H}), 7.76-7.74(\mathrm{~m}, 2 \mathrm{H}), 3.22$ $(\mathrm{s}, 2 \mathrm{H}), 1.39$ (q, $J=5.0 \mathrm{~Hz}, 2 \mathrm{H}), 1.12(\mathrm{q}, J=5.0 \mathrm{~Hz}, 2 \mathrm{H}) .{ }^{13} \mathrm{C} \mathrm{NMR}\left(126 \mathrm{MHz}, \mathrm{CDCl}_{3}\right)$ $\delta$ 199.7, 195.9, 139.8, 132.5, 128.5, 118.0, 116.5, 39.6, 28.8, 12.5. HRMS (ESI) Calcd. for $\mathrm{C}_{13} \mathrm{H}_{12} \mathrm{NO}_{2}\left(\mathrm{M}+\mathrm{H}^{+}\right) \mathrm{m} / \mathrm{z} 214.0862$, found 214.0863.

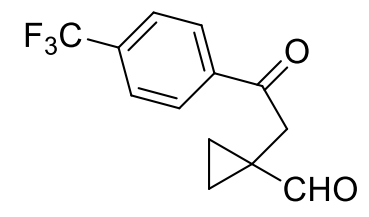

\section{1-(2-oxo-2-(4-(trifluoromethyl)phenyl)ethyl)cyclopropanecarbaldehyde}

(4l):

Crude product was purified by flash column chromatography $\left(\mathrm{SiO}_{2}\right.$, petroleum ether/ether $=5: 1 \rightarrow 1: 1)$. Yield $70 \%$ (104 mg); yellow oil. IR (ATR): 3076, 3010, 2915, 2840, 1692, 1585, 1510, 1410, 1323, 1214, 1171, 1122, 1067, $987 \mathrm{~cm}^{-1} .{ }^{1} \mathrm{H}$ NMR (500 $\left.\mathrm{MHz}, \mathrm{CDCl}_{3}\right) \delta 8.70(\mathrm{~s}, 1 \mathrm{H}), 8.07(\mathrm{~d}, J=8.1 \mathrm{~Hz}, 2 \mathrm{H}), 7.73(\mathrm{~d}, J=8.3 \mathrm{~Hz}, 2 \mathrm{H}), 3.26$ $(\mathrm{s}, 2 \mathrm{H}), 1.40(\mathrm{q}, J=5.3 \mathrm{~Hz}, 2 \mathrm{H}), 1.12(\mathrm{q}, J=5.4 \mathrm{~Hz}, 2 \mathrm{H}) \cdot{ }^{13} \mathrm{C} \mathrm{NMR}\left(126 \mathrm{MHz}, \mathrm{CDCl}_{3}\right)$ $\delta 199.9,196.2,139.5,134.6(\mathrm{q}, J=32.6 \mathrm{~Hz}), 128.5,125.8(\mathrm{q}, J=3.7 \mathrm{~Hz}), 123.7$ (q, $J$ $=272.7 \mathrm{~Hz}$ ), 39.7, 28.8, 12.6. HRMS (ESI) Calcd. for $\mathrm{C}_{13} \mathrm{H}_{12} \mathrm{~F}_{3} \mathrm{O}_{2}\left(\mathrm{M}+\mathrm{H}^{+}\right) \mathrm{m} / \mathrm{z}$ 257.0784, found 257.0782.

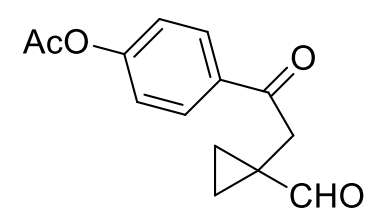

4-(2-(1-formylcyclopropyl)acetyl)phenyl acetate (4m): Crude product was purified by flash column chromatography $\left(\mathrm{SiO}_{2}\right.$, petroleum ether/ether $\left.=10: 1 \rightarrow 1: 1\right)$. Yield $65 \%$ (92 mg); white solid; m. p. $=115-117^{\circ} \mathrm{C}$. IR (ATR): 3063, 3009, 2919, 2851, 1755 , 1706, 1673, 1600, 1502, 1412, 1363, 1333, 1278, 1219, 1194, 1164, 1066, 1036, 1012, $990 \mathrm{~cm}^{-1} .{ }^{1} \mathrm{H}$ NMR $\left(500 \mathrm{MHz}, \mathrm{CDCl}_{3}\right) \delta 8.74(\mathrm{~s}, 1 \mathrm{H}), 8.00(\mathrm{~d}, J=8.6 \mathrm{~Hz}, 2 \mathrm{H}), 7.19(\mathrm{~d}$, $J=8.7 \mathrm{~Hz}, 2 \mathrm{H}), 3.25(\mathrm{~s}, 2 \mathrm{H}), 2.32(\mathrm{~s}, 3 \mathrm{H}), 1.38(\mathrm{q}, J=4.9 \mathrm{~Hz}, 2 \mathrm{H}), 1.09$ (q, $J=4.9$ $\mathrm{Hz}, 2 \mathrm{H}) .{ }^{13} \mathrm{C}$ NMR $\left(126 \mathrm{MHz}, \mathrm{CDCl}_{3}\right) \delta 200.1,195.8,168.9,154.5,134.4,129.8$, 121.9, 39.4, 28.6, 21.2, 12.8. HRMS (ESI) Calcd. for $\mathrm{C}_{14} \mathrm{H}_{15} \mathrm{O}_{4}\left(\mathrm{M}+\mathrm{H}^{+}\right) \mathrm{m} / \mathrm{z} 247.0964$, found 247.0964 . 


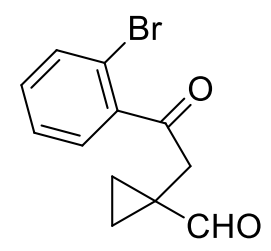

1-(2-(2-bromophenyl)-2-oxoethyl)cyclopropanecarbaldehyde (4n): Crude product was purified by flash column chromatography $\left(\mathrm{SiO}_{2}\right.$, petroleum ether/ether = 5:1 $\rightarrow 1: 1$ ). Yield 55\% (84 mg); yellow oil. IR (ATR): 3082, 3010, 2897, 1700, 1585, $1467,1430,1364,1326,1280,1211,1058,984 \mathrm{~cm}^{-1} .{ }^{1} \mathrm{H}$ NMR $\left(500 \mathrm{MHz}, \mathrm{CDCl}_{3}\right) \delta$ $8.69(\mathrm{~s}, 1 \mathrm{H}), 7.57(\mathrm{dd}, J=8.0,1.0 \mathrm{~Hz}, 1 \mathrm{H}), 7.51(\mathrm{dd}, J=7.6,1.7 \mathrm{~Hz}, 1 \mathrm{H}), 7.37(\mathrm{td}, J$ $=7.5,1.0 \mathrm{~Hz}, 1 \mathrm{H}), 7.30-7.26(\mathrm{~m}, 1 \mathrm{H}), 3.17(\mathrm{~s}, 2 \mathrm{H}), 1.37-1.35(\mathrm{~m}, 2 \mathrm{H}), 1.17-1.14$ $(\mathrm{m}, 2 \mathrm{H}) .{ }^{13} \mathrm{C} \mathrm{NMR}\left(126 \mathrm{MHz}, \mathrm{CDCl}_{3}\right) \delta 201.3,200.0,141.7,133.5,131.6,128.8,127.6$, 118.4, 43.5, 29.2, 12.7. HRMS (ESI) Calcd. for $\mathrm{C}_{12} \mathrm{H}_{12} \mathrm{BrO}_{2}\left(\mathrm{M}+\mathrm{H}^{+}\right) \mathrm{m} / \mathrm{z}$ 267.0015, found 266.9999 .

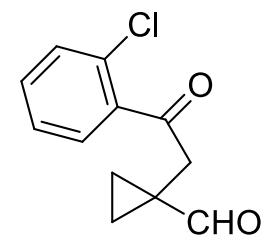

1-(2-(2-chlorophenyl)-2-oxoethyl)cyclopropanecarbaldehyde (4o): Crude product was purified by flash column chromatography $\left(\mathrm{SiO}_{2}\right.$, petroleum ether/ether $=$ 5:1 $\rightarrow 1: 1$ ). Yield 60\% (76 mg); yellow oil. IR (ATR): 3067, 3010, 2900, 1781, 1703, $1588,1473,1433,1364,1283,1214,1067,987 \mathrm{~cm}^{-1} .{ }^{1} \mathrm{H}$ NMR $\left(500 \mathrm{MHz}, \mathrm{CDCl}_{3}\right) \delta$ $8.68(\mathrm{~s}, 1 \mathrm{H}), 7.57-7.55(\mathrm{~m}, 1 \mathrm{H}), 7.41-7.30(\mathrm{~m}, 3 \mathrm{H}), 3.19(\mathrm{~s}, 2 \mathrm{H}), 1.37-1.34(\mathrm{~m}$, 2H), $1.16-1.12(\mathrm{~m}, 2 \mathrm{H}) .{ }^{13} \mathrm{C}$ NMR $\left(126 \mathrm{MHz}, \mathrm{CDCl}_{3}\right) \delta 200.4,200.0,139.4,131.8$, 130.7, 130.4, 129.3, 127.1, 43.8, 29.3, 12.7. HRMS (ESI) Calcd. for $\mathrm{C}_{12} \mathrm{H}_{12} \mathrm{ClO}_{2}$ $\left(\mathrm{M}+\mathrm{H}^{+}\right) \mathrm{m} / \mathrm{z} 223.0520$, found 223.0520.

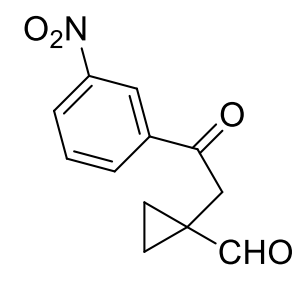

1-(2-(3-nitrophenyl)-2-oxoethyl)cyclopropanecarbaldehyde (4p): Crude product was purified by flash column chromatography $\left(\mathrm{SiO}_{2}\right.$, petroleum ether/ether $=$ 5:1 $\rightarrow 1: 1$ ). Yield $81 \%$ (109 mg); orange solid; m. p. $=98-99^{\circ} \mathrm{C}$. IR (ATR): 3061, 3006, 
2900, 1687, 1662, 1605, 1447, 1355, 1257, 1210, 1180, 1023, $982 \mathrm{~cm}^{-1} .{ }^{1} \mathrm{H}$ NMR (500 $\left.\mathrm{MHz}, \mathrm{CDCl}_{3}\right) \delta 8.77(\mathrm{t}, J=1.9 \mathrm{~Hz}, 1 \mathrm{H}), 8.67(\mathrm{~s}, 1 \mathrm{H}), 8.41(\mathrm{ddd}, J=8.2,2.2,1.0 \mathrm{~Hz}$, $1 \mathrm{H}), 8.31-8.28(\mathrm{~m}, 1 \mathrm{H}), 7.67(\mathrm{t}, J=8.0 \mathrm{~Hz}, 1 \mathrm{H}), 3.28(\mathrm{~s}, 2 \mathrm{H}), 1.43-1.41(\mathrm{~m}, 2 \mathrm{H})$, $1.16-1.13(\mathrm{~m}, 2 \mathrm{H}) .{ }^{13} \mathrm{C} \mathrm{NMR}\left(126 \mathrm{MHz}, \mathrm{CDCl}_{3}\right) \delta 199.8,195.0,148.5,138.1,133.7$, 130.0, 127.5, 123.1, 39.7, 28.9, 12.5. HRMS (ESI) Calcd. for $\mathrm{C}_{12} \mathrm{H}_{12} \mathrm{NO}_{4}\left(\mathrm{M}+\mathrm{H}^{+}\right) \mathrm{m} / \mathrm{z}$ 234.0760 , found 234.0762 .

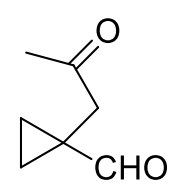

1-(2-oxopropyl)cyclopropanecarbaldehyde (4q): Purification by column chromatography $\left(\mathrm{SiO}_{2}\right.$, petroleum ether/ether $\left.=10: 1 \rightarrow 5: 1\right)$ gave an inseparable mixture of $\mathbf{4 q}$ and unreacted $\alpha$-hydroxycyclobutanone. Yield (determined by crude ${ }^{1} \mathrm{H}$ NMR) 60\%; yellow oil. IR (ATR): 3398, 3007, 2964, 2909, 1787, 1703, 1407, 1364, 1274, 1168, 1140, 1076, $915 \mathrm{~cm}^{-1} .{ }^{1} \mathrm{H}$ NMR $\left(500 \mathrm{MHz}, \mathrm{CDCl}_{3}\right) \delta 8.61(\mathrm{~s}, 1 \mathrm{H}), 2.66(\mathrm{~s}$, 2H), 2.19 (s, 3H), $1.32-1.29$ (m, 2H), $1.05-1.02$ (m, 2H). ${ }^{13} \mathrm{C}$ NMR (126 MHz, $\left.\mathrm{CDCl}_{3}\right) \delta$ 207.2, 206.0, 44.2, 30.1, 29.0, 12.6. HRMS (ESI) Calcd. for $\mathrm{C}_{7} \mathrm{H}_{11} \mathrm{O}_{2}\left(\mathrm{M}+\mathrm{H}^{+}\right)$ $\mathrm{m} / \mathrm{z} 127.0753$, found 127.0753 .

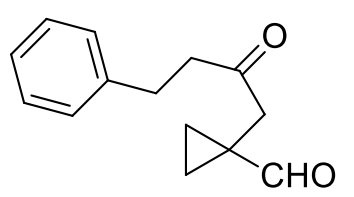

1-(2-oxo-4-phenylbutyl)cyclopropanecarbaldehyde (4r): Crude product was purified by flash column chromatography $\left(\mathrm{SiO}_{2}\right.$, petroleum ether/ether $\left.=10: 1 \rightarrow 1: 1\right)$. Yield 79\% (98 mg); colorless oil. IR (ATR): 3090, 3064, 3024, 2926, 1706, 1600, 1493, 1450, 1410, 1366, 1277, 1214, 1182, 1093, 1067, 1030, $981 \mathrm{~cm}^{-1} .{ }^{1} \mathrm{H}$ NMR (500 $\left.\mathrm{MHz}, \mathrm{CDCl}_{3}\right) \delta 8.60(\mathrm{~s}, 1 \mathrm{H}), 7.28-7.25(\mathrm{~m}, 2 \mathrm{H}), 7.19-7.17(\mathrm{~m}, 3 \mathrm{H}), 2.92-2.89(\mathrm{~m}$, $2 \mathrm{H}), 2.81-2.61(\mathrm{~m}, 2 \mathrm{H}), 2.61(\mathrm{~s}, 2 \mathrm{H}), 1.28-1.26(\mathrm{~m}, 2 \mathrm{H}), 1.00-0.98(\mathrm{~m}, 2 \mathrm{H}) .{ }^{13} \mathrm{C}$ NMR $\left(126 \mathrm{MHz}, \mathrm{CDCl}_{3}\right) \delta 207.1,200.1,141.1,128.5,128.4,126.2,44.4,43.5,29.7$, 28.9, 12.6. HRMS (ESI) Calcd. for $\mathrm{C}_{14} \mathrm{H}_{17} \mathrm{O}_{2}\left(\mathrm{M}+\mathrm{H}^{+}\right) \mathrm{m} / \mathrm{z}$ 217.1223, found 217.1223.

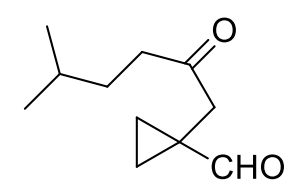


1-(5-methyl-2-oxohexyl)cyclopropanecarbaldehyde (4s): Crude product was purified by flash column chromatography $\left(\mathrm{SiO}_{2}\right.$, petroleum ether/ether $\left.=10: 1 \rightarrow 5: 1\right)$. Yield 54 \% (67 mg); colorless oil. IR (ATR): 3112, 3088, 3004, 2914, 2848, 2742, $1703,1687,1613,1580,1521,1480,1439,1344,1278,1262,1210,1082,1006,897$ $\mathrm{cm}^{-1} .{ }^{1} \mathrm{H}$ NMR $\left(500 \mathrm{MHz}, \mathrm{CDCl}_{3}\right) \delta 8.62(\mathrm{~s}, 1 \mathrm{H}), 2.65(\mathrm{~s}, 2 \mathrm{H}), 2.47-2.44(\mathrm{~m}, 2 \mathrm{H})$, $1.55-1.50(\mathrm{~m}, 1 \mathrm{H}), 1.49-1.45(\mathrm{~m}, 2 \mathrm{H}), 1.30-1.28(\mathrm{~m}, 2 \mathrm{H}), 1.03-1.01(\mathrm{~m}, 2 \mathrm{H})$, $0.88(\mathrm{~d}, J=6.5 \mathrm{~Hz}, 6 \mathrm{H}) .{ }^{13} \mathrm{C} \mathrm{NMR}\left(126 \mathrm{MHz}, \mathrm{CDCl}_{3}\right) \delta 208.3,200.2,43.2,40.9,32.5$, 28.9, 27.7, 22.4, 12.6. HRMS (ESI) Calcd. for $\mathrm{C}_{11} \mathrm{H}_{19} \mathrm{O}_{2}\left(\mathrm{M}+\mathrm{H}^{+}\right) \mathrm{m} / \mathrm{z} 183.1379$, found 183.1378 .

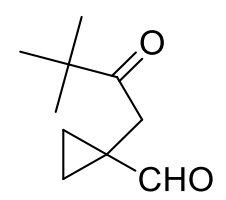

1-(3,3-dimethyl-2-oxobutyl)cyclopropanecarbaldehyde (4t): Crude product was purified by flash column chromatography $\left(\mathrm{SiO}_{2}\right.$, petroleum ether/ether $\left.=10: 1 \rightarrow 5: 1\right)$. Yield 72\% (70 mg); colorless oil. IR (ATR): 2969, 2912, 2874, 1703, 1476, 1366, 1323, 1073, $987 \mathrm{~cm}^{-1} .{ }^{1} \mathrm{H}$ NMR $\left(500 \mathrm{MHz}, \mathrm{CDCl}_{3}\right) \delta 8.66(\mathrm{~s}, 1 \mathrm{H}), 2.75(\mathrm{~s}, 2 \mathrm{H}), 1.29-$ $1.27(\mathrm{~m}, 2 \mathrm{H}), 1.16(\mathrm{~s}, 9 \mathrm{H}), 0.97-0.93(\mathrm{~m}, 2 \mathrm{H}) .{ }^{13} \mathrm{C} \mathrm{NMR}\left(126 \mathrm{MHz}, \mathrm{CDCl}_{3}\right) \delta 212.8$, 200.3, 44.0, 37.8, 28.5, 26.5, 12.7. HRMS (ESI) Calcd. for $\mathrm{C}_{10} \mathrm{H}_{17} \mathrm{O}_{2}\left(\mathrm{M}+\mathrm{H}^{+}\right) \mathrm{m} / \mathrm{z}$ 169.1223, found 169.1213 .

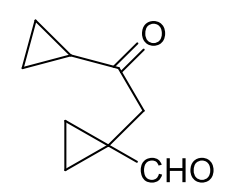

1-(2-cyclopropyl-2-oxoethyl)cyclopropanecarbaldehyde (4u): Crude product was purified by flash column chromatography $\left(\mathrm{SiO}_{2}\right.$, petroleum ether/ether $\left.=10: 1 \rightarrow 5: 1\right)$. Yield 68\% (60 mg); colorless oil. IR (ATR): 3039, 3010, 2903, 2831, 1752, 1700, $1447,1387,1277,1260,1197,1102,1082,1001 \mathrm{~cm}^{-1} .{ }^{1} \mathrm{H}$ NMR $\left(500 \mathrm{MHz}, \mathrm{CDCl}_{3}\right) \delta$ $8.66(\mathrm{~s}, 1 \mathrm{H}), 2.83(\mathrm{~s}, 2 \mathrm{H}), 1.98-1.95(\mathrm{~m}, 1 \mathrm{H}), 1.31-1.28(\mathrm{~m}, 2 \mathrm{H}), 1.05-1.02(\mathrm{~m}$, 4H), $0.90-0.86(\mathrm{~m}, 2 \mathrm{H}) .{ }^{13} \mathrm{C} \mathrm{NMR}\left(126 \mathrm{MHz}, \mathrm{CDCl}_{3}\right) \delta 207.9,200.2,43.9,28.7,20.6$, 12.9, 11.0. HRMS (ESI) Calcd. for $\mathrm{C}_{9} \mathrm{H}_{13} \mathrm{O}_{2}\left(\mathrm{M}+\mathrm{H}^{+}\right) \mathrm{m} / \mathrm{z}$ 153.0910, found 153.0909. 


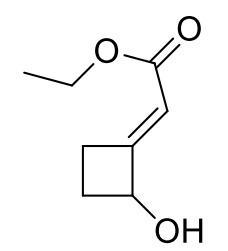

(E)-ethyl 2-(2-hydroxycyclobutylidene)acetate (3w): Crude product was purified by flash column chromatography $\left(\mathrm{SiO}_{2}\right.$, petroleum ether/ether $\left.=5: 1 \rightarrow 1: 1\right)$. Major isomer: yield 60\% (54 mg); colorless oil. IR (ATR): 3412, 2985, 2952, 2908, 1787, 1700, 1449, 1425, 1395, 1371, 1273, 1229, 1187, 1167, 1137, 1074, $936 \mathrm{~cm}^{-1} .{ }^{1} \mathrm{H}$ NMR $(500 \mathrm{MHz}$, $\left.\mathrm{CDCl}_{3}\right) \delta 5.81-5.79(\mathrm{~m}, 1 \mathrm{H}), 4.74-4.70(\mathrm{~m}, 1 \mathrm{H}), 4.15-4.11(\mathrm{~m}, 2 \mathrm{H}), 3.28(\mathrm{br} \mathrm{s}$, $1 \mathrm{H}), 2.96-2.93(\mathrm{~m}, 1 \mathrm{H}), 2.55-2.48(\mathrm{~m}, 1 \mathrm{H}), 2.42-2.38(\mathrm{~m}, 1 \mathrm{H}), 1.92-1.87(\mathrm{~m}$, $1 \mathrm{H}), 1.24(\mathrm{t}, J=7.1 \mathrm{~Hz}, 3 \mathrm{H}) .{ }^{13} \mathrm{C} \mathrm{NMR}\left(126 \mathrm{MHz}, \mathrm{CDCl}_{3}\right) \delta 168.4,166.8,110.7,71.3$, 60.1, 29.7, 25.3, 14.3. HRMS (ESI) Calcd. for $\mathrm{C}_{8} \mathrm{H}_{13} \mathrm{O}_{3}\left(\mathrm{M}+\mathrm{H}^{+}\right) \mathrm{m} / \mathrm{z}$ 157.0859, found 157.0859 .

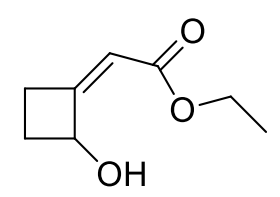

(Z)-ethyl 2-(2-hydroxycyclobutylidene)acetate (3w): Minor isomer: yield 10\% (9 mg); colorless oil. IR (ATR): 3431, 2990, 2957, 2911, 1692, 1670, 1469, 1447, 1393 , 1374, 1338, 1238, 1210, 1129, 1085, 1039, $911 \mathrm{~cm}^{-1} .{ }^{1} \mathrm{H}$ NMR (500 MHz, $\left.\mathrm{CDCl}_{3}\right) \delta$ $5.68-5.67(\mathrm{~m}, 1 \mathrm{H}), 5.05$ (br s, $2 \mathrm{H}), 4.21-4.17(\mathrm{~m}, 2 \mathrm{H}), 2.57-2.49(\mathrm{~m}, 2 \mathrm{H}), 2.38-$ $2.36(\mathrm{~m}, 1 \mathrm{H}), 2.08-2.03(\mathrm{~m}, 1 \mathrm{H}), 1.29(\mathrm{t}, J=7.2 \mathrm{~Hz}, 3 \mathrm{H}) .{ }^{13} \mathrm{C}$ NMR $(126 \mathrm{MHz}$, $\left.\mathrm{CDCl}_{3}\right) \delta 169.8,167.9,113.2,71.0,60.8,25.9,24.1,14.3$. HRMS (ESI) Calcd. for $\mathrm{C}_{8} \mathrm{H}_{13} \mathrm{O}_{3}\left(\mathrm{M}+\mathrm{H}^{+}\right) \mathrm{m} / \mathrm{z}$ 157.0859, found 157.0860.

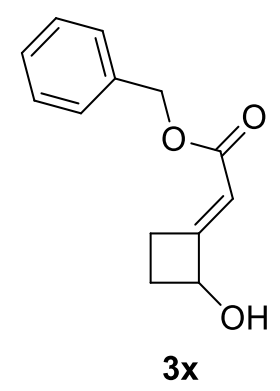

(E)-benzyl 2-(2-hydroxycyclobutylidene)acetate (3x): Crude product was purified by flash column chromatography $\left(\mathrm{SiO}_{2}\right.$, petroleum ether/ether $\left.=5: 1 \rightarrow 1: 1\right)$. Major isomer: yield 34\% (52 mg); yellow oil. IR (ATR): 3427, 3070, 3035, 2992, 2955, 1692, 12 
$1669,1502,1456,1381,1343,1202,1188,1125,1084,1001,860 \mathrm{~cm}^{-1} .{ }^{1} \mathrm{H}$ NMR $(500$ $\left.\mathrm{MHz}, \mathrm{CDCl}_{3}\right) \delta 7.36-7.30(\mathrm{~m}, 5 \mathrm{H}), 5.91(\mathrm{dd}, J=4.5,2.2 \mathrm{~Hz}, 1 \mathrm{H}), 5.15(\mathrm{~s}, 2 \mathrm{H}), 4.75$ (t, $J=7.5 \mathrm{~Hz}, 1 \mathrm{H}), 3.04-2.97(\mathrm{~m}, 1 \mathrm{H}), 2.68($ br s, $1 \mathrm{H}), 2.57-2.51(\mathrm{~m}, 1 \mathrm{H}), 2.45-$ $2.39(\mathrm{~m}, 1 \mathrm{H}), 1.94-1.87(\mathrm{~m}, 1 \mathrm{H}) .{ }^{13} \mathrm{C} \mathrm{NMR}\left(126 \mathrm{MHz}, \mathrm{CDCl}_{3}\right) \delta 168.9,166.3,136.2$, 128.6, 128.23, 128.22, 110.5, 71.4, 66.0, 29.9, 25.4. HRMS (ESI) Calcd. for $\mathrm{C}_{13} \mathrm{H}_{15} \mathrm{O}_{3}$ $\left(\mathrm{M}+\mathrm{H}^{+}\right) \mathrm{m} / \mathrm{z} 219.1015$, found 219.1017.

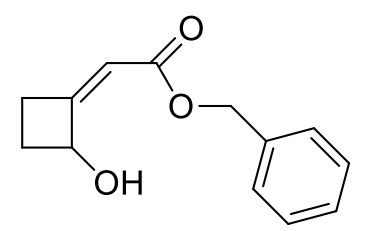

(Z)-benzyl 2-(2-hydroxycyclobutylidene)acetate (3x): Minor isomer: yield 12\% (18 mg); yellow oil. IR (ATR): 3410, 3070, 3036, 2992, 2952, 1703, 1502, 1421, 1381, 1338, 1271, 1188, 1162, 1021, $909 \mathrm{~cm}^{-1} .{ }^{1} \mathrm{H}$ NMR (500 MHz, $\left.\mathrm{CDCl}_{3}\right) \delta 7.37$ - $7.32(\mathrm{~m}$, $5 \mathrm{H}), 5.75-5.74(\mathrm{~m}, 1 \mathrm{H}), 5.17(\mathrm{~s}, 2 \mathrm{H}), 5.09-5.03(\mathrm{~m}, 1 \mathrm{H}), 2.58-2.48(\mathrm{~m}, 2 \mathrm{H}), 2.39$ - $2.36(\mathrm{~m}, 1 \mathrm{H}), 2.10-2.02(\mathrm{~m}, 1 \mathrm{H}) .{ }^{13} \mathrm{C} \mathrm{NMR}\left(126 \mathrm{MHz}, \mathrm{CDCl}_{3}\right) \delta$ 170.6, 167.5, 135.7, 128.7, 128.4, 128.3, 113.0, 71.1, 66.5, 25.9, 24.2. HRMS (ESI) Calcd. for $\mathrm{C}_{13} \mathrm{H}_{15} \mathrm{O}_{3}\left(\mathrm{M}+\mathrm{H}^{+}\right) \mathrm{m} / \mathrm{z} 219.1015$, found 219.0991.

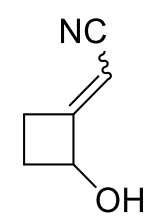

2-(2-hydroxycyclobutylidene)acetonitrile (3y): Crude product was purified by flash column chromatography $\left(\mathrm{SiO}_{2}\right.$, petroleum ether/ether $\left.=5: 1 \rightarrow 1: 1\right)$. Yield 58\% (37 mg); yellow oil. IR (ATR): 3385, 3058, 3004, 2955, 2223, 1670, 1423, 1317, 1276, 1224, $1183,1131,1085,979 \mathrm{~cm}^{-1}$. NMR data were obtained from a not separable d.r. 78:22 mixture: ${ }^{1} \mathrm{H}$ NMR (500 MHz, $\left.\mathrm{CDCl}_{3}\right) \delta 5.41-5.40(\mathrm{~m}, 1 \mathrm{H}), 5.20-5.18(\mathrm{~m}, 1 \mathrm{H}), 4.97$ - $4.94(\mathrm{~m}, 1 \mathrm{H}), 4.79-4.75(\mathrm{~m}, 1 \mathrm{H}), 2.76-2.68(\mathrm{~m}, 3 \mathrm{H}), 2.59-2.57(\mathrm{~m}, 1 \mathrm{H}), 2.53-$ $2.43(\mathrm{~m}, 4 \mathrm{H}), 2.06-1.95(\mathrm{~m}, 1 \mathrm{H}), 1.93-1.89(\mathrm{~m}, 1 \mathrm{H}) .{ }^{13} \mathrm{C} \mathrm{NMR}\left(126 \mathrm{MHz}, \mathrm{CDCl}_{3}\right)$ $\delta 173.4,173.0,115.9,115.8,90.5,90.1,71.4,71.2,28.9,28.1,25.2,24.4$. HRMS (ESI) Calcd. for $\mathrm{C}_{6} \mathrm{H}_{8} \mathrm{NO}\left(\mathrm{M}+\mathrm{H}^{+}\right) \mathrm{m} / \mathrm{z} 110.0600$, found 110.0600 . 
Procedure for the preparation of (E)-2-(2-hydroxycyclobutylidene)-1phenylethanone (3a) (Table 1, entry 4)<smiles>O=C(/C=C1\CCCO1)c1ccccc1</smiles>

A solution of $1(0.05 \mathrm{~g}, 0.58 \mathrm{mmol})$ and phosphonium ylide $\mathbf{2 a}(0.220 \mathrm{~g}, 0.58 \mathrm{mmol})$ in $\mathrm{CH}_{2} \mathrm{Cl}_{2}(0.5 \mathrm{~mL})$ was stirred at reflux for $48 \mathrm{~h}$. The crude mixture, without aqueous work-up, was directly purified by flash column chromatography $\left(\mathrm{SiO}_{2}\right.$, petroleum ether/ether $=5: 1 \rightarrow 1: 1)$ to give an inseparable mixture of $\mathbf{3 a}$ and triphenylphosphine oxide. Yield (determined by crude ${ }^{1} \mathrm{H}$ NMR) 66\%; colorless oil. IR (ATR): 3390, 3066, 2998, 2952, 1687, 1613, 1600, 1450, 1368, 1246, 1219, 1129, $908 \mathrm{~cm}^{-1}$. ${ }^{1} \mathrm{H}$ NMR (500 $\left.\mathrm{MHz}, \mathrm{CDCl}_{3}\right) \delta 7.92-7.90(\mathrm{~m}, 2 \mathrm{H}), 7.53-7.50(\mathrm{~m}, 1 \mathrm{H}), 7.44-7.40(\mathrm{~m}, 2 \mathrm{H}), 7.00-$ $6.99(\mathrm{~m}, 1 \mathrm{H}), 4.89-4.84(\mathrm{~m}, 1 \mathrm{H}), 3.04-3.00(\mathrm{~m}, 1 \mathrm{H}), 2.67-2.63(\mathrm{~m}, 1 \mathrm{H}), 2.48-$ $2.45(\mathrm{~m}, 1 \mathrm{H}), 2.01-1.94(\mathrm{~m}, 1 \mathrm{H}) .{ }^{13} \mathrm{C} \mathrm{NMR}\left(126 \mathrm{MHz}, \mathrm{CDCl}_{3}\right) \delta 190.8,169.7,138.6$, 132.7, 128.6, 128.3 , 114.1, 71.9, 30.5, 26.3. HRMS (ESI) Calcd. for $\mathrm{C}_{12} \mathrm{H}_{13} \mathrm{O}_{2}\left(\mathrm{M}+\mathrm{H}^{+}\right)$ $\mathrm{m} / \mathrm{z} 189.0910$, found 189.0910 .

\section{General procedure for the preparation of cyclobutylidenes $6 \mathrm{w}-\mathrm{y}$.}

To a solution of $\mathbf{3 w}-\mathbf{y}(1.75 \mathrm{mmol})$ in DMF (4 mL) were added imidazole (0.143 g, 2.1 $\mathrm{mmol})$ and tert-butylchlorodimethylsilane $(2.1 \mathrm{mmol})$. The resulting mixture was stirred $24 \mathrm{~h}$ at room temperature. Water was added and the aqueous layer was extracted with diethyl ether. The combinated organic layers were dried and evaporated under reduced pressure. The residue was purified by flash column chromatography (see below for details) to give compound $\mathbf{6 w - y}$.

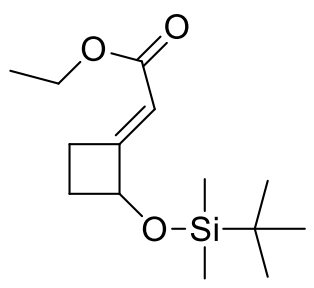

(E)-ethyl 2-(2-((tert-butyldimethylsilyl)oxy)cyclobutylidene)acetate (6w): Crude product was purified by flash column chromatography $\left(\mathrm{SiO}_{2}\right.$, petroleum ether/ether $=$ 
10:1 $\rightarrow 5: 1$ ). Yield $89 \%$ (420 mg); yellow oil. IR (ATR): 2985, 2952, 2930, 2859, 1719, 1472, 1365, 1327, 1254, 1189, 1153, 1080, 1044, $949 \mathrm{~cm}^{-1}$. ${ }^{1} \mathrm{H}$ NMR (500 MHz, $\left.\mathrm{CDCl}_{3}\right) \delta 5.77(\mathrm{~d}, J=2.3 \mathrm{~Hz}, 1 \mathrm{H}), 4.77-4.72(\mathrm{~m}, 1 \mathrm{H}), 4.18-4.13(\mathrm{~m}, 2 \mathrm{H}), 3.01-$ $2.96(\mathrm{~m}, 1 \mathrm{H}), 2.55-2.47(\mathrm{~m}, 1 \mathrm{H}), 2.35-2.28(\mathrm{~m}, 1 \mathrm{H}), 1.98-1.92(\mathrm{~m}, 1 \mathrm{H}), 1.27(\mathrm{t}, J$ $=7.1 \mathrm{~Hz}, 3 \mathrm{H}), 0.90(\mathrm{~s}, 9 \mathrm{H}), 0.09(\mathrm{~s}, 3 \mathrm{H}), 0.08(\mathrm{~s}, 3 \mathrm{H}) \cdot{ }^{13} \mathrm{C} \mathrm{NMR}\left(126 \mathrm{MHz}, \mathrm{CDCl}_{3}\right) \delta$ $168.1,166.6,110.3,71.3,59.8,29.9,25.8,25.3,18.2,14.4,-4.6,-4.7 . \mathrm{MS}: \mathrm{m} / \mathrm{z}(\%)=$ 270 (45) $\left[\mathrm{M}^{+}\right], 241$ (14), 213 (29), 185 (18), 103 (100), 73 (100).

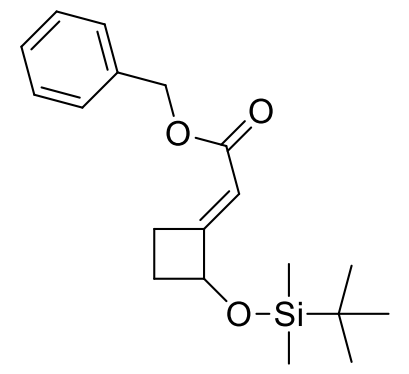

(E)-benzyl 2-(2-((tert-butyldimethylsilyl)oxy)cyclobutylidene)acetate (6x): Crude product was purified by flash column chromatography $\left(\mathrm{SiO}_{2}\right.$, petroleum ether/ether $=$ 10:1 $\rightarrow 5: 1$ ). Yield $86 \%$ (499 mg); yellow oil. IR (ATR): 3067, 3035, 2958, 2929, 2892, 1720, 1686, 1470, 1461, 1326, 1260, 1159, 1101, $952 \mathrm{~cm}^{-1} .{ }^{1} \mathrm{H}$ NMR $(500 \mathrm{MHz}$, $\left.\mathrm{CDCl}_{3}\right) \delta 7.37-7.29(\mathrm{~m}, 5 \mathrm{H}), 5.83(\mathrm{~d}, J=2.1 \mathrm{~Hz}, 1 \mathrm{H}), 5.18-5.12(\mathrm{~m}, 2 \mathrm{H}), 4.77-$ $4.74(\mathrm{~m}, 1 \mathrm{H}), 3.03-2.96(\mathrm{~m}, 1 \mathrm{H}), 2.55-2.48(\mathrm{~m}, 1 \mathrm{H}), 2.35-2.28(\mathrm{~m}, 1 \mathrm{H}), 1.98-$ $1.90(\mathrm{~m}, 1 \mathrm{H}), 0.90(\mathrm{~s}, 9 \mathrm{H}), 0.09(\mathrm{~s}, 3 \mathrm{H}), 0.07(\mathrm{~s}, 3 \mathrm{H}) .{ }^{13} \mathrm{C} \mathrm{NMR}\left(126 \mathrm{MHz}, \mathrm{CDCl}_{3}\right) \delta$ 169.0, 166.4, 136.4, 128.6, 128.3, 128.2, 110.0, 71.3, 65.8, 29.9, 25.9, 25.4, 18.2, -4.6, -4.7. MS: $m / z(\%)=275(3)\left[\mathrm{M}^{+}-57\right], 241(36), 165(7), 135$ (9), 91 (100).

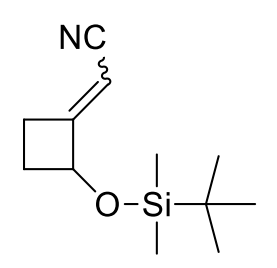

2-(2-((tert-butyldimethylsilyl)oxy)cyclobutylidene)acetonitrile (6y): Crude product was purified by flash column chromatography $\left(\mathrm{SiO}_{2}\right.$, petroleum ether/ether = 10:1 $\rightarrow 5: 1$ ). Yield $92 \%$ (358 mg); yellow oil. IR (ATR): 2952, 2929, 2889, 2221, 1671, $1473,1424,1364,1254,1153,1090,946 \mathrm{~cm}^{-1}$. NMR data were obtained from a not separable d.r. 81:19 mixture. Major diastereoisomer: ${ }^{1} \mathrm{H}$ NMR $\left(500 \mathrm{MHz}, \mathrm{CDCl}_{3}\right) \delta$ 
$5.28(\mathrm{~d}, J=2.4 \mathrm{~Hz}, 1 \mathrm{H}), 4.75-4.71(\mathrm{~m}, 1 \mathrm{H}), 2.69-2.66(\mathrm{~m}, 1 \mathrm{H}), 2.45-2.40(\mathrm{~m}, 1 \mathrm{H})$, $2.37-2.32(\mathrm{~m}, 1 \mathrm{H}), 1.93-1.89(\mathrm{~m}, 1 \mathrm{H}), 0.89(\mathrm{~s}, 9 \mathrm{H}), 0.08(\mathrm{~s}, 3 \mathrm{H}), 0.07(\mathrm{~s}, 3 \mathrm{H}) .{ }^{13} \mathrm{C}$ NMR $\left(126 \mathrm{MHz}, \mathrm{CDCl}_{3}\right) \delta 172.7,116.1,89.4,71.3,29.0,25.7,24.4,18.1,-4.6,-4.7$. MS: $m / z(\%)=223(8)\left[\mathrm{M}^{+}\right], 166(100), 136(28), 102(40), 73(44)$.

General procedure for the preparation of cyclopropanecarbaldehydes $4 \mathrm{w}-\mathrm{y}$. To a solution of $\mathbf{6 w}-\mathbf{y}(0.27 \mathrm{mmol})$ in 1,4-dioxane $(0.25 \mathrm{~mL}) / \mathrm{H}_{2} \mathrm{O}(0.25 \mathrm{~mL})$ was added tetrabutylammonium fluoride $(0.103 \mathrm{~g}, 0.33 \mathrm{mmol})$ and the mixture was stirred at reflux for $48 \mathrm{~h}$. The reaction mixture was then partitioned between brine and EtOAc. The layers were separated and the aqueous phase was extracted with EtOAc. The combined organic extracts were washed with brine, dried over anhydrous $\mathrm{Na}_{2} \mathrm{SO}_{4}$, filtered, and concentrated under reduced pressure. The crude product was purified by column chromatography on silica gel to give cyclopropanecarbaldehyde $\mathbf{4 w}-\mathbf{y}$.

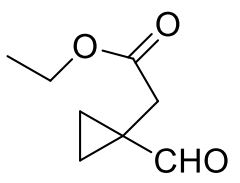

Ethyl 2-(1-formylcyclopropyl)acetate (4w): Crude product was purified by flash column chromatography $\left(\mathrm{SiO}_{2}\right.$, petroleum ether/ether $\left.=10: 1 \rightarrow 5: 1\right)$. Yield 74\% (31 mg); colorless oil. IR (ATR): 2987, 2923, 2854, 1732, 1709, 1467, 1375, 1329, 1277, 1260, 1182, 1033, $909 \mathrm{~cm}^{-1} .{ }^{1} \mathrm{H}$ NMR (500 MHz, $\left.\mathrm{CDCl}_{3}\right) \delta 8.73(\mathrm{~s}, 1 \mathrm{H}), 4.15$ (q, $J=$ $7.2 \mathrm{~Hz}, 2 \mathrm{H}), 2.59$ (s, 2H), $1.30(\mathrm{q}, J=4.8 \mathrm{~Hz}, 2 \mathrm{H}), 1.25(\mathrm{t}, J=7.1 \mathrm{~Hz}, 3 \mathrm{H}), 1.08(\mathrm{q}, J$ $=4.8 \mathrm{~Hz}, 2 \mathrm{H}) .{ }^{13} \mathrm{C} \mathrm{NMR}\left(126 \mathrm{MHz}, \mathrm{CDCl}_{3}\right) \delta 200.1,171.2,60.8,35.5,29.0,14.3,13.1$. HRMS (ESI) Calcd. for $\mathrm{C}_{8} \mathrm{H}_{13} \mathrm{O}_{3}\left(\mathrm{M}+\mathrm{H}^{+}\right) \mathrm{m} / \mathrm{z}$ 157.0859, found 157.0859.

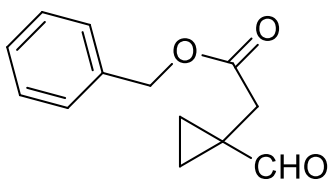

Benzyl 2-(1-formylcyclopropyl)acetate $(\mathbf{4 x})$ : Crude product was purified by flash column chromatography $\left(\mathrm{SiO}_{2}\right.$, petroleum ether/ether $\left.=10: 1 \rightarrow 5: 1\right)$. Yield $70 \%(41$ mg); yellow oil. IR (ATR): 3064, 3035, 3010, 2961, 2920, 1735, 1703, 1455, 1301, 1284, 1254, 1159, 1067, 1012, $903 \mathrm{~cm}^{-1} .{ }^{1} \mathrm{H}$ NMR (500 MHz, $\left.\mathrm{CDCl}_{3}\right) \delta 8.72(\mathrm{~s}, 1 \mathrm{H})$, $7.38-7.30(\mathrm{~m}, 5 \mathrm{H}), 5.14(\mathrm{~s}, 2 \mathrm{H}), 2.65(\mathrm{~s}, 2 \mathrm{H}), 1.32-1.30(\mathrm{~m}, 2 \mathrm{H}), 1.11-1.07(\mathrm{~m}$, 
2H). ${ }^{13} \mathrm{C}$ NMR (126 MHz, $\left.\mathrm{CDCl}_{3}\right) \delta 200.0,171.0,135.9,128.6,128.3,128.2,66.6$, 35.5, 29.0, 13.0. HRMS (ESI) Calcd. for $\mathrm{C}_{13} \mathrm{H}_{15} \mathrm{O}_{3}\left(\mathrm{M}+\mathrm{H}^{+}\right) \mathrm{m} / \mathrm{z}$ 219.1015, found 219.1015 .

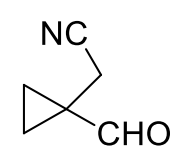

2-(1-formylcyclopropyl)acetonitrile (4y): Crude product was purified by flash column chromatography $\left(\mathrm{SiO}_{2}\right.$, petroleum ether/ether $\left.=10: 1 \rightarrow 5: 1\right)$. Yield 62\% $(18$ mg); yellow oil. IR (ATR): 3010, 2932, 2828, 2253, 1712, 1415, 1366, 1277, 1260, 1225, 1073, 1038, $1007 \mathrm{~cm}^{-1} .{ }^{1} \mathrm{H}$ NMR $\left(500 \mathrm{MHz}, \mathrm{CDCl}_{3}\right) \delta 8.62(\mathrm{~s}, 1 \mathrm{H}), 2.80(\mathrm{~s}, 2 \mathrm{H})$, $1.38-1.36(\mathrm{~m}, 2 \mathrm{H}), 1.31-1.29(\mathrm{~m}, 2 \mathrm{H}) .{ }^{13} \mathrm{C} \mathrm{NMR}\left(126 \mathrm{MHz}, \mathrm{CDCl}_{3}\right) \delta 197.8,116.9$, 28.5, 18.4, 12.1. HRMS (ESI) Calcd. for $\mathrm{C}_{6} \mathrm{H}_{8} \mathrm{NO}\left(\mathrm{M}+\mathrm{H}^{+}\right) \mathrm{m} / \mathrm{z} 110.0600$, found 110.0608 .

\section{Procedure for the preparation of 2-(1-formylcyclopropyl)acetic acid (4z).}

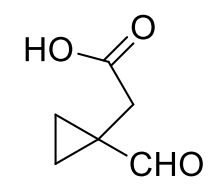

To a solution of $\mathbf{6 w}(1.18 \mathrm{mmol}, 319 \mathrm{mg})$ in 1,4-dioxane $(2.4 \mathrm{~mL}) / \mathrm{H}_{2} \mathrm{O}(1.1 \mathrm{~mL})$ was added sodium hydroxide $(4.72 \mathrm{mmol}, 189 \mathrm{mg})$ and the mixture was stirred at reflux for $72 \mathrm{~h}$. After the mixture was cooled to $21^{\circ} \mathrm{C}$, water was added, and the mixture was extracted with ethyl acetate. The aqueous phase was acidified with $1.0 \mathrm{M}$ aqueous $\mathrm{HCl}$ to $\mathrm{pH} 1$. The acidified aqueous layer was extracted with ethyl acetate, and the combined organic phases were washed with water and brine and dried over anhydrous $\mathrm{Na}_{2} \mathrm{SO}_{4}$. Flash column chromatography on silica gel, eluting with 1/1 petroleum ether/ethyl acetate, gave the cyclopropanecarbaldehyde 4z: Yield 67\% (23 mg); yellow oil. IR (ATR): 3082, 3012, 2933, 1700, 1692, 1434, 1385, 1365, 1336, 1287, 1227, 1014, 941, 911, $900 \mathrm{~cm}^{-1} .{ }^{1} \mathrm{H}$ NMR $\left(500 \mathrm{MHz}, \mathrm{CDCl}_{3}\right) \delta 10.07$ (br s, $\left.1 \mathrm{H}\right), 8.66(\mathrm{~s}, 1 \mathrm{H}), 2.59$ (s, $2 \mathrm{H}), 1.32-1.29(\mathrm{~m}, 2 \mathrm{H}), 1.10-1.07(\mathrm{~m}, 2 \mathrm{H}) .{ }^{13} \mathrm{C} \mathrm{NMR}\left(126 \mathrm{MHz}, \mathrm{CDCl}_{3}\right) \delta 200.4$, 177.0, 35.2, 28.8, 13.1. HRMS (ESI) Calcd. for $\mathrm{C}_{6} \mathrm{H}_{9} \mathrm{O}_{3}\left(\mathrm{M}+\mathrm{H}^{+}\right) \mathrm{m} / \mathrm{z}$ 129.0546, found 129.0546 .

Procedure for the preparation of cyclopropylketone 4aa and 1,4-dione 7. 
A solution of 1' $(0.066 \mathrm{~g}, 0.58 \mathrm{mmol})$ and phosphonium ylide $2 \mathbf{2 a}(0.220 \mathrm{~g}, 0.58 \mathrm{mmol})$ in toluene $(0.5 \mathrm{~mL})$ was stirred in a sealed tube reactor at $110{ }^{\circ} \mathrm{C}$ for 5 days. The crude mixture, without aqueous work-up, was directly purified by flash column chromatography $\left(\mathrm{SiO}_{2}\right.$, petroleum ether/ether $\left.=10: 1 \rightarrow 5: 1\right)$ to give compound $\mathbf{4 a a}$ and 7.<smiles>CCC(=O)C1(CC(=O)c2ccccc2)CC1</smiles>

1-(1-(2-oxo-2-phenylethyl)cyclopropyl)propan-1-one (4aa): yield 41\% (51 mg); orange oil. IR (ATR): 3083, 3060, 2979, 2936, 2905, 1687, 1598, 1579, 1451, 1409, 1378, 1355, 1215, $1103983 \mathrm{~cm}^{-1} .{ }^{1} \mathrm{H}$ NMR $\left(500 \mathrm{MHz}, \mathrm{CDCl}_{3}\right) \delta 7.96-7.95$ (m, 2H), 7.55 (dd, $J=10.5,4.3 \mathrm{~Hz}, 1 \mathrm{H}), 7.45$ (dd, $J=10.6,4.8 \mathrm{~Hz}, 2 \mathrm{H}), 3.28$ (s, 2H), 2.25 (q, $J=7.3 \mathrm{~Hz}, 2 \mathrm{H}), 1.40(\mathrm{q}, J=4.9 \mathrm{~Hz}, 2 \mathrm{H}), 1.03(\mathrm{t}, J=7.3 \mathrm{~Hz}, 3 \mathrm{H}), 0.89$ (q, $J=4.9 \mathrm{~Hz}$, 2H). ${ }^{13} \mathrm{C} \mathrm{NMR}\left(126 \mathrm{MHz}, \mathrm{CDCl}_{3}\right) \delta 209.9,198.0,137.0,133.1,128.7,128.1,43.3$, 29.0, 28.1, 15.1, 8.3. HRMS (ESI) Calcd. for $\mathrm{C}_{14} \mathrm{H}_{17} \mathrm{O}_{2}\left(\mathrm{M}+\mathrm{H}^{+}\right) \mathrm{m} / \mathrm{z} 217.1223$, found 217.1225.

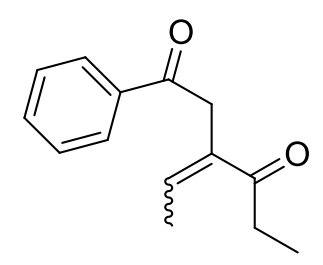

3-ethylidene-1-phenylhexane-1,4-dione (7): yield 20\% (25 mg); yellow oil. IR (ATR): 3068, 2979, 2936, 2917, 2874, 1687, 1668, 1598, 1451, 1389, 1331, 1250, $1212,1181,1018 \mathrm{~cm}^{-1} .{ }^{1} \mathrm{H}$ NMR $\left(500 \mathrm{MHz}, \mathrm{CDCl}_{3}\right) \delta 8.02-7.98(\mathrm{~m}, 2 \mathrm{H}), 7.58-7.53$ (m, 1H), $7.46(\mathrm{t}, J=7.6 \mathrm{~Hz}, 2 \mathrm{H}), 7.03(\mathrm{q}, J=6.9 \mathrm{~Hz}, 1 \mathrm{H}), 4.02$ (s, 2H), 2.75 (q, $J=7.3$ $\mathrm{Hz}, 2 \mathrm{H}), 1.87(\mathrm{~d}, J=7.0 \mathrm{~Hz}, 3 \mathrm{H}), 1.10(\mathrm{t}, J=7.3 \mathrm{~Hz}, 3 \mathrm{H}) .{ }^{13} \mathrm{C} \mathrm{NMR}\left(126 \mathrm{MHz}, \mathrm{CDCl}_{3}\right)$ $\delta$ 201.2 197.0, 140.2 137.1, 137.0, 133.1, 128.6, 128.4, 35.5, 30.1, 15.3, 8.7. Calcd. for $\mathrm{C}_{14} \mathrm{H}_{17} \mathrm{O}_{2}\left(\mathrm{M}+\mathrm{H}^{+}\right) \mathrm{m} / \mathrm{z} 217.1223$, found 217.1221.

General procedure for the preparation of cyclopropylenones 5. 
A solution of $1(0.58 \mathrm{mmol})$ and phosphonium ylide $2(1.16 \mathrm{mmol})$ in toluene $(0.5 \mathrm{~mL})$ was stirred in a sealed tube reactor at $110{ }^{\circ} \mathrm{C}$ for $96 \mathrm{~h}$. The crude mixture, without aqueous work-up, was directly purified by flash column chromatography (see below for details) to give compound $\mathbf{5}$.

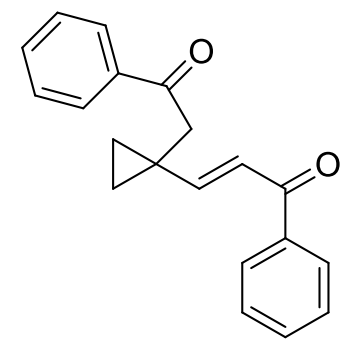

(E)-3-(1-(2-oxo-2-phenylethyl)cyclopropyl)-1-phenylprop-2-en-1-one (5a): Crude product was purified by flash column chromatography $\left(\mathrm{SiO}_{2}\right.$, petroleum ether/ether $=$ 10:1 $\rightarrow 5: 1$ ). Yield 70\% (117 mg); white solid; m. p. $=107-109{ }^{\circ} \mathrm{C}$. IR (ATR): 3096, 3006, 2965, 2938, 2906, 1681, 1659, 1597, 1572, 1510, 1458, 1417, 1352, 1308, 1251, 1213, 1167, 1023, 982, $938 \mathrm{~cm}^{-1} .{ }^{1} \mathrm{H}$ NMR $\left(500 \mathrm{MHz}, \mathrm{CDCl}_{3}\right) \delta 7.98(\mathrm{~d}, J=8.2 \mathrm{~Hz}$, 2H), $7.78(\mathrm{~d}, J=8.2 \mathrm{~Hz}, 2 \mathrm{H}), 7.62-7.58(\mathrm{~m}, 1 \mathrm{H}), 7.51-7.47(\mathrm{~m}, 3 \mathrm{H}), 7.38(\mathrm{t}, J=7.6$ $\mathrm{Hz}, 2 \mathrm{H}), 6.74(\mathrm{~d}, J=15.5 \mathrm{~Hz}, 1 \mathrm{H}), 6.63(\mathrm{~d}, J=15.5 \mathrm{~Hz}, 1 \mathrm{H}), 3.29(\mathrm{~s}, 2 \mathrm{H}), 1.13(\mathrm{t}, J=$ $5.8 \mathrm{~Hz}, 2 \mathrm{H}), 1.05(\mathrm{t}, J=5.8 \mathrm{~Hz}, 2 \mathrm{H}) .{ }^{13} \mathrm{C} \mathrm{NMR}\left(126 \mathrm{MHz}, \mathrm{CDCl}_{3}\right) \delta 197.5,190.3$, 156.1, 138.3, 137.2, 133.4, 132.4, 128.8, 128.53, 128.51, 128.1, 43.6, 20.1, 16.9 . HRMS (ESI) Calcd. for $\mathrm{C}_{20} \mathrm{H}_{19} \mathrm{O}_{2}\left(\mathrm{M}+\mathrm{H}^{+}\right) \mathrm{m} / \mathrm{z} 291.1379$, found 291.1379.

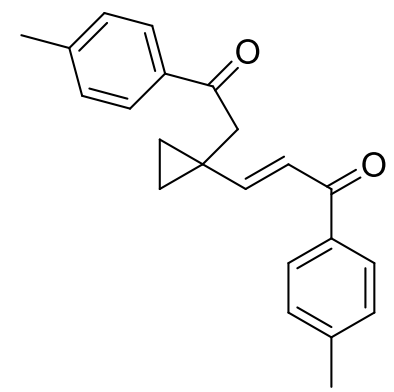

\section{(E)-3-(1-(2-oxo-2-(p-tolyl)ethyl)cyclopropyl)-1-(p-tolyl)prop-2-en-1-one}

(5b):

Crude product was purified by flash column chromatography $\left(\mathrm{SiO}_{2}\right.$, petroleum ether/ether $=10: 1 \rightarrow 5: 1)$. Yield 73\% (134 mg); yellow semi-solid. IR (ATR): 3061, 3034, 3004, 2922, 1684, 1662, 1608, 1572, 1450, 1409, 1355, 1259, 1224, 1205, 1183, 1039, $938 \mathrm{~cm}^{-1} .{ }^{1} \mathrm{H}$ NMR $\left(500 \mathrm{MHz}, \mathrm{CDCl}_{3}\right) \delta 7.89(\mathrm{~d}, J=8.1 \mathrm{~Hz}, 2 \mathrm{H}), 7.70(\mathrm{~d}, J=$ $8.1 \mathrm{~Hz}, 2 \mathrm{H}), 7.29(\mathrm{~d}, J=7.9 \mathrm{~Hz}, 2 \mathrm{H}), 7.18(\mathrm{~d}, J=7.8 \mathrm{~Hz}, 2 \mathrm{H}), 6.72(\mathrm{~d}, J=15.5 \mathrm{~Hz}$, 
$1 \mathrm{H}), 6.63(\mathrm{~d}, J=15.5 \mathrm{~Hz}, 1 \mathrm{H}), 3.26(\mathrm{~s}, 2 \mathrm{H}), 2.44(\mathrm{~s}, 3 \mathrm{H}), 2.37(\mathrm{~s}, 3 \mathrm{H}), 1.12(\mathrm{t}, J=5.5$ $\mathrm{Hz}, 2 \mathrm{H}), 1.03(\mathrm{t}, J=5.6 \mathrm{~Hz}, 2 \mathrm{H}) .{ }^{13} \mathrm{C} \mathrm{NMR}\left(126 \mathrm{MHz}, \mathrm{CDCl}_{3}\right) \delta 197.2,189.8,155.7$, 144.2, 143.1, 135.7, 134.8, 129.5, 129.2, 128.7, 128.2, 121.7, 43.5, 21.79, 21.72, 20.2, 16.8. HRMS (ESI) Calcd. for $\mathrm{C}_{22} \mathrm{H}_{23} \mathrm{O}_{2}\left(\mathrm{M}+\mathrm{H}^{+}\right) \mathrm{m} / \mathrm{z} 319.1692$, found 319.1692 .<smiles>COc1ccc(C(=O)C=CC2(C=CC(=O)c3ccc(OC)cc3)CC2)cc1</smiles>

\section{(E)-1-(4-methoxyphenyl)-3-(1-(2-(4-methoxyphenyl)-2-}

oxoethyl)cyclopropyl)prop-2-en-1-one (5c): Crude product was purified by flash column chromatography $\left(\mathrm{SiO}_{2}\right.$, petroleum ether/ether $\left.=10: 1 \rightarrow 1: 1\right)$. Yield 68\% $(138$ mg); white solid; m. p. $=128-131^{\circ} \mathrm{C}$. IR (ATR): 3074, 3012, 2965, 2938, 1678, 1659, 1600, 1575, 1510, 1461, 1420, 1355, 1311, 1251, 1216, 1170, 1025, $982 \mathrm{~cm}^{-1} .{ }^{1} \mathrm{H}$ NMR $\left(500 \mathrm{MHz}, \mathrm{CDCl}_{3}\right) \delta 7.97(\mathrm{~d}, J=8.9 \mathrm{~Hz}, 2 \mathrm{H}), 7.81-7.78(\mathrm{~m}, 2 \mathrm{H}), 6.96(\mathrm{~d}, J=8.9 \mathrm{~Hz}$, 2H), $6.86(\mathrm{~d}, J=8.9 \mathrm{~Hz}, 2 \mathrm{H}), 6.71(\mathrm{~d}, J=15.4 \mathrm{~Hz}, 1 \mathrm{H}), 6.65(\mathrm{~d}, J=15.4 \mathrm{~Hz}, 1 \mathrm{H}), 3.88$ (s, 3H), 3.83 (s, 3H), 3.24 (s, 2H), $1.12-1.10(\mathrm{~m}, 2 \mathrm{H}), 1.03-1.01(\mathrm{~m}, 2 \mathrm{H}) .{ }^{13} \mathrm{C}$ NMR $\left(126 \mathrm{MHz}, \mathrm{CDCl}_{3}\right) \delta 196.1,188.6,163.7,163.2,155.2,131.2,130.8,130.46,130.42$, 121.4, 114.0, 113.7, 55.6, 55.5, 43.2, 20.2, 16.7. HRMS (ESI) Calcd. for $\mathrm{C}_{22} \mathrm{H}_{23} \mathrm{O}_{4}$ $\left(\mathrm{M}+\mathrm{H}^{+}\right) \mathrm{m} / \mathrm{z} 351.1590$, found 351.1592 .<smiles>O=C(/C=C/C1(CC(=O)c2ccc(Cl)cc2)CC1)c1ccc(Cl)cc1</smiles>

(E)-1-(4-chlorophenyl)-3-(1-(2-(4-chlorophenyl)-2-oxoethyl)cyclopropyl)prop-2-

en-1-one (5h): Crude product was purified by flash column chromatography $\left(\mathrm{SiO}_{2}\right.$, petroleum ether/ether $=10: 1 \rightarrow 1: 1)$. Yield 68\% (141 mg); yellow semi-solid. IR (ATR): 3072, 3004, 2908, 1684, 1659, 1600, 1589, 1488, 1398, 1365, 1352, 1257, 
1213, 1178, 1091, 1012, 985, 938, 824, $767 \mathrm{~cm}^{-1} .{ }^{1} \mathrm{H}$ NMR (500 MHz, $\left.\mathrm{CDCl}_{3}\right) \delta 7.91$ $(\mathrm{d}, J=8.6 \mathrm{~Hz}, 2 \mathrm{H}), 7.73(\mathrm{~d}, J=8.5 \mathrm{~Hz}, 2 \mathrm{H}), 7.47(\mathrm{~d}, J=8.6 \mathrm{~Hz}, 2 \mathrm{H}), 7.37(\mathrm{~d}, J=8.5$ $\mathrm{Hz}, 2 \mathrm{H}), 6.74(\mathrm{~d}, J=15.5 \mathrm{~Hz}, 1 \mathrm{H}), 6.56(\mathrm{~d}, J=15.5 \mathrm{~Hz}, 1 \mathrm{H}), 3.25$ (s, 2H), $1.14-1.13$ (m, 2H), $1.05-1.03(\mathrm{~m}, 2 \mathrm{H}) .{ }^{13} \mathrm{C}$ NMR $\left(126 \mathrm{MHz}, \mathrm{CDCl}_{3}\right) \delta 196.2,188.9,156.5$, 140.0, 138.9, 136.6, 135.4, 129.9, 129.5, 129.2, 128.8, 121.3, 43.7, 20.1, 17.1. HRMS (ESI) Calcd. for $\mathrm{C}_{20} \mathrm{H}_{17} \mathrm{Cl}_{2} \mathrm{O}_{2}\left(\mathrm{M}+\mathrm{H}^{+}\right) \mathrm{m} / \mathrm{z}$ 359.0606, found 359.0604 .

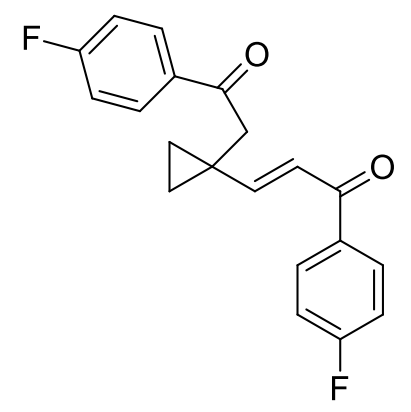

(E)-1-(4-fluorophenyl)-3-(1-(2-(4-fluorophenyl)-2-oxoethyl)cyclopropyl)prop-2en-1-one (5i): Crude product was purified by flash column chromatography $\left(\mathrm{SiO}_{2}\right.$, petroleum ether/ether $=10: 1 \rightarrow 1: 1)$. Yield 74\% $(140 \mathrm{mg})$; white solid; $\mathrm{m} . \mathrm{p} .=145-147$ ${ }^{\circ} \mathrm{C}$. IR (ATR): 3077, 3009, 1687, 1662, 1602, 1510, 1420, 1409, 1365, 1355, $1259,1227,1216,1156,1017,987,936,832,751 \mathrm{~cm}^{-1} .{ }^{1} \mathrm{H} \mathrm{NMR}\left(500 \mathrm{MHz}, \mathrm{CDCl}_{3}\right) \delta$ $8.03-7.99(\mathrm{~m}, 2 \mathrm{H}), 7.83-7.81(\mathrm{~m}, 2 \mathrm{H}), 7.18-7.14(\mathrm{~m}, 2 \mathrm{H}), 7.08-7.04(\mathrm{~m}, 2 \mathrm{H})$, $6.74(\mathrm{~d}, J=15.4 \mathrm{~Hz}, 1 \mathrm{H}), 6.58(\mathrm{~d}, J=15.5 \mathrm{~Hz}, 1 \mathrm{H}), 3.26(\mathrm{~s}, 2 \mathrm{H}), 1.16-1.13(\mathrm{~m}, 2 \mathrm{H})$, $1.04-1.02(\mathrm{~m}, 2 \mathrm{H}) .{ }^{13} \mathrm{C}$ NMR $\left(126 \mathrm{MHz}, \mathrm{CDCl}_{3}\right) \delta 195.8,188.6,166.7(\mathrm{~d}, J=65.7$ $\mathrm{Hz}), 164.7$ (d, $J=64.2 \mathrm{~Hz}), 156.2,134.6(\mathrm{~d}, J=2.9 \mathrm{~Hz}), 133.5$ (d, $J=3.1 \mathrm{~Hz}), 131.0$ $(\mathrm{d}, J=9.2 \mathrm{~Hz}), 130.7(\mathrm{~d}, J=9.3 \mathrm{~Hz}), 121.3,116.0(\mathrm{~d}, J=21.9 \mathrm{~Hz}), 115.6(\mathrm{~d}, J=21.8$ $\mathrm{Hz}$ ), 43.7, 20.0, 17.0. HRMS (ESI) Calcd. for $\mathrm{C}_{20} \mathrm{H}_{17} \mathrm{~F}_{2} \mathrm{O}_{2}\left(\mathrm{M}+\mathrm{H}^{+}\right) \mathrm{m} / \mathrm{z} 327.1191$, found 327.1194 .<smiles>O=C(/C=C/C1(CC(=O)c2ccc([N+](=O)[O-])cc2)CC1)c1ccc([N+](=O)[O-])cc1</smiles> 
(E)-1-(4-nitrophenyl)-3-(1-(2-(4-nitrophenyl)-2-oxoethyl)cyclopropyl)prop-2-en-

1-one (5j): Crude product was purified by flash column chromatography $\left(\mathrm{SiO}_{2}\right.$, petroleum ether/ether $=5: 1 \rightarrow 1: 1)$. Yield 75\% $(165 \mathrm{mg})$; orange solid; $\mathrm{m} . \mathrm{p} .=166-170$ ${ }^{\circ}$ C. IR (ATR): 3310, 3077, 3047, 3017, 2911, 1692, 1692, 1662, 1602, 1589, 1518, 1417, 1344, 1319, 1254, 1208, 1107, 1072, $990 \mathrm{~cm}^{-1} .{ }^{1} \mathrm{H}$ NMR $\left(500 \mathrm{MHz}, \mathrm{CDCl}_{3}\right) \delta$ $8.34(\mathrm{~d}, J=8.4 \mathrm{~Hz}, 2 \mathrm{H}), 8.26(\mathrm{~d}, J=8.4 \mathrm{~Hz}, 2 \mathrm{H}), 8.12(\mathrm{~d}, J=8.0 \mathrm{~Hz}, 2 \mathrm{H}), 7.95(\mathrm{~d}, J$ $=8.0 \mathrm{~Hz}, 2 \mathrm{H}), 6.82(\mathrm{~d}, J=15.5 \mathrm{~Hz}, 1 \mathrm{H}), 6.56(\mathrm{~d}, J=15.5 \mathrm{~Hz}, 1 \mathrm{H}), 3.35(\mathrm{~s}, 2 \mathrm{H}), 1.25$ - 1.19 (m, 2H), 1.12 - 1.08 (m, 2H). ${ }^{13} \mathrm{C}$ NMR (126 MHz, $\left.\mathrm{CDCl}_{3}\right) \delta$ 195.7, 188.8, 157.9, 150.7, 150.0, 143.2, 141.1, 129.4, 129.1, 124.2, 123.8, 121.4, 44.6, 19.9, 17.6. HRMS (ESI) Calcd. for $\mathrm{C}_{20} \mathrm{H}_{17} \mathrm{~N}_{2} \mathrm{O}_{6}\left(\mathrm{M}+\mathrm{H}^{+}\right) \mathrm{m} / \mathrm{z} 381.1081$, found 381.1082 .

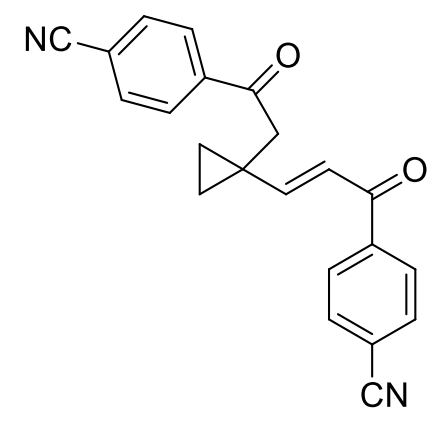

(E)-4-(3-(1-(2-(4-cyanophenyl)-2-oxoethyl)cyclopropyl)acryloyl)benzonitrile (5k):

Crude product was purified by flash column chromatography $\left(\mathrm{SiO}_{2}\right.$, petroleum ether/ether $=5: 1 \rightarrow 1: 1)$. Yield 65\% (128 mg); yellow semi-solid. IR (ATR): 3080, 3055, 3004, 2919, 2231, 1692, 1660, 1594, 1450, 1423, 1404, 1371, 1357, 1292, 1259, 1213, 1180, $990 \mathrm{~cm}^{-1} .{ }^{1} \mathrm{H}$ NMR (500 MHz, $\left.\mathrm{CDCl}_{3}\right) \delta 8.05(\mathrm{~d}, J=8.2 \mathrm{~Hz}, 2 \mathrm{H}), 7.88(\mathrm{~d}$, $J=8.1 \mathrm{~Hz}, 2 \mathrm{H}), 7.80(\mathrm{~d}, J=8.2 \mathrm{~Hz}, 2 \mathrm{H}), 7.71(\mathrm{~d}, J=8.2 \mathrm{~Hz}, 2 \mathrm{H}), 6.79(\mathrm{~d}, J=15.5$ $\mathrm{Hz}, 1 \mathrm{H}), 6.53$ (d, J = 15.5 Hz, 1H), 3.30 (s, 2H), $1.21-1.19$ (m, 2H), 1.07 - 1.05 (m, 2H). ${ }^{13} \mathrm{C}$ NMR $\left(126 \mathrm{MHz}, \mathrm{CDCl}_{3}\right) \delta 195.9,188.9,157.6,141.6,139.7,132.8,132.4$, 128.9, 128.5, 121.2, 118.1, 117.8, 116.9, 115.8, 44.3, 19.9, 17.5. HRMS (ESI) Calcd. for $\mathrm{C}_{22} \mathrm{H}_{17} \mathrm{~N}_{2} \mathrm{O}_{2}\left(\mathrm{M}+\mathrm{H}^{+}\right) \mathrm{m} / \mathrm{z} 341.1284$, found 341.1284.

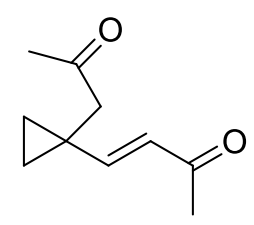


(E)-4-(1-(2-oxopropyl)cyclopropyl)but-3-en-2-one (5s): Crude product was purified by flash column chromatography $\left(\mathrm{SiO}_{2}\right.$, petroleum ether/ether $\left.=10: 1 \rightarrow 5: 1\right)$. Yield 54\% (52 mg); yellow oil. IR (ATR): 3082, 3007, 2903, 1712, 1663, 1608, 1424, 1361, 1260, 1168, 981, $915 \mathrm{~cm}^{-1} .{ }^{1} \mathrm{H}$ NMR $\left(500 \mathrm{MHz}, \mathrm{CDCl}_{3}\right) \delta 6.35(\mathrm{~d}, J=16.1 \mathrm{~Hz}, 1 \mathrm{H}), 5.82(\mathrm{~d}$, $J=16.0 \mathrm{~Hz}, 1 \mathrm{H}), 2.55(\mathrm{~s}, 2 \mathrm{H}), 2.13(\mathrm{~s}, 3 \mathrm{H}), 2.11(\mathrm{~s}, 3 \mathrm{H}), 0.97(\mathrm{t}, J=5.8 \mathrm{~Hz}, 2 \mathrm{H}), 0.91$ $(\mathrm{t}, J=5.8 \mathrm{~Hz}, 2 \mathrm{H}) .{ }^{13} \mathrm{C} \mathrm{NMR}\left(126 \mathrm{MHz}, \mathrm{CDCl}_{3}\right) \delta 206.4,197.5,153.8,126.7,49.1$, 29.8, 27.2, 19.2, 16.8. HRMS (ESI) Calcd. for $\mathrm{C}_{10} \mathrm{H}_{15} \mathrm{O}_{2}\left(\mathrm{M}+\mathrm{H}^{+}\right) \mathrm{m} / \mathrm{z} 167.1066$, found 167.1066 .

Procedure for the preparation of 2-(1-(hydroxymethyl)cyclopropyl)-1phenylethanol (8).

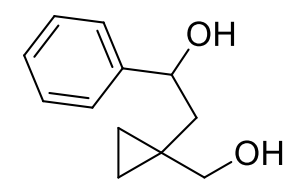

To a solution of $4 \mathbf{a}(0.25 \mathrm{mmol}, 47 \mathrm{mg})$ in $\mathrm{MeOH}(1 \mathrm{~mL})$ was added $\mathrm{NaBH}_{4}(0.5 \mathrm{mmol}$, $19 \mathrm{mg}$ ) at room temperature and the reaction mixture was stirred at the same temperature for $1 \mathrm{~h}$ under nitrogen atmosphere. The mixture was poured into $\mathrm{H}_{2} \mathrm{O}$ and extracted with EtOAc. The organic layer was washed with brine and dried with $\mathrm{Na}_{2} \mathrm{SO}_{4}$. After removal of the solvent, the residue was subjected to column chromatography $\left(\mathrm{SiO}_{2}\right.$, petroleum ether/ether $\left.=5: 1 \rightarrow 1: 1\right)$ to give 7: Yield $83 \%(40$ mg); yellow oil. IR (ATR): 3266, 3070, 3033, 3004, 2929, 2903, 1493, 1453, 1433, 1341, 1277, 1200, 1064, 1030, $912 \mathrm{~cm}^{-1} .{ }^{1} \mathrm{H}$ NMR (500 MHz, $\left.\mathrm{CDCl}_{3}\right) \delta 7.35-7.31$ (m, 4H), $7.27-7.23(\mathrm{~m}, 1 \mathrm{H}), 4.86(\mathrm{dd}, J=9.8,2.2 \mathrm{~Hz}, 1 \mathrm{H}), 3.77(\mathrm{dd}, J=11.5,0.9 \mathrm{~Hz}$, $1 \mathrm{H}), 3.41$ (br s, 1H), 3.24 (d, $J=11.5 \mathrm{~Hz}, 1 \mathrm{H}), 2.16$ (ddd, $J=15.0,9.8,1.2 \mathrm{~Hz}, 1 \mathrm{H}$ ), 1.61 (br s, 1H), $1.43-1.37(\mathrm{~m}, 1 \mathrm{H}), 0.57-0.53(\mathrm{~m}, 1 \mathrm{H}), 0.51-0.45(\mathrm{~m}, 2 \mathrm{H}), 0.42-$ $0.36(\mathrm{~m}, 1 \mathrm{H}) .{ }^{13} \mathrm{C}$ NMR $\left(126 \mathrm{MHz}, \mathrm{CDCl}_{3}\right) \delta 145.0,128.5,127.5,125.7,73.9,70.5$, 47.6, 21.8, 13.4, 9.6. HRMS (ESI) Calcd. for $\mathrm{C}_{12} \mathrm{H}_{17} \mathrm{O}_{2}\left(\mathrm{M}+\mathrm{H}^{+}\right) \mathrm{m} / \mathrm{z} 193.1223$, found 193.1227.

Procedure for the preparation of Ethyl 2-methyl-3-(1-(2-0xo-2phenylethyl)cyclopropyl)acrylate (9). 


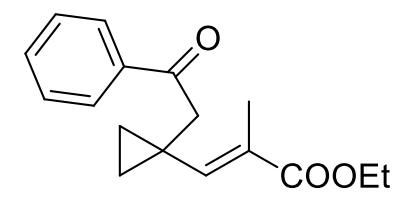

A solution of $\mathbf{4 a}(0.58 \mathrm{mmol})$ and methyl 2-(triphenylphosphoranylidene)propanoate $(0.58 \mathrm{mmol})$ in toluene $(0.5 \mathrm{~mL})$ was stirred in a sealed tube reactor at $110{ }^{\circ} \mathrm{C}$ for 48 h. The crude mixture, without aqueous work-up, was directly purified by flash column chromatography $\left(\mathrm{SiO}_{2}\right.$, petroleum ether/ether $\left.=10: 1 \rightarrow 5: 1\right)$ to give compound $\mathbf{8}$ : Yield 62\% (97 mg); yellow oil. IR (ATR): 3076, 2987, 2929, 2903, 1703, 1694, 1646, 1450, 1392, 1366, 1257, 1214, 1170, 1116, 1033, $978 \mathrm{~cm}^{-1}$. D. r. ratio 76:24. Major diastereoisomer: ${ }^{1} \mathrm{H}$ NMR $\left(500 \mathrm{MHz}, \mathrm{CDCl}_{3}\right) \delta 7.89(\mathrm{~d}, J=8.2 \mathrm{~Hz}, 2 \mathrm{H}), 7.56-7.53$ (m, 1H), $7.44(\mathrm{t}, J=7.7 \mathrm{~Hz}, 2 \mathrm{H}), 6.96(\mathrm{~s}, 1 \mathrm{H}), 4.13(\mathrm{q}, J=7.1 \mathrm{~Hz}, 2 \mathrm{H}), 3.14(\mathrm{~s}, 2 \mathrm{H})$, $1.94(\mathrm{~s}, 3 \mathrm{H}), 1.25(\mathrm{t}, J=7.1 \mathrm{~Hz}, 3 \mathrm{H}), 0.83-0.81(\mathrm{~m}, 2 \mathrm{H}), 0.79-0.77(\mathrm{~m}, 2 \mathrm{H}) .{ }^{13} \mathrm{C}$ NMR (126 MHz, $\left.\mathrm{CDCl}_{3}\right) \delta 198.1,168.5,144.0,137.1,133.2,130.6,128.6,128.2,60.6$, 47.1, 16.7, 14.3, 14.0, 13.6. HRMS (ESI) Calcd. for $\mathrm{C}_{17} \mathrm{H}_{21} \mathrm{O}_{3}\left(\mathrm{M}+\mathrm{H}^{+}\right) \mathrm{m} / \mathrm{z} 273.1485$, found 273.1481 .

The following data are attributed to the minor diastereoisomer in the mixture: ${ }^{1} \mathrm{H}$ NMR $\left(500 \mathrm{MHz}, \mathrm{CDCl}_{3}\right) \delta 4.21(\mathrm{q}, J=7.2 \mathrm{~Hz}, 2 \mathrm{H}), 1.29(\mathrm{t}, J=7.2 \mathrm{~Hz}, 3 \mathrm{H})$, other signals masked. ${ }^{13} \mathrm{C}$ NMR $\left(126 \mathrm{MHz}, \mathrm{CDCl}_{3}\right) \delta 169.0,133.4,61.1,15.7,14.1$, other signals masked.

Procedure for the preparation of methyl 1-(2-oxo-2phenylethyl)cyclopropanecarboxylate (10).

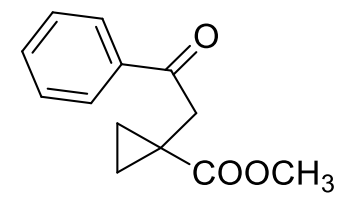

The cyclopropanecarbaldehyde $4 \mathbf{a}(0.57 \mathrm{mmol})$ was dissolved in Methanol $(6 \mathrm{~mL})$. Oxone $(0.57 \mathrm{mmol}, 174 \mathrm{mg}$ ) was added and stirred at room temperature for $48 \mathrm{~h} .1 \mathrm{~N}$ $\mathrm{HCl}$ was used to dissolve the salts and EtOAc was added to extract the products. The organic extract was washed with $1 \mathrm{~N} \mathrm{HCl}$ and brine, dried over $\mathrm{Na}_{2} \mathrm{SO}_{4}$, and the solvent was removed under reduced pressure to obtain the crude product. The crude mixture was purified by silica gel column chromatography $\left(\mathrm{SiO}_{2}\right.$, petroleum ether/ether $=$ 
$10: 1 \rightarrow 5: 1)$ to give compound 9: Yield 82\% (101 mg); yellow oil. IR (ATR): 3058, 3012, 2955, 1720, 1689, 1660, 1582, 1450, 1435, 1404, 1352, 1200, 1145, 1033, 1004, $981 \mathrm{~cm}^{-1} .{ }^{1} \mathrm{H}$ NMR $\left(500 \mathrm{MHz}, \mathrm{CDCl}_{3}\right) \delta 7.96-7.94(\mathrm{~m}, 2 \mathrm{H}), 7.57-7.54(\mathrm{~m}, 1 \mathrm{H}), 7.47$ - 7.44 (m, 2H), 3.62 (s, 3H), 3.25 (s, 2H), 1.43 (q, J=4.3 Hz, 2H), 0.82 (q, J = 4.4 Hz, 2H). ${ }^{13} \mathrm{C} \mathrm{NMR}\left(126 \mathrm{MHz}, \mathrm{CDCl}_{3}\right) \delta 197.6,175.0,137.0,133.1,128.6,128.0,52.1$, 42.9, 19.8, 15.2. HRMS (ESI) Calcd. for $\mathrm{C}_{13} \mathrm{H}_{15} \mathrm{O}_{3}\left(\mathrm{M}+\mathrm{H}^{+}\right) \mathrm{m} / \mathrm{z}$ 219.1016, found 219.1013.

Procedure for the preparation of 1-(2-oxo-2-phenylethyl)cyclopropanecarboxylic acid (11).

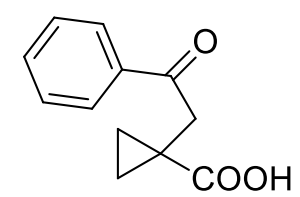

The cyclopropanecarbaldehyde $\mathbf{4 a}(0.83 \mathrm{mmol}, 156 \mathrm{mg})$ was dissolved in DMF (8.3 $\mathrm{mL})$. Oxone $(0.83 \mathrm{mmol}, 255 \mathrm{mg})$ was added in one portion and stirred at room temperature for $16 \mathrm{~h} .1 \mathrm{~N} \mathrm{HCl}$ was used to dissolve the salts and EtOAc was added to extract the products. The organic extract was washed with $1 \mathrm{~N} \mathrm{HCl}$ and brine, dried over $\mathrm{Na}_{2} \mathrm{SO}_{4}$, and the solvent was removed under reduced pressure to obtain the crude product. The crude mixture was purified by silica gel column chromatography $\left(\mathrm{SiO}_{2}\right.$, petroleum ether/ether $=1: 1)$ to give compound 10: Yield 58\% (98 mg); yellow oil. IR (ATR): 3010, 2946, 2906, 2866, 1766, 1680, 1594, 1450, 1349, 1246, 1214, 1214, 1168, 1067, $975 \mathrm{~cm}^{-1} .{ }^{1} \mathrm{H}$ NMR $\left(500 \mathrm{MHz}, \mathrm{CDCl}_{3}\right) \delta 7.95-7.93(\mathrm{~m}, 2 \mathrm{H}), 7.55(\mathrm{t}, J=$ $7.4 \mathrm{~Hz}, 1 \mathrm{H}), 7.46-7.43(\mathrm{~m}, 2 \mathrm{H}), 3.24(\mathrm{~s}, 2 \mathrm{H}), 1.49$ (q, $J=4.3 \mathrm{~Hz}, 2 \mathrm{H}), 0.88$ (q, $J=$ $4.3 \mathrm{~Hz}, 2 \mathrm{H}) .{ }^{13} \mathrm{C} \mathrm{NMR}\left(126 \mathrm{MHz}, \mathrm{CDCl}_{3}\right) \delta 197.3,180.4,136.9,133.2,128.7,128.1$, 42.6, 19.6, 15.9. HRMS (ESI) Calcd. for $\mathrm{C}_{12} \mathrm{H}_{13} \mathrm{O}_{3}\left(\mathrm{M}+\mathrm{H}^{+}\right) \mathrm{m} / \mathrm{z}$ 205.0859, found 205.0857 .

Procedure for the preparation of 1-(1-bromo-2-oxo-2phenylethyl)cyclopropanecarbaldehyde (12).

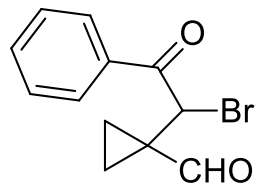


Aldehyde 4a $(0.37 \mathrm{mmol}, 70 \mathrm{mg})$ and DMSO $(31 \mu \mathrm{L})$ were dissolved in EtOAc $(4 \mathrm{~mL})$. Aqueous hydrobromic acid $47 \%\left(35 \mathrm{mg}\right.$ ) was added to the solution at $60^{\circ} \mathrm{C}$ and the mixture were stirred for $1 \mathrm{~h}$ under air at that temperature. After cooling down to room temperature and concentrating in vacuum, the residue was purified by chromatography on silica gel $\left(\mathrm{SiO}_{2}\right.$, petroleum ether/ethyl acetate $\left.=1: 1\right)$ to afford the brominated product 11: Yield 77\% (75 mg); orange oil. IR (ATR): 3067, 3007, 2869, 1683, 1597, 1579, 1450, 1364, 1303, 1266, 1208, 1035, $978 \mathrm{~cm}^{-1} .{ }^{1} \mathrm{H}$ NMR $\left(500 \mathrm{MHz}, \mathrm{CDCl}_{3}\right) \delta$ $9.40(\mathrm{~s}, 1 \mathrm{H}), 8.01-7.99(\mathrm{~m}, 2 \mathrm{H}), 7.63-7.60(\mathrm{~m}, 1 \mathrm{H}), 7.51-7.48(\mathrm{~m}, 2 \mathrm{H}), 5.41(\mathrm{~s}$, 1H), 1.59 (ddd, $J=9.4,7.5,4.8 \mathrm{~Hz}, 1 \mathrm{H}), 1.44-1.38$ (m, 2H), 1.28 (ddd, $J=9.1,7.5$, $4.8 \mathrm{~Hz}, 1 \mathrm{H}) .{ }^{13} \mathrm{C} \mathrm{NMR}\left(126 \mathrm{MHz}, \mathrm{CDCl}_{3}\right) \delta 199.1,191.9,134.16,134.13,129.0,129.0$, 47.4, 33.2, 16.3, 15.3. HRMS (ESI) Calcd. for $\mathrm{C}_{12} \mathrm{H}_{12} \mathrm{BrO}_{2}\left(\mathrm{M}+\mathrm{H}^{+}\right) \mathrm{m} / \mathrm{z}$ 267.0015, found 267.0015 . 


\section{References:}

(1) (a) Hazelard. D.; Fadel, A.; Morgant, G., Tetrahedron: Asymmetry, 2004, 15, 1711-1718; (b) Conia, J. M.; Barnier, J. P., Terahedron Lett., 1971, 4981-4984; (c) Barnier, J. P.; Denis, J. M.; Salaun, J.; Conia, J. M. Tetrahedron, 1974, 30, 1405-1411; (d) Bloomfield, J. J.; Nelke, J. M., Org. Synth. 1977, 57, 1.

(2) Porcu, S.; Luridiana, A.; Martis, A.; Frongia, A.; Sarais, G.; Aitken, D. J.; Boddaert, T.; Guillot, R.; Secci, F., Chem. Commun., 2018, 54, 13547-13550.

(3) (a) Teske, J; Plietker, B, Org. Lett. 2018, 8, 2257-2260; (b) Fukata, Y; Asano, K.; Matsubara, S., J. Am. Chem. Soc. 2013, 135, 12160-12163; (c) Yang, J.; Felton, G. A. N.; Bauld, N. L.; Krische, M. J., J. am. Chem. Soc. 2004, 126, 1634-1635; (d) Cullen, A.; Mullera, A. J.; Bradley, D.; Williams, G., RSC Adv., 2017, 7, 42168-42171; (e) Stark, D. G.; Morrill, L. C.; Yeh, P.-P.; Slawin, A. M. Z.; O'Riordan, T. J. C.; Smith, A. D., Angew. Chem. Int. Ed. 2013, 52, 11642-11646; (f) Matviitsuk, A.; Taylor, J. E.; Cordes, D. B.; Slawin, A. M. Z.; Smith, A. D., Chem. Eur. J. 2016, 22, 17748-17757; (g) An, X.-L.; Chen, J.-R.; Li, C.-F.; Zhang, F.-G.; Zou, Y.-Q.; Guo, Y.-C.; Xiao, W.-J., Chem. Asian J., 2010, 10, 2258-2265; (h) Wu, J.-Q.; Yang, Z.; Zhang, S.-S.; Jiang, C.-Y.; Li, Q.; Huang, Z.-S.; Wang, H., ACS Catalysis, 2015, 11, 6453-6457; (i) Nath, U.; Chowdhury, D.; Pan, S. C., Adv. Synth. Catal., 2018, 8, 1628-1633; (j) Dischmann, M.; Frassetto, T.; Breuning, M.; Koert, U., Chem. Eur. J., 2014, 36, 11300-11302; (k) Shinde, D. B.; Kanth, B. S.; Satyakumar, A.; Kamble, V. T.; Das, B., Lett. Org. Chem, 2013, 5, 317-323; (1) Ruijter, E.; Schültingkemper, H.; Ludger A. Wessjohann, L. A., J. Org. Chem. 2005, 707, 2820-2823; (m) Lin, L.; Romano, C.; Mazet, C., J. Am. Chem. Soc., 2016, 138, 10344-10350; (o) Wei, H.; Li, Y.; Xiao, K.; Cheng, B.; Wang, H.; Hu, L.; Zhai, H., Org. Lett., 2015, 24, 5974-5977. 


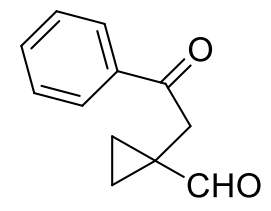

$4 a$
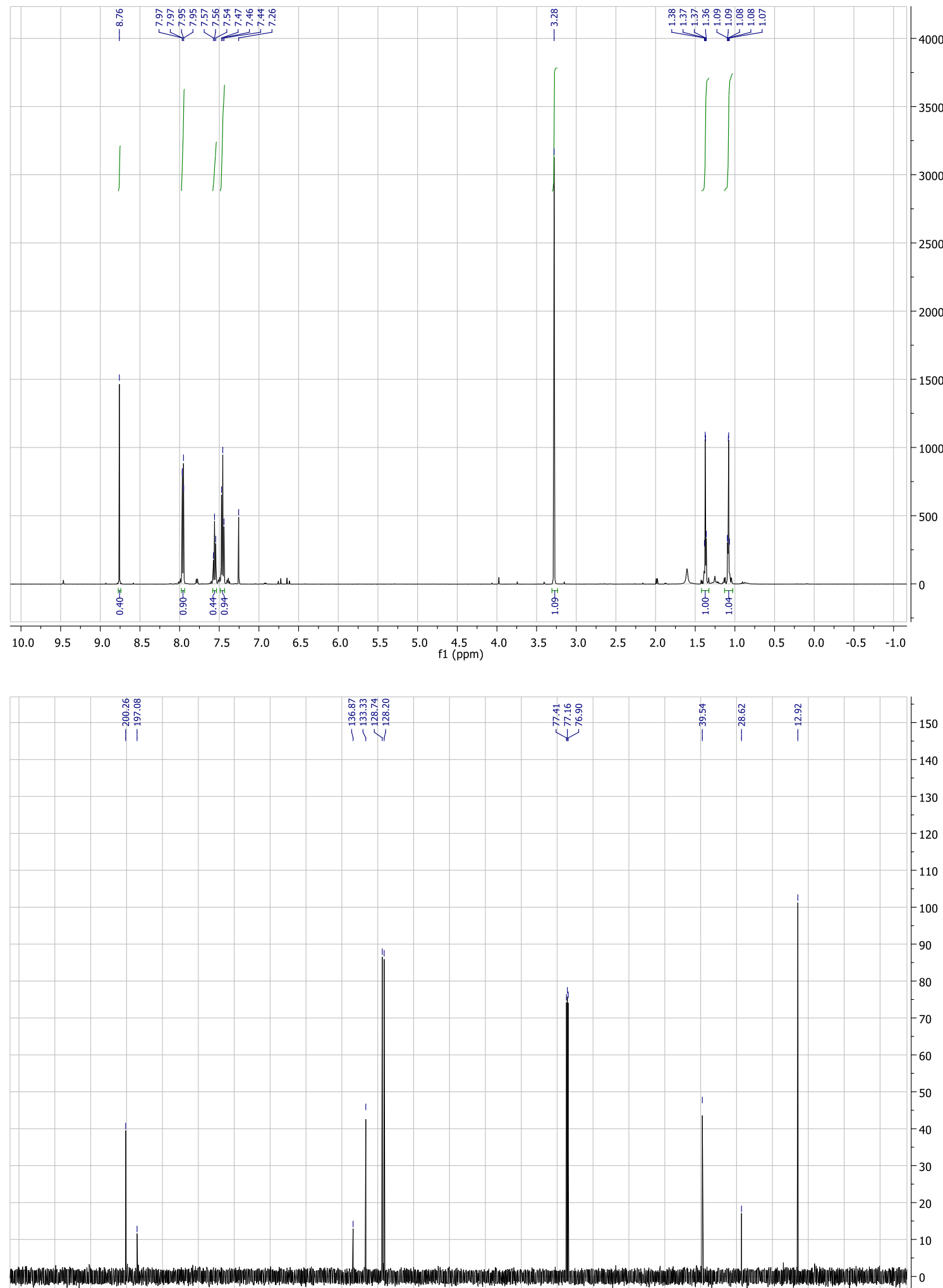

\begin{tabular}{llllllllllllllllllllllllllll}
\hline 230 & 220 & 210 & 200 & 190 & 180 & 170 & 160 & 150 & 140 & 130 & 120 & 110 & 100 & 90 & 80 & 70 & 60 & 50 & 40 & 30 & 20 & 10 & 0 & -10
\end{tabular} 


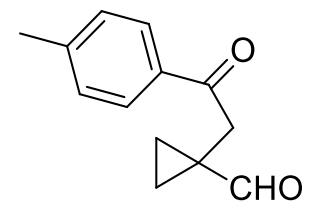

4b
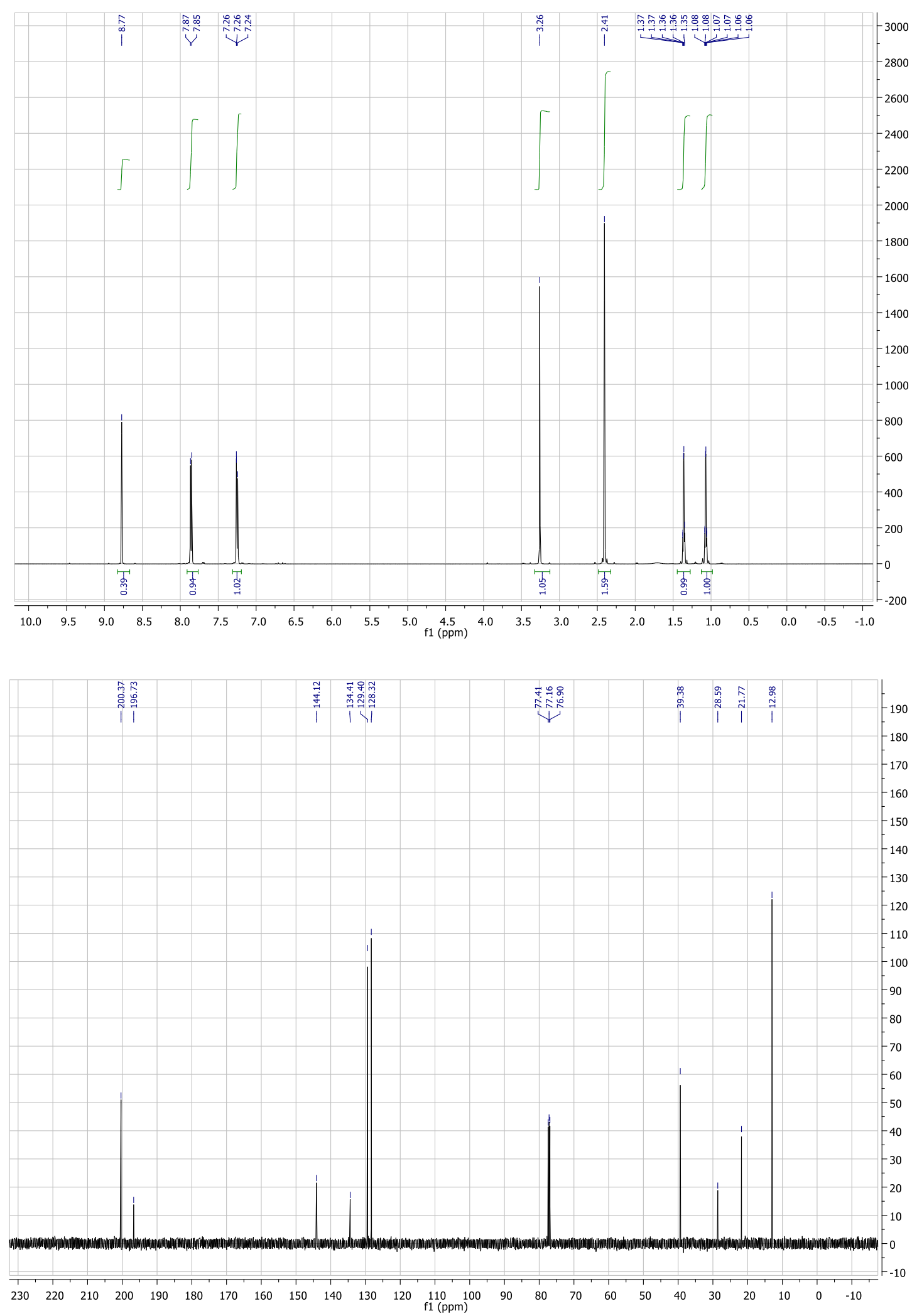

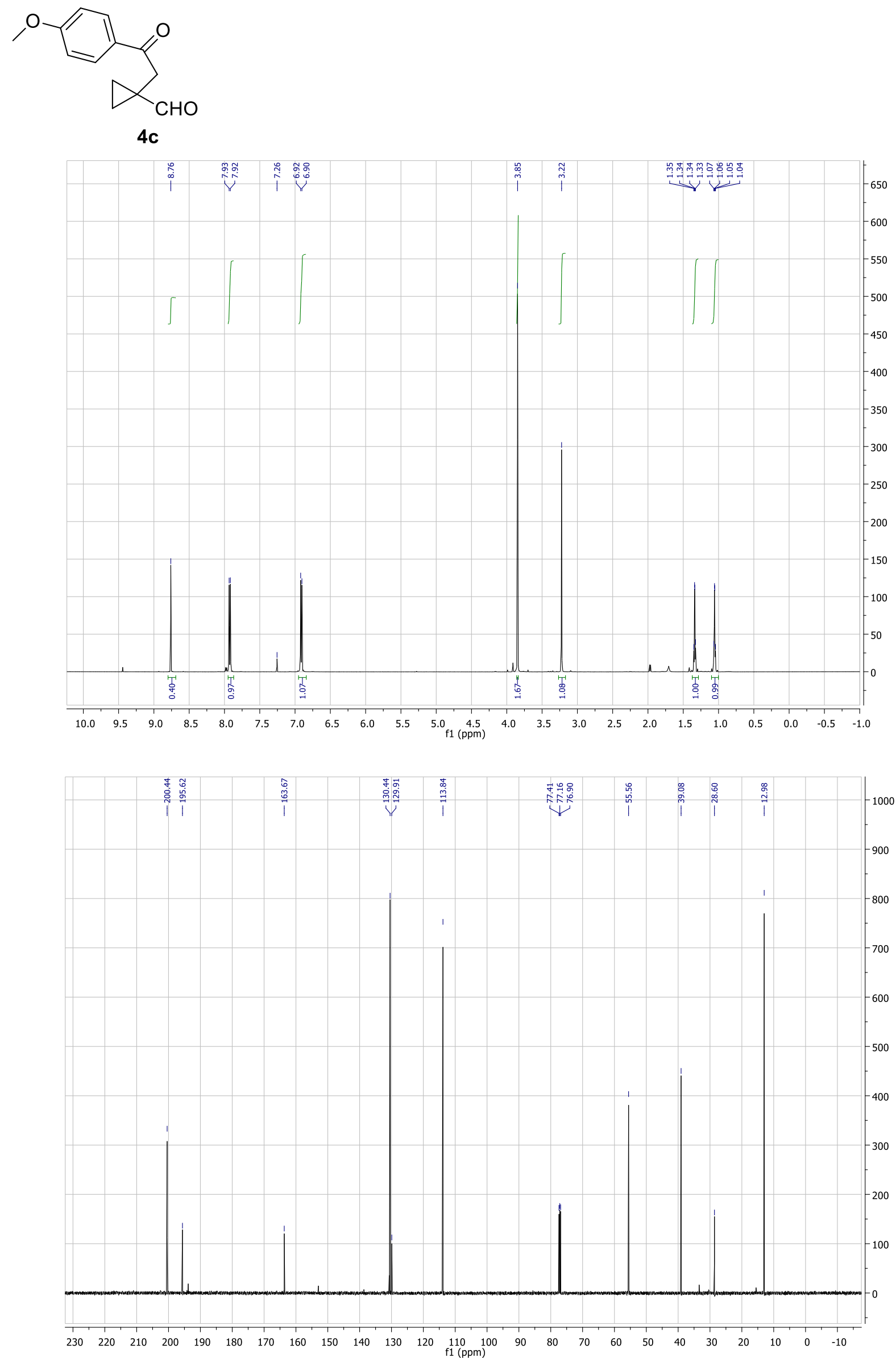
<smiles>O=CC1(CC(=O)c2ccc(OCc3ccccc3)cc2)CC1</smiles>

4d
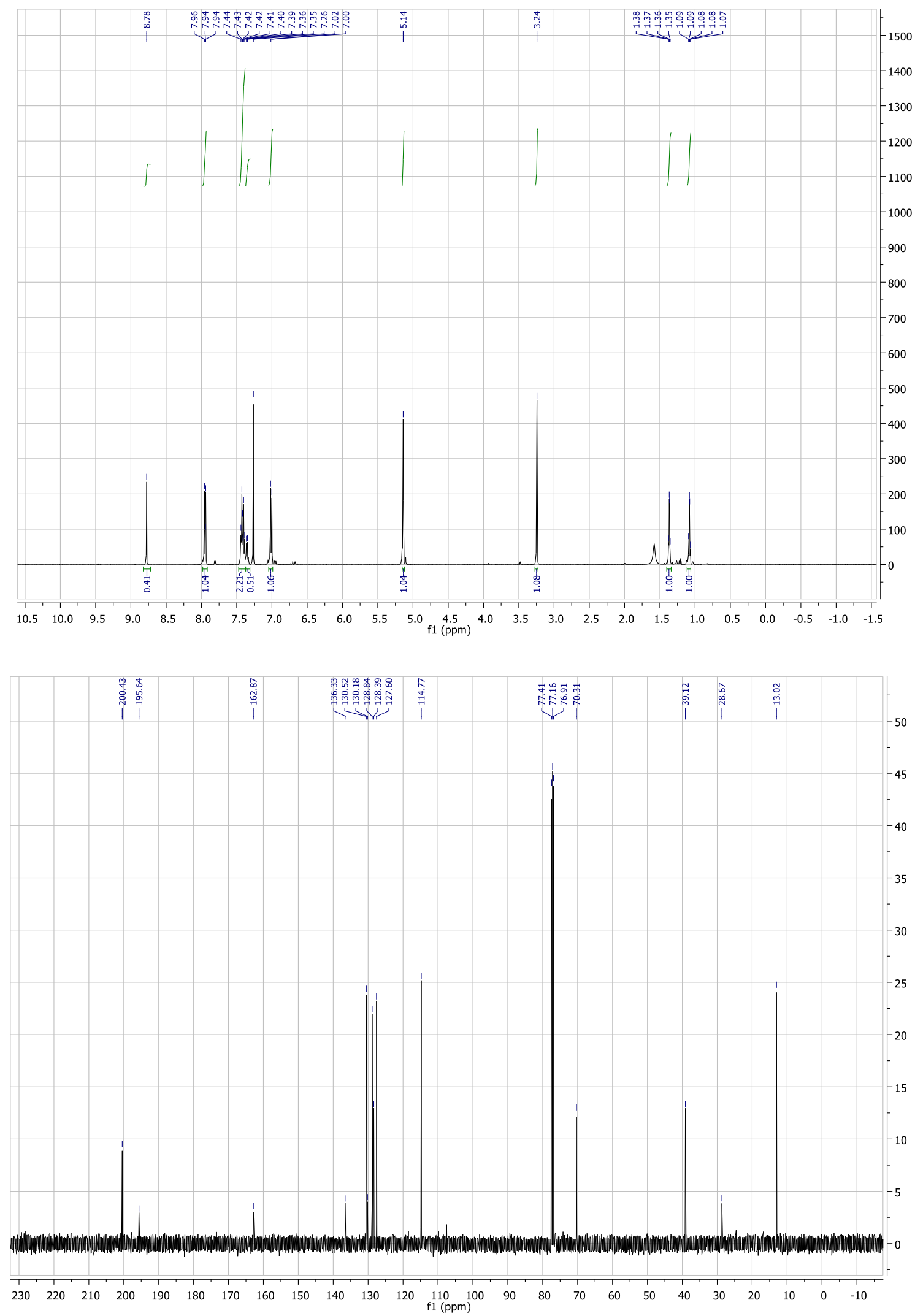

31 


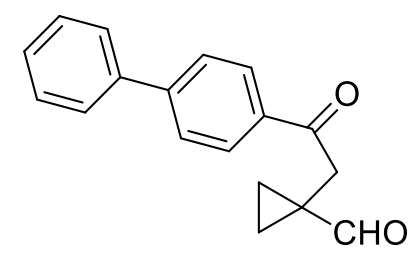

$4 e$
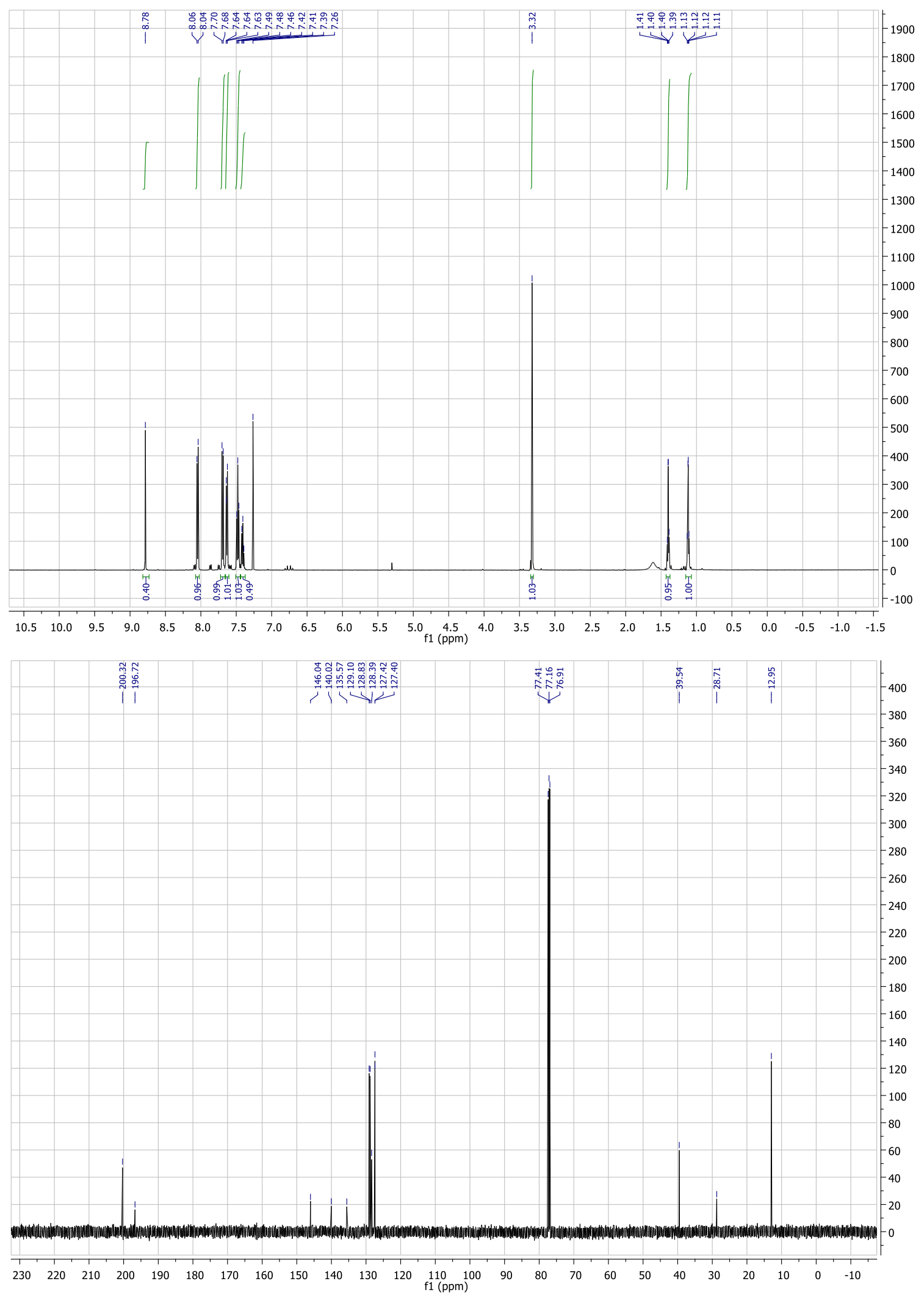

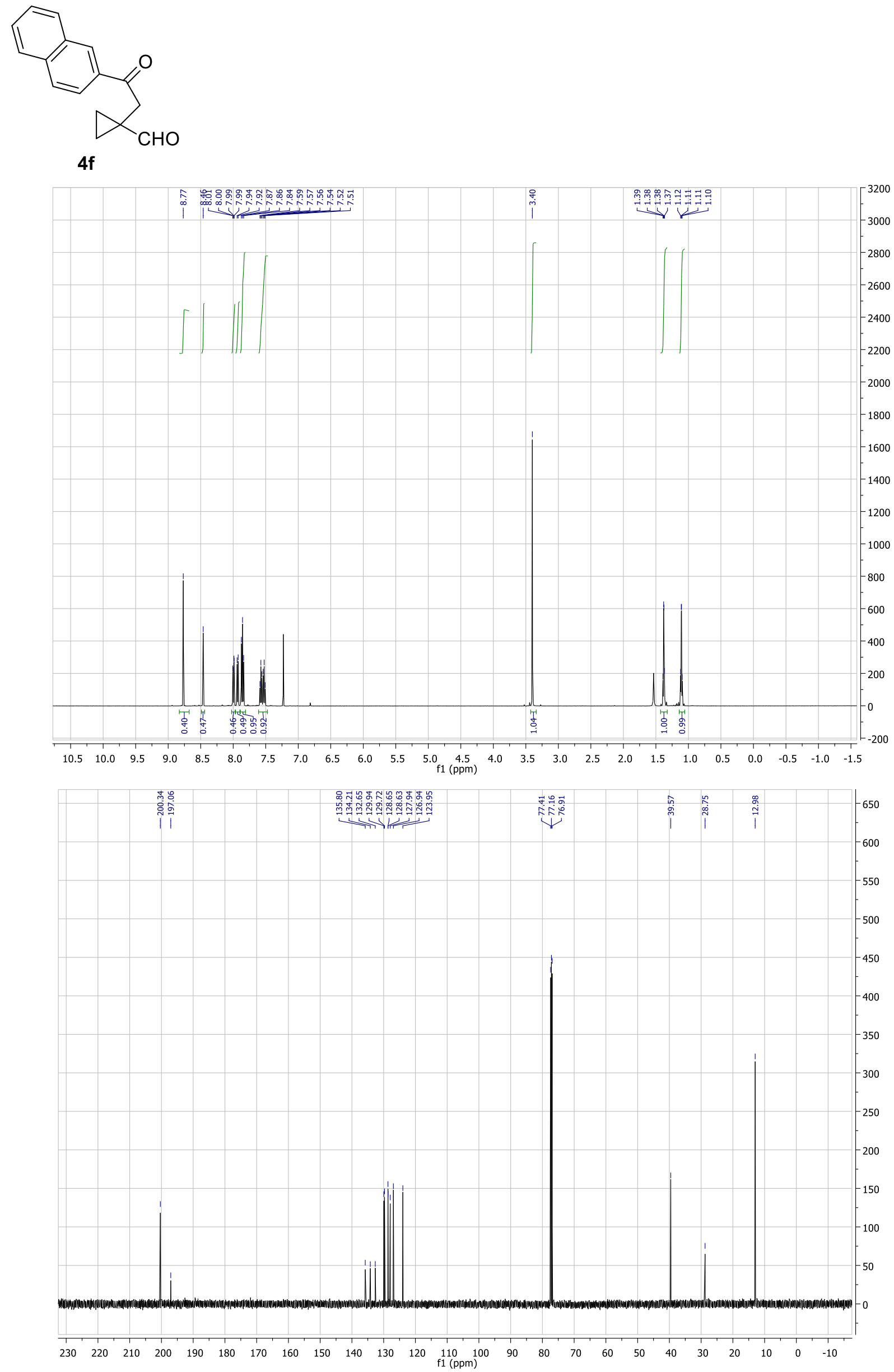
<smiles>O=CC1(CC(=O)c2ccc(Br)cc2)CC1</smiles>

$4 \mathrm{~g}$
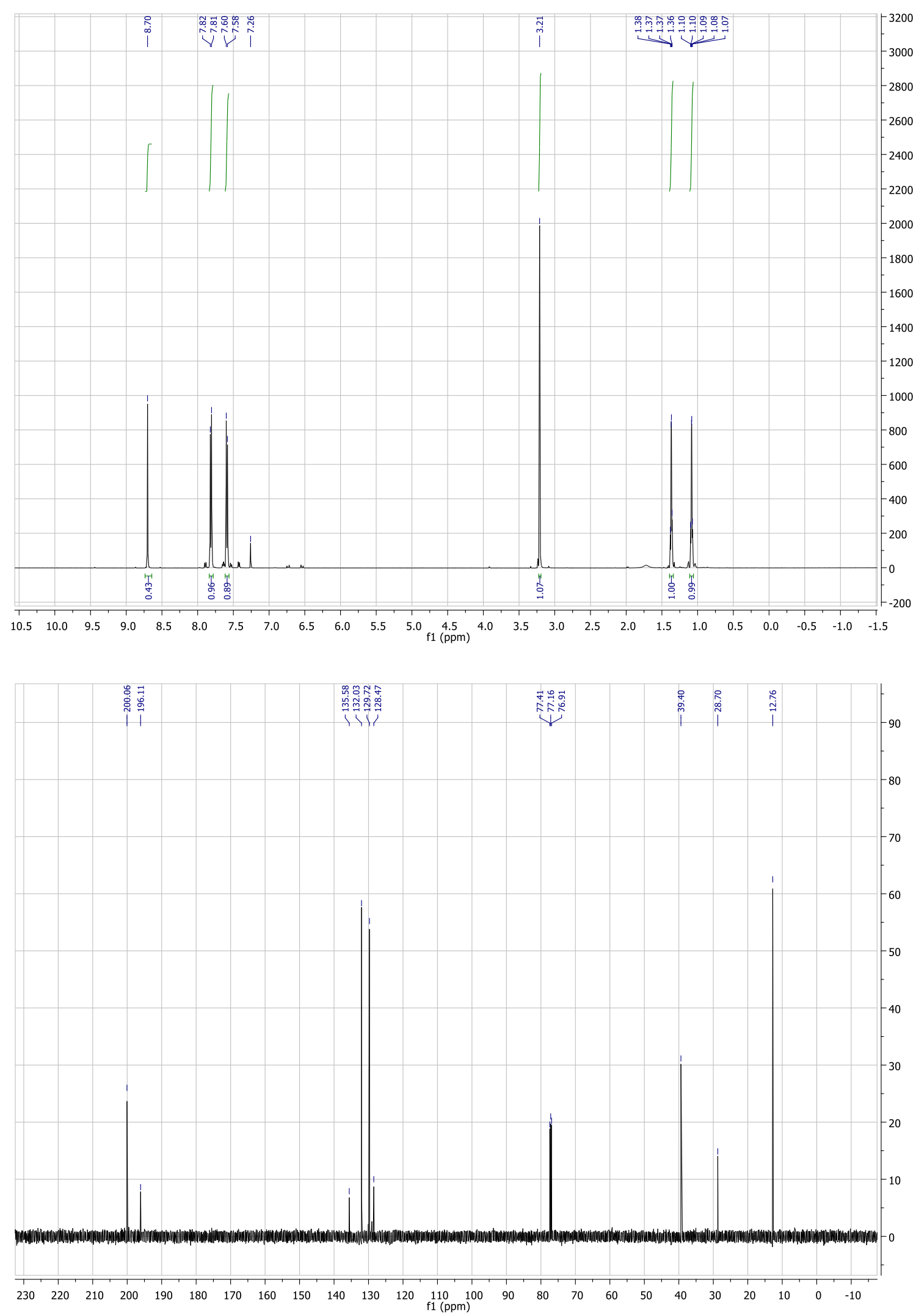


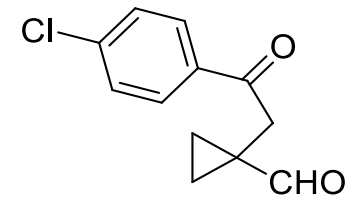

$4 h$
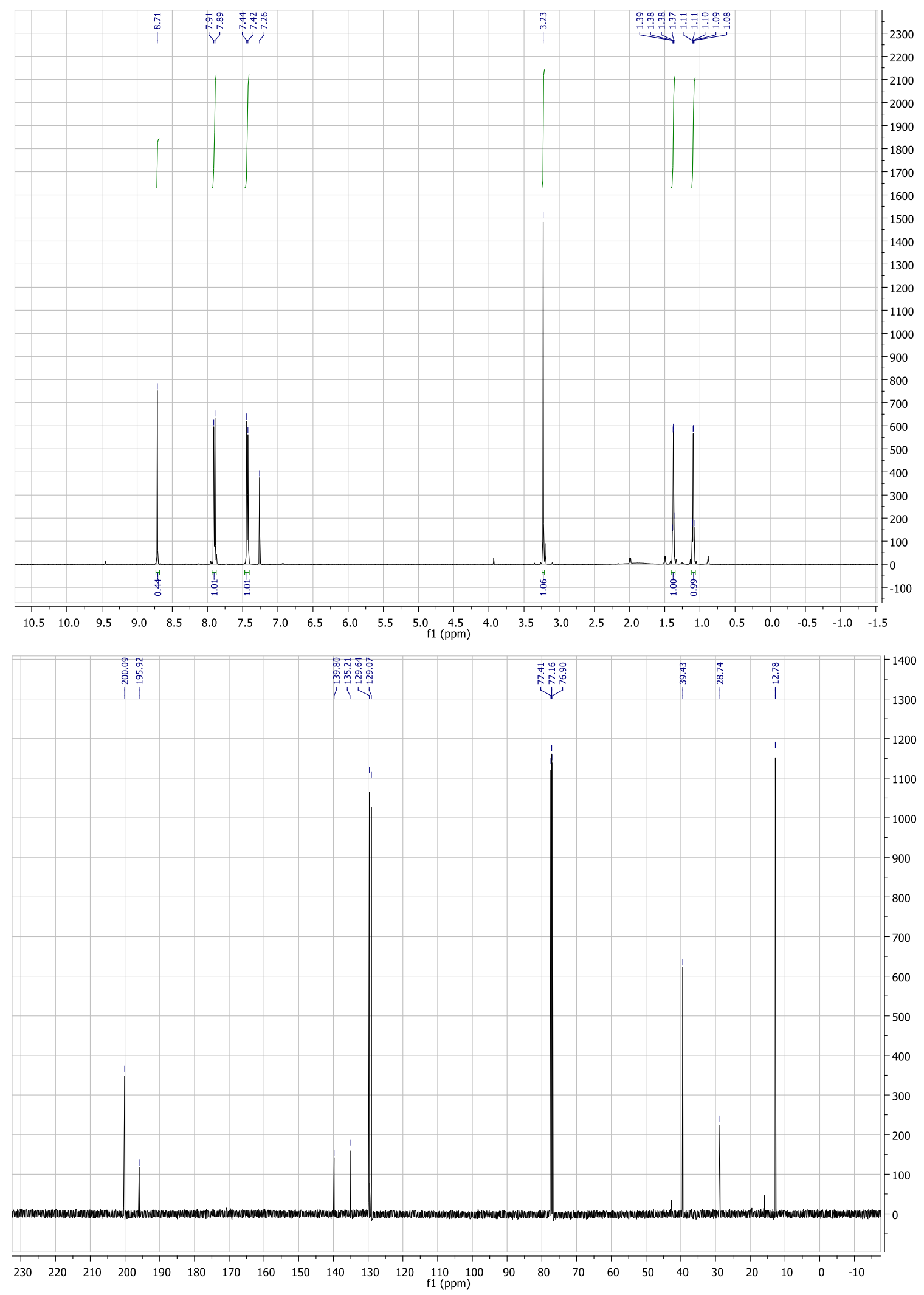


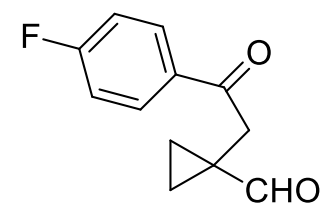

$4 i$
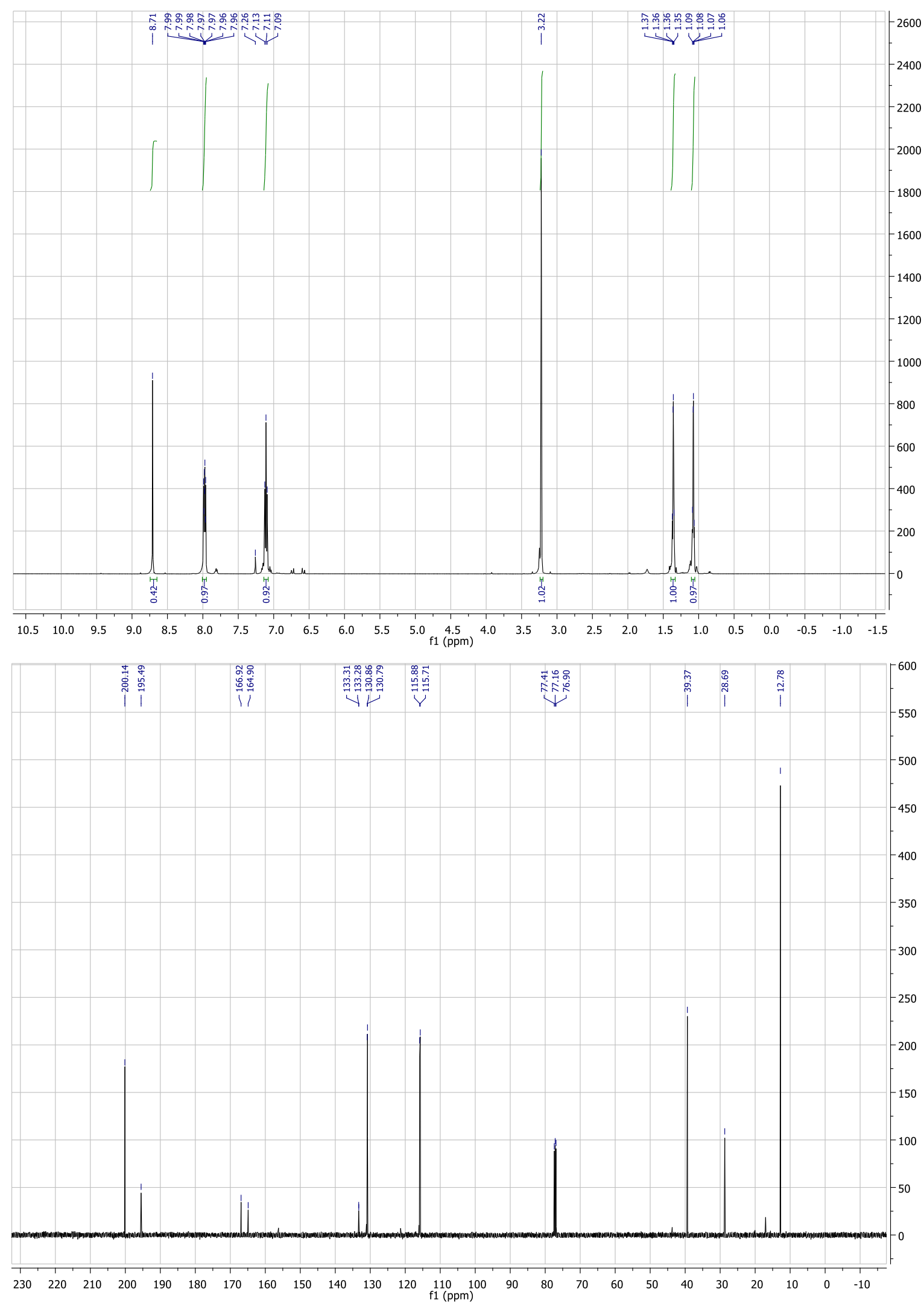


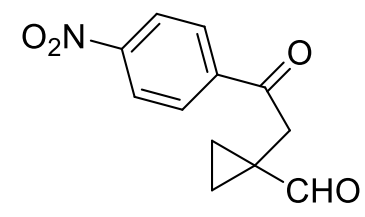

4j
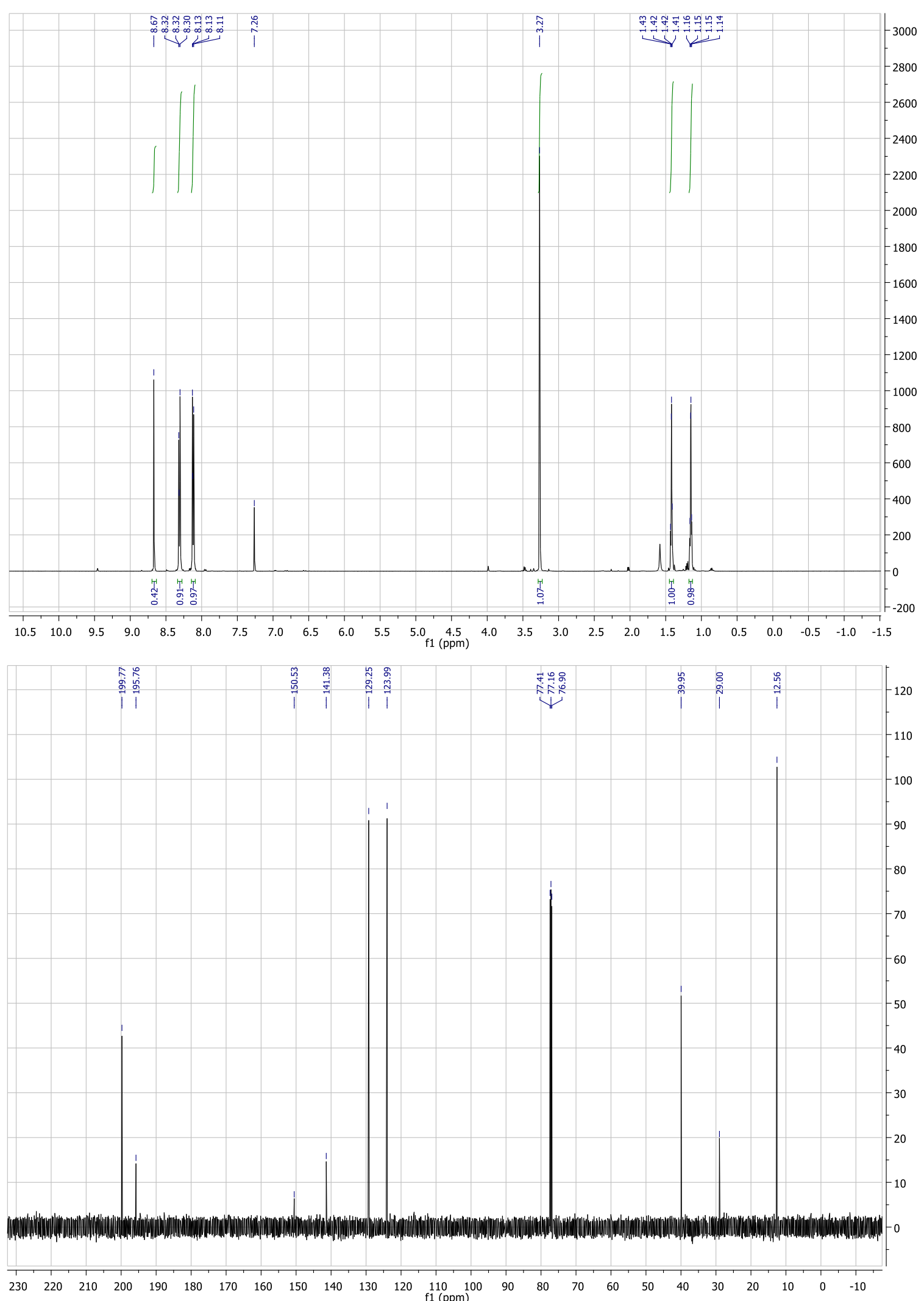


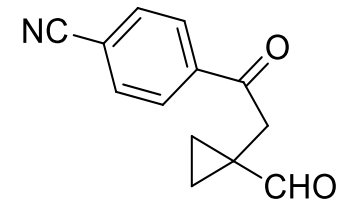

4k
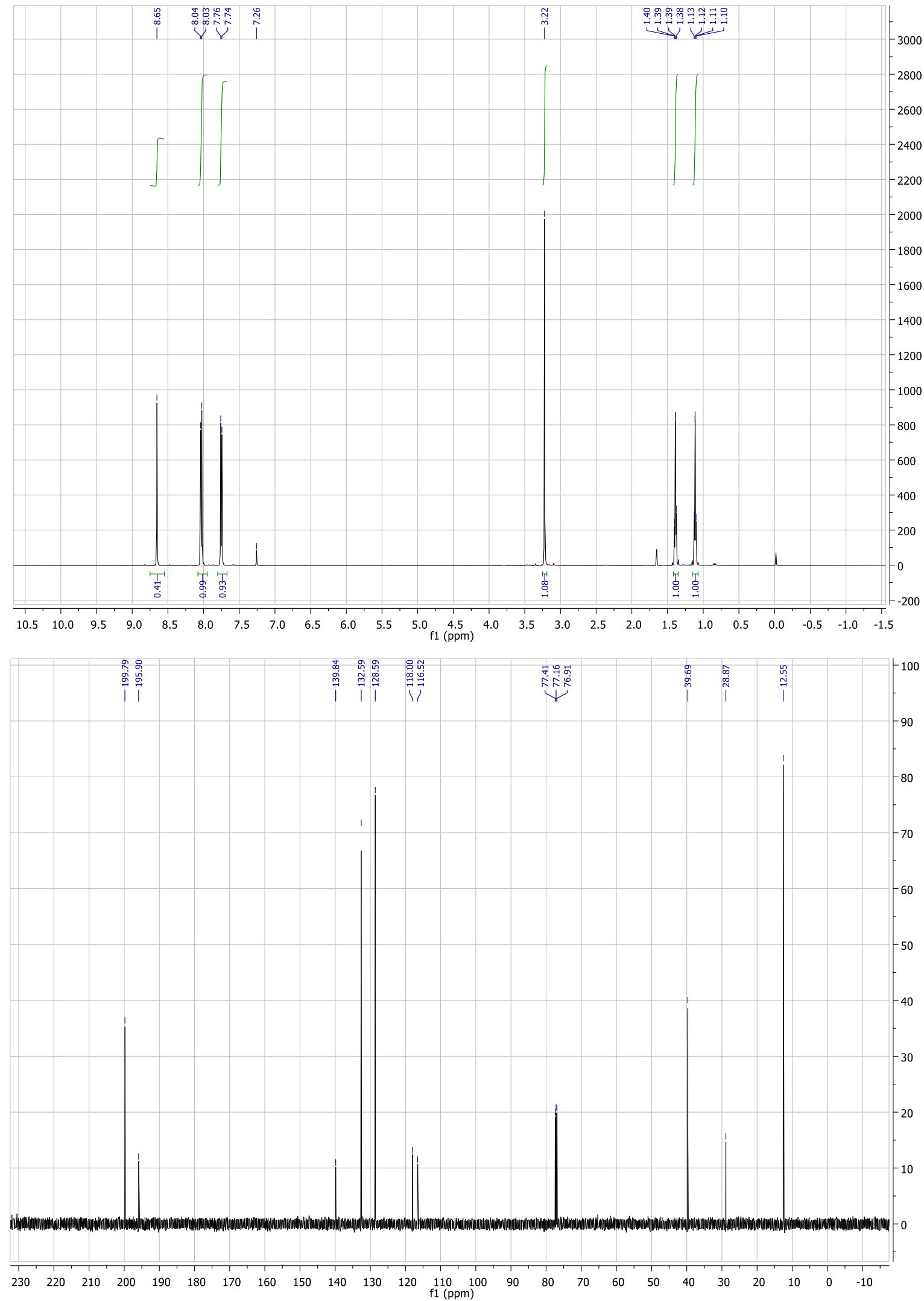


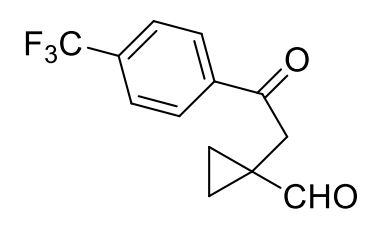

4I
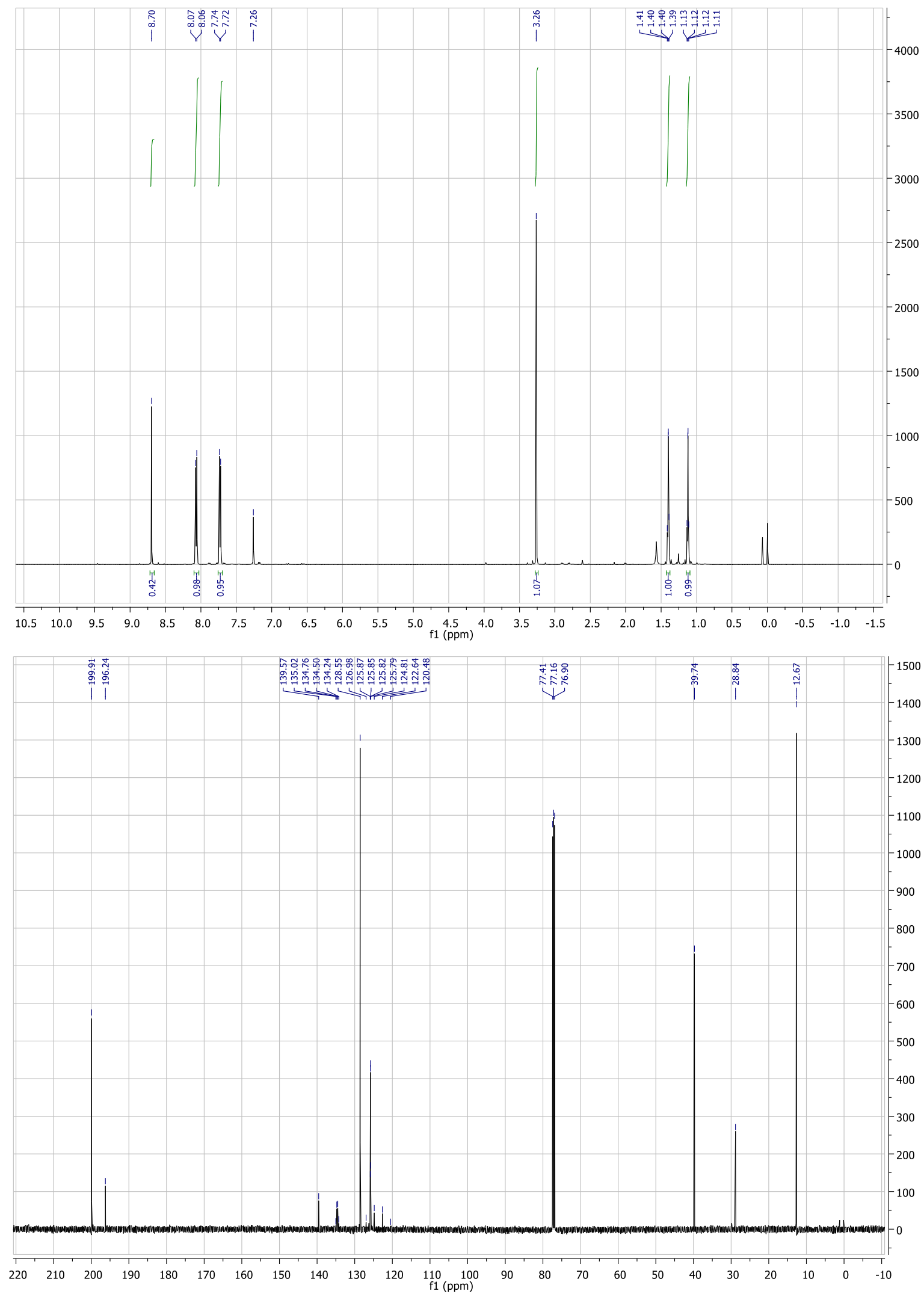


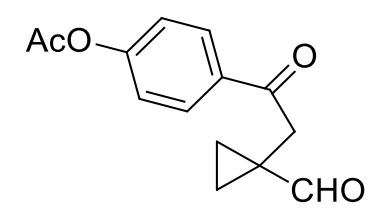

$4 m$
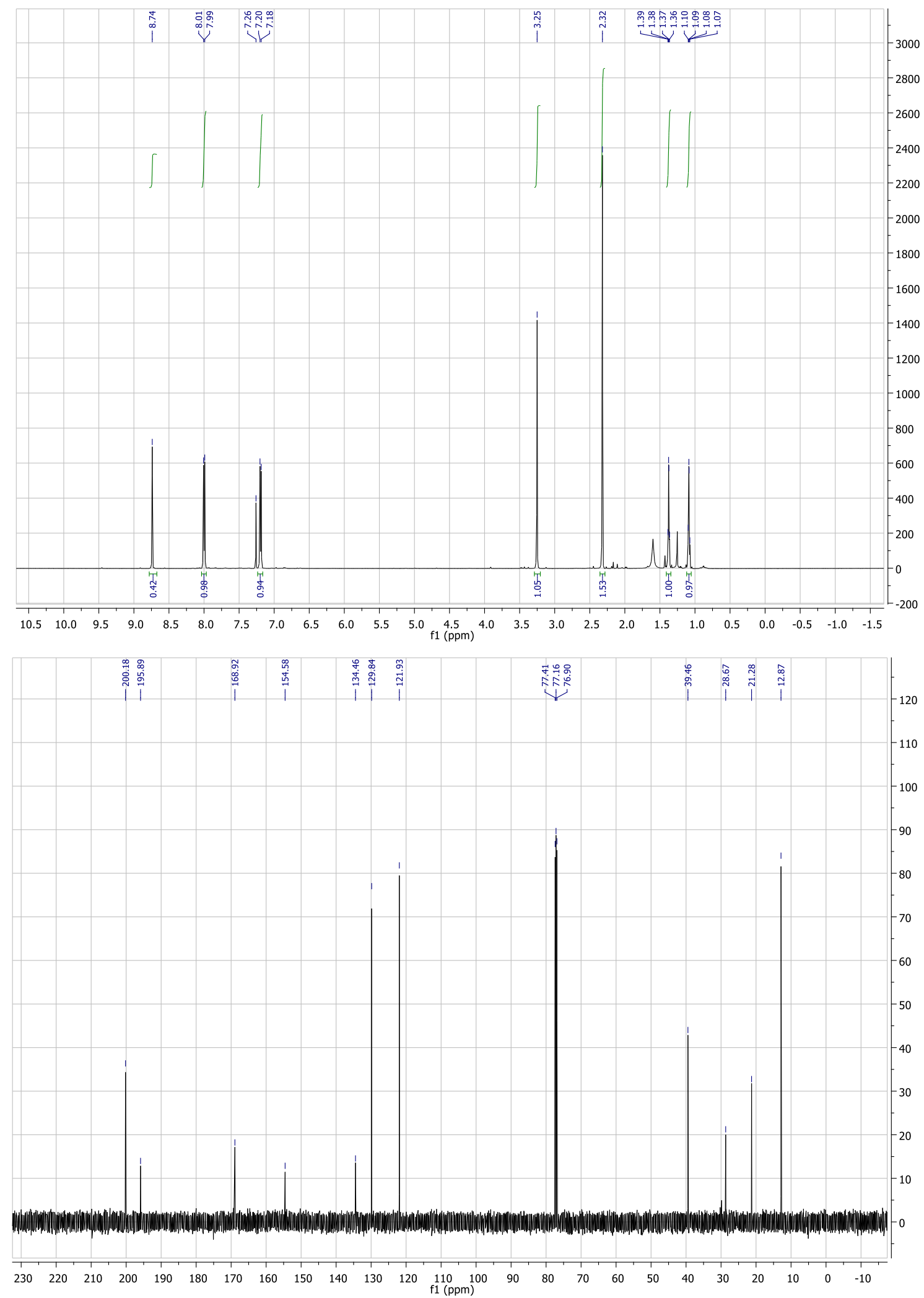


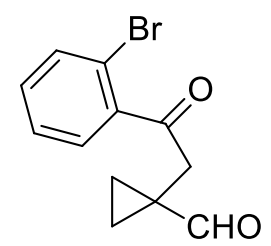

$4 n$
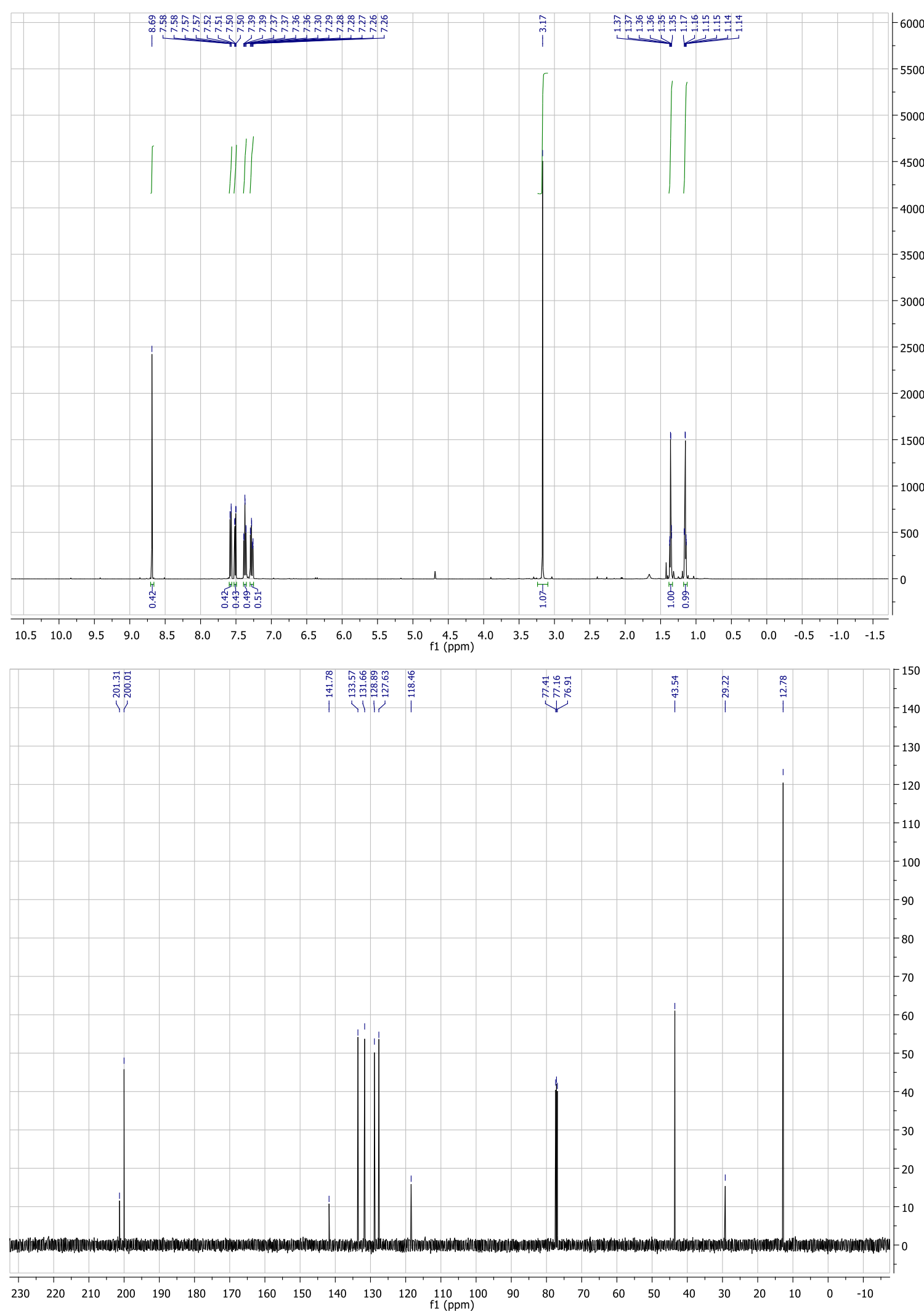


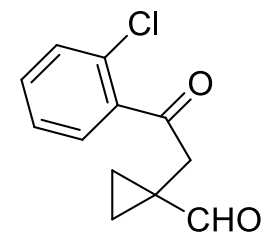

4o
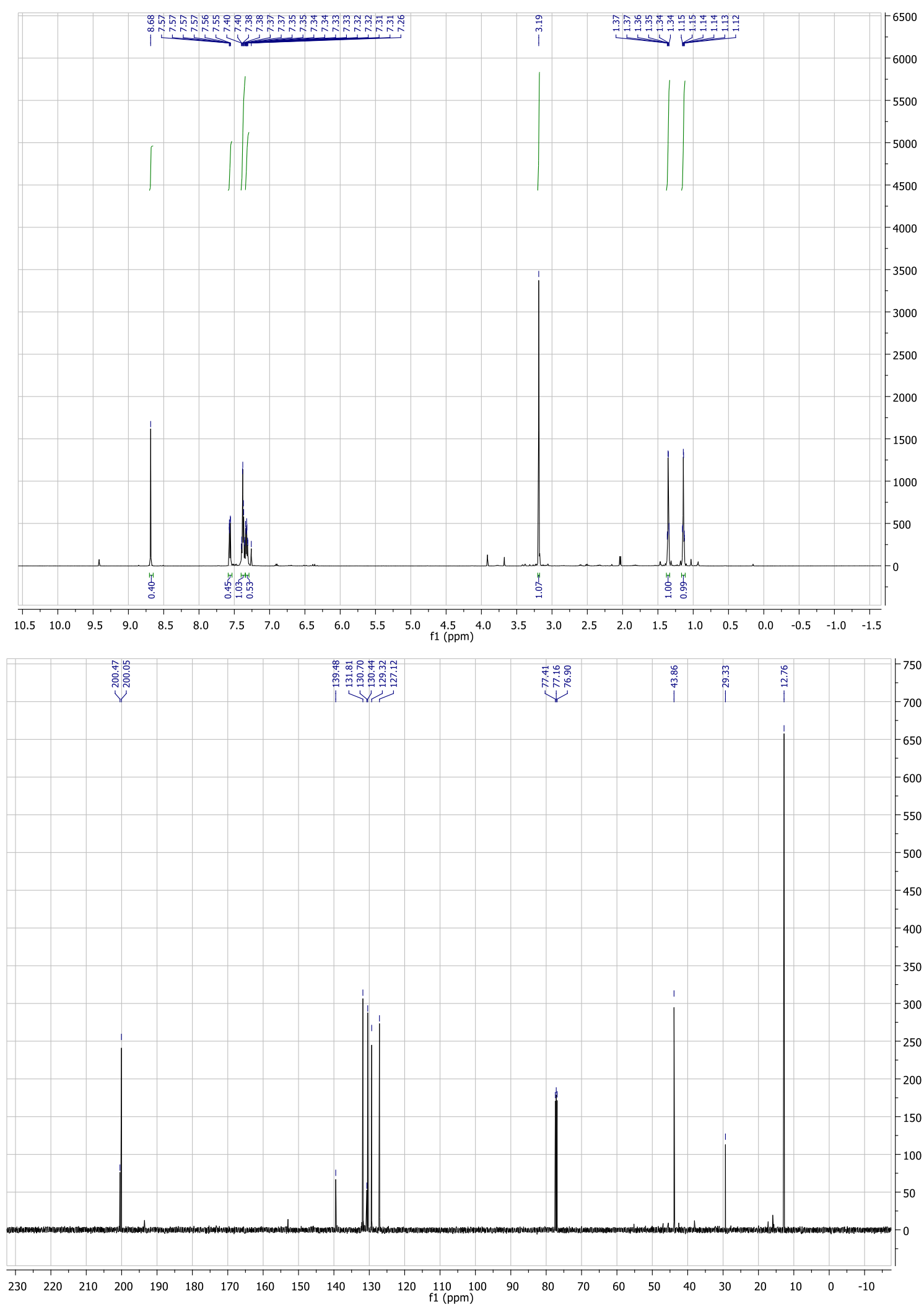


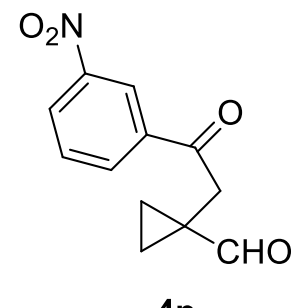

$4 p$
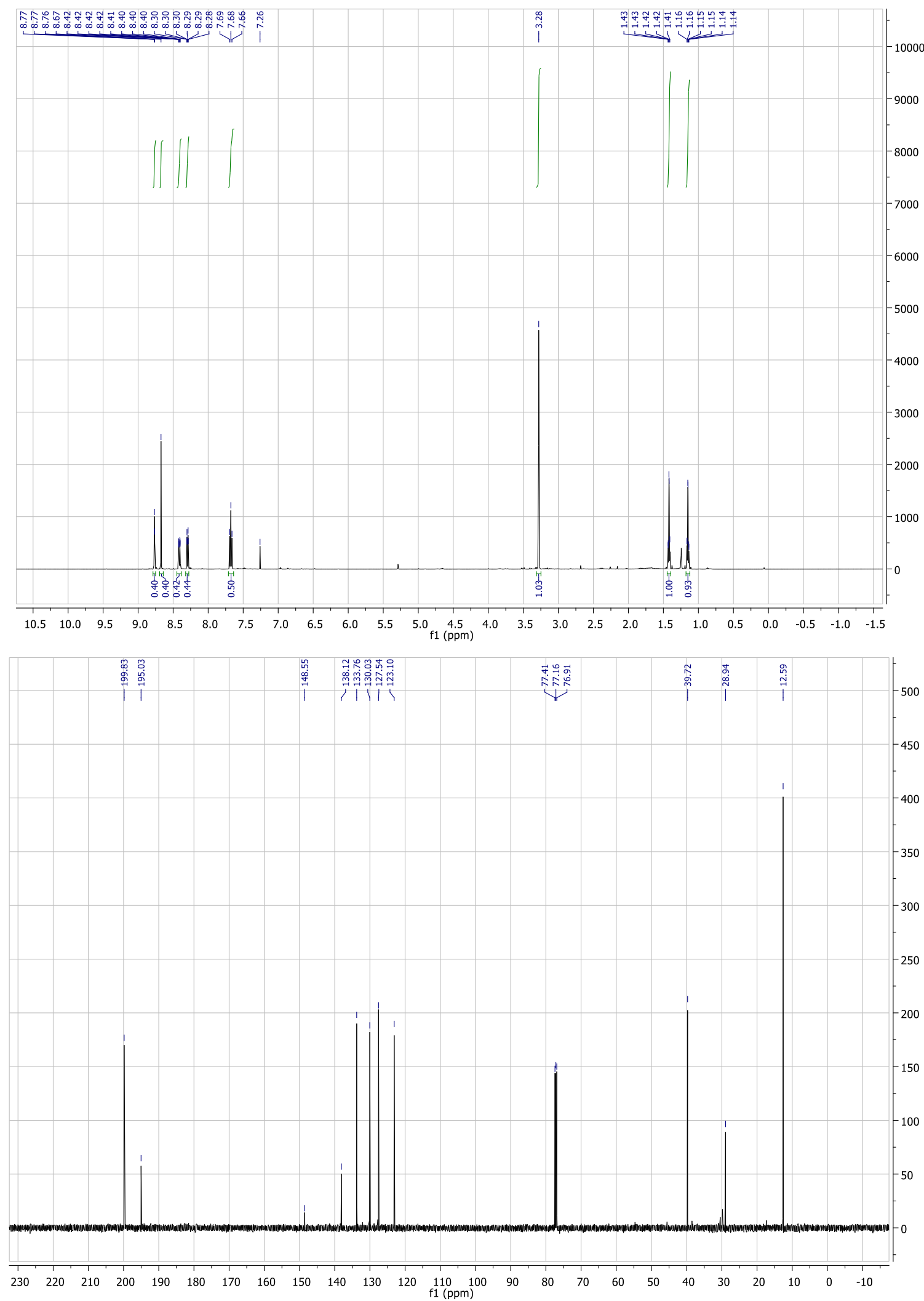


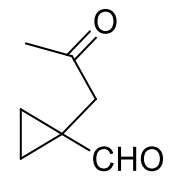

$4 q$

Inseparable mixture of $\mathbf{4 q}$ and unreacted $\alpha$-hydroxycyclobutanone
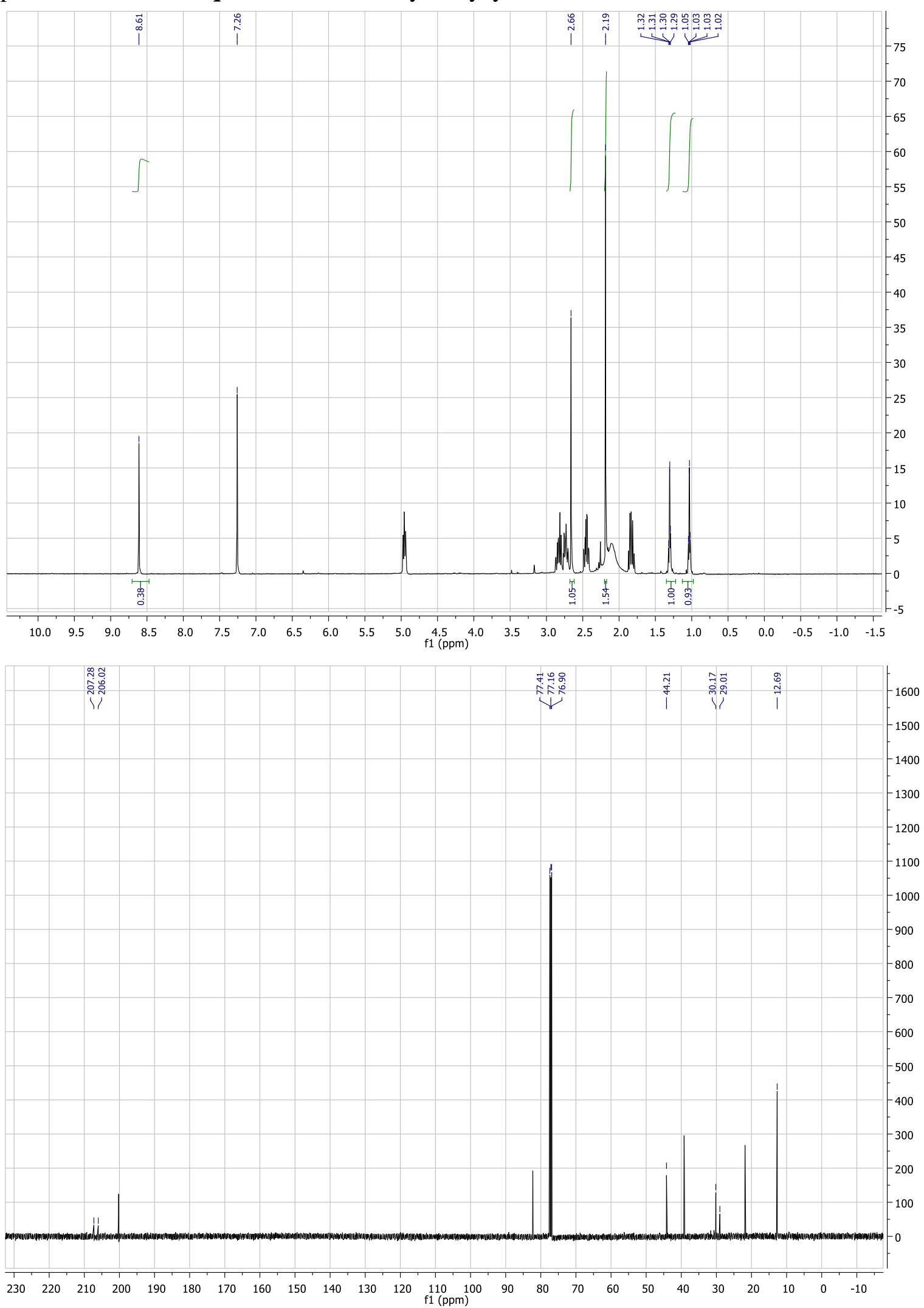


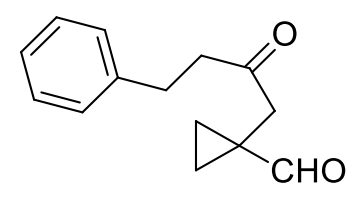

$4 r$
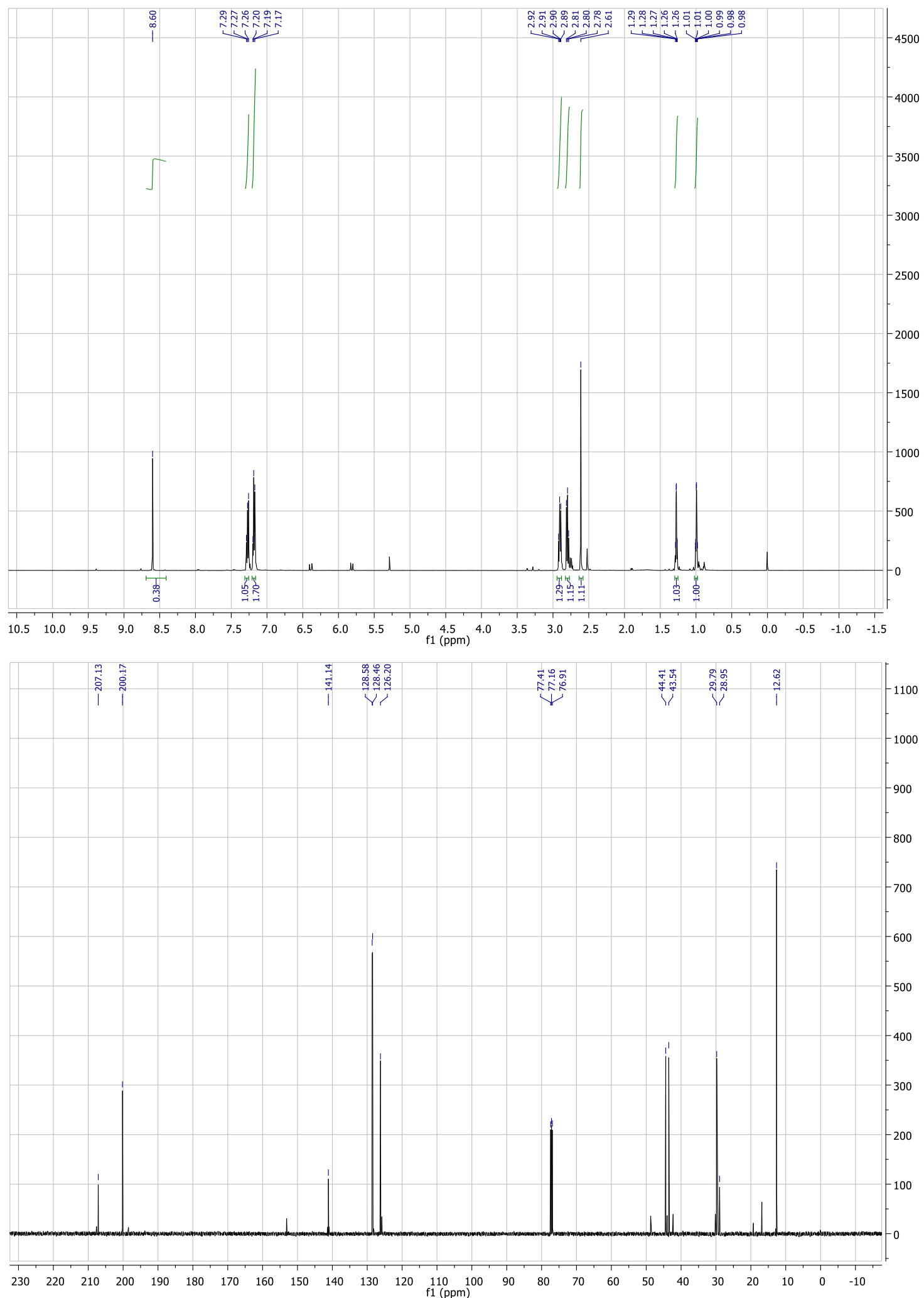


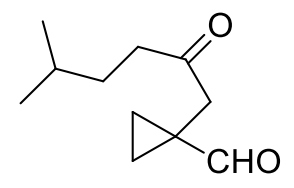

4s
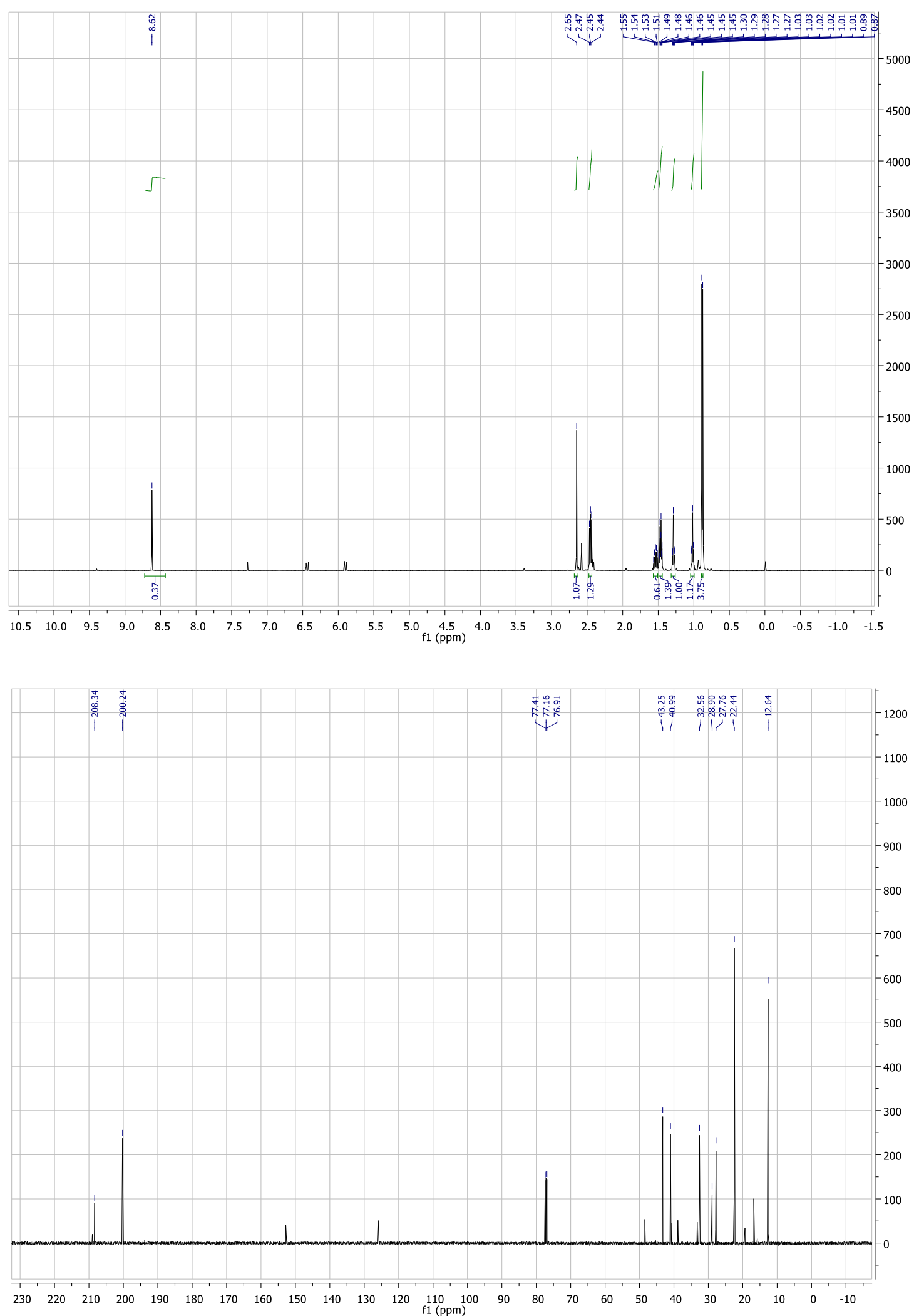


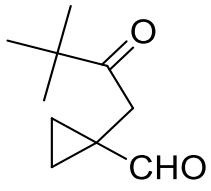

$4 t$
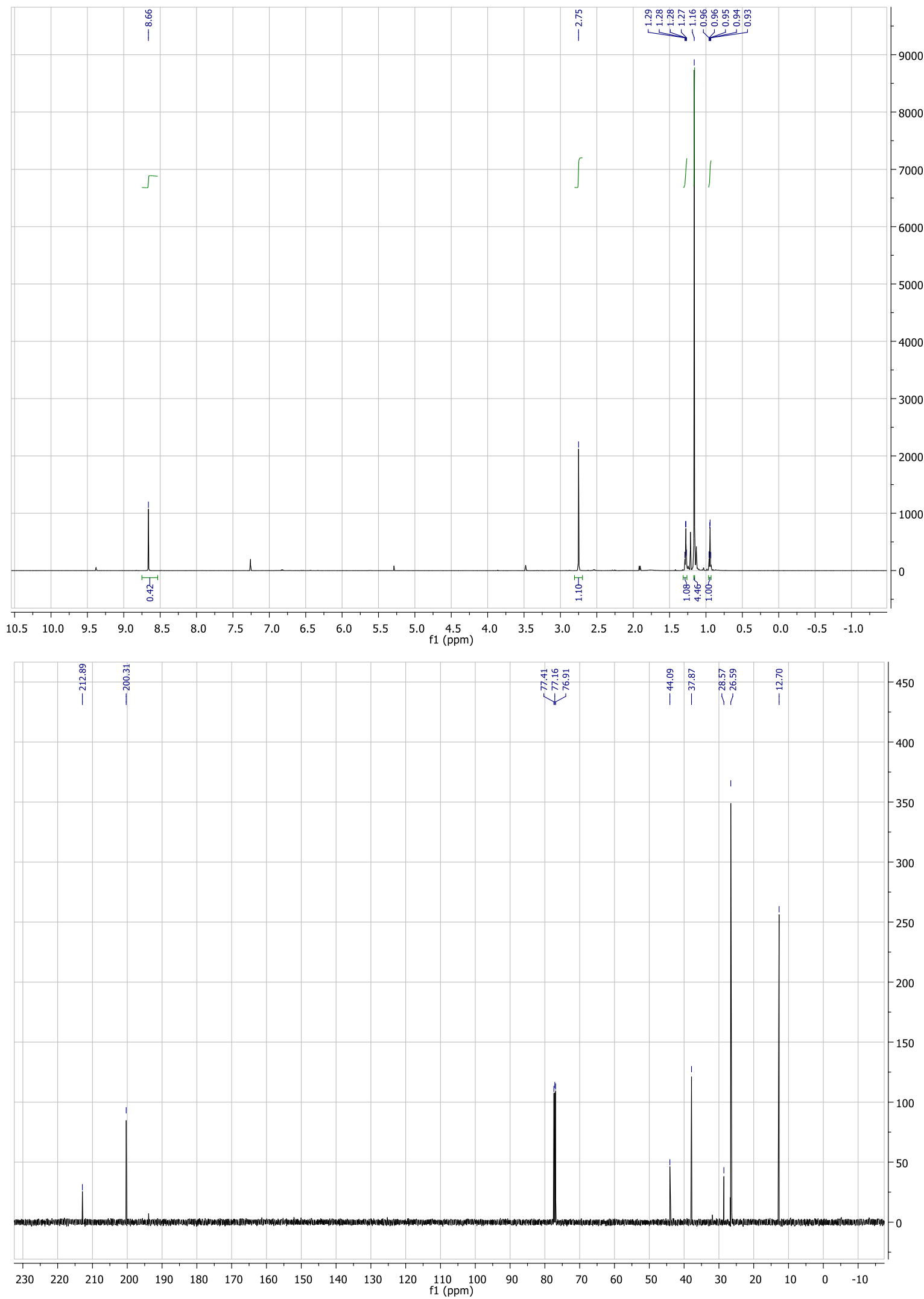
$\Delta \sum_{\mathrm{CHO}}^{\mathrm{O}}$

$4 u$
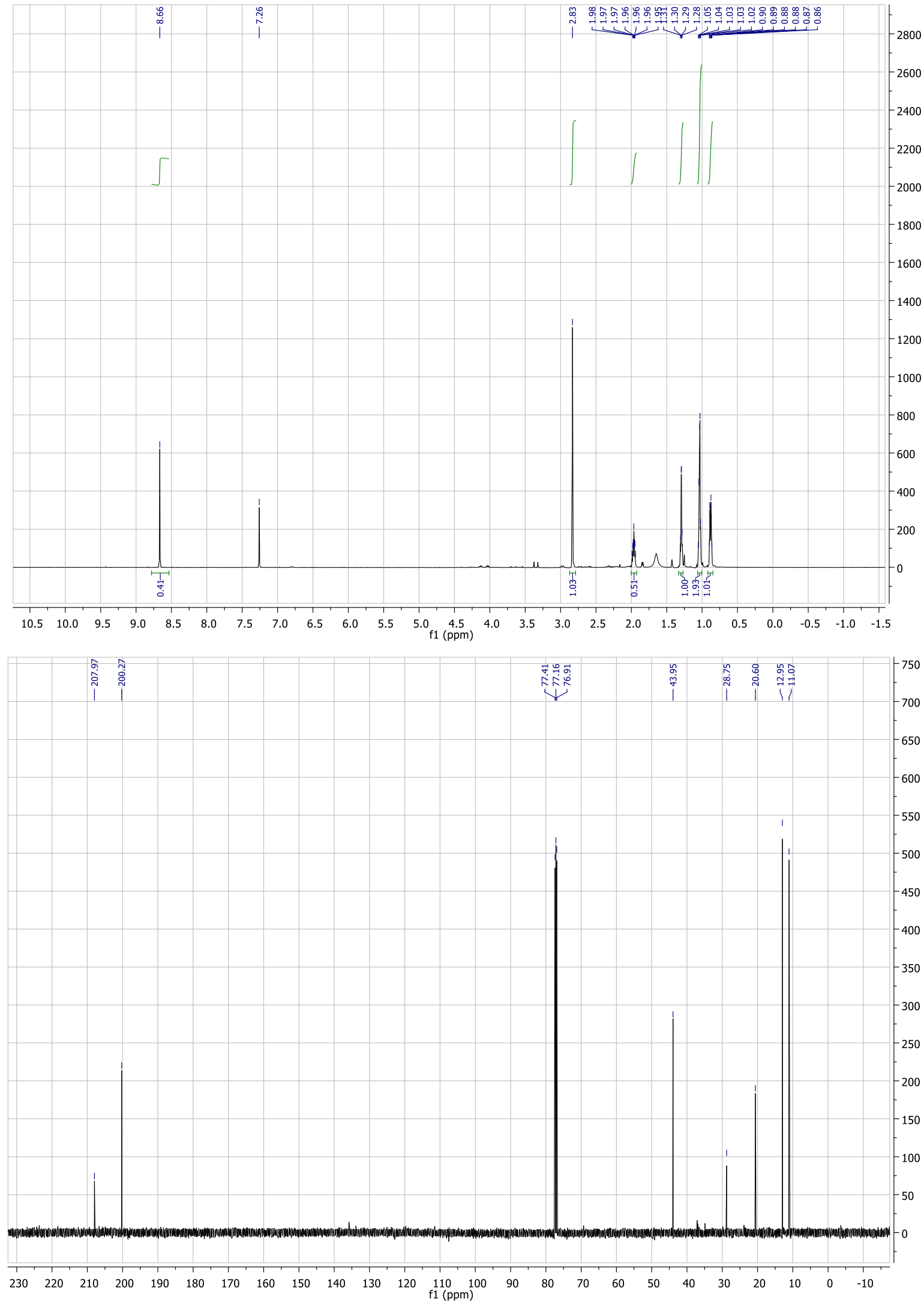


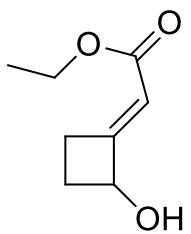

$3 w$

Major isomer
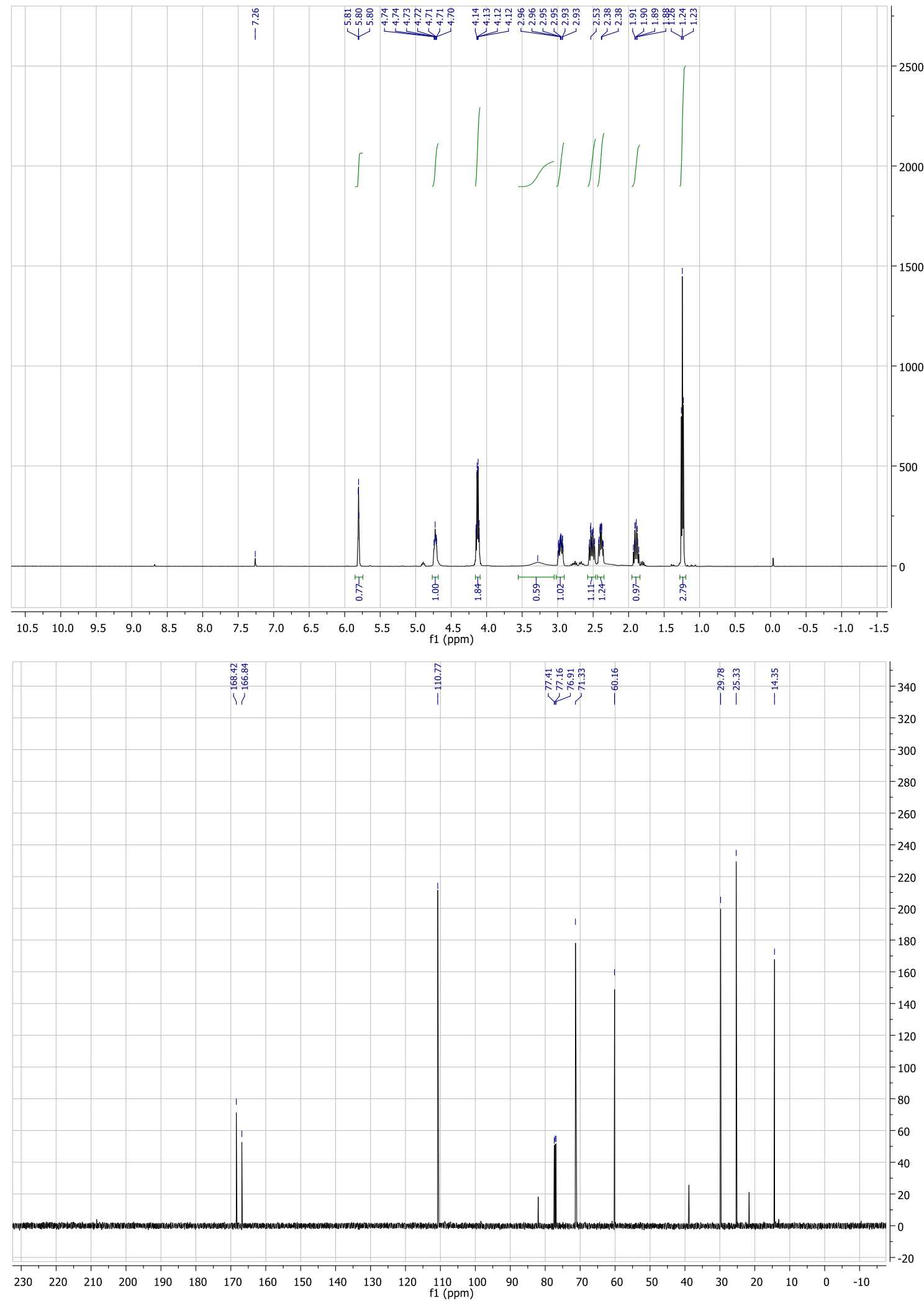


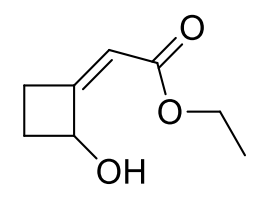

$3 w$

Minor isomer
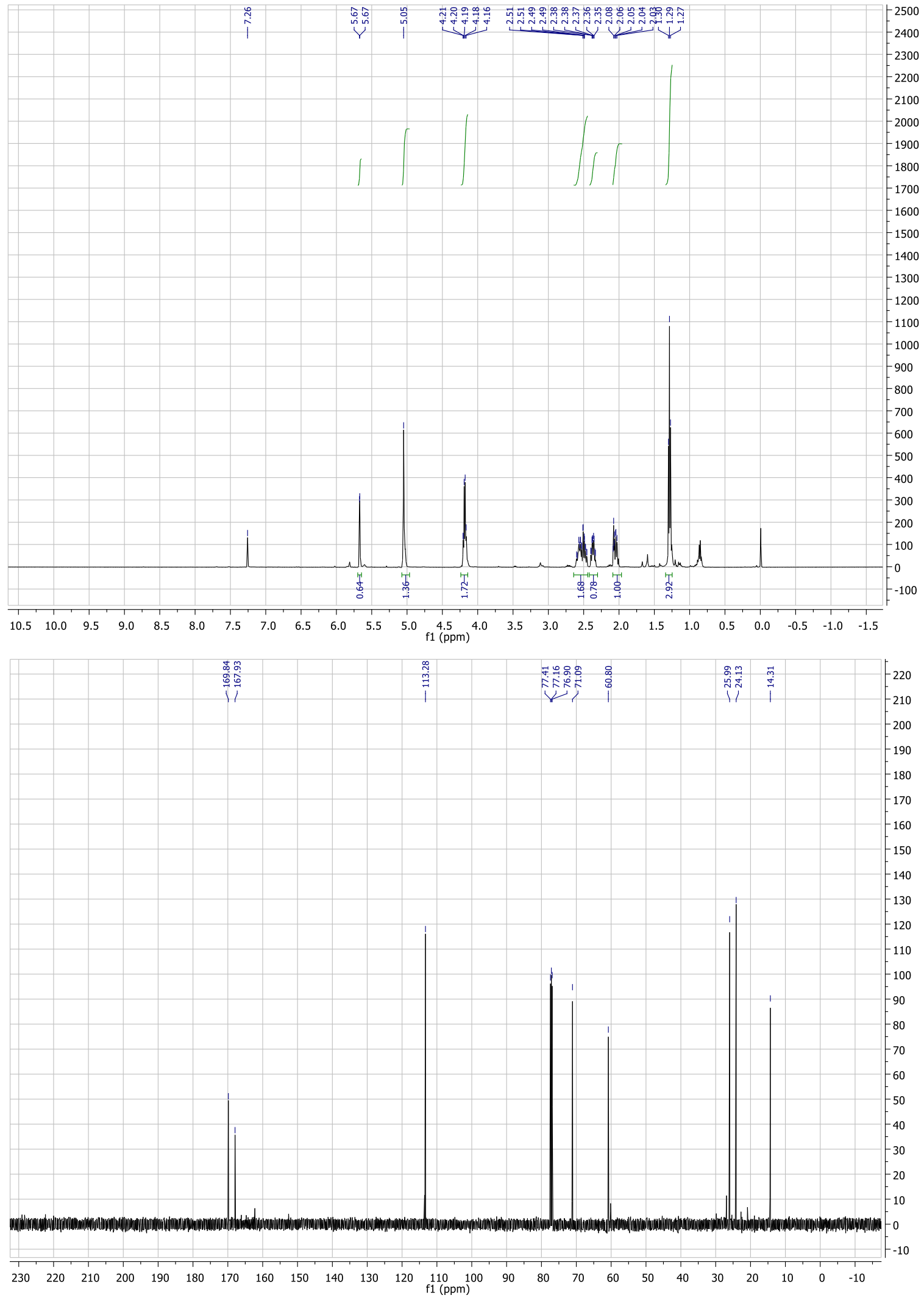


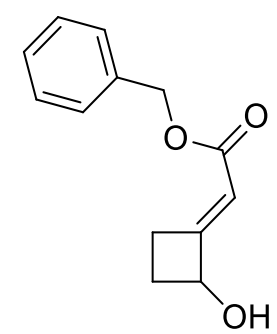

$3 x$

Major isomer
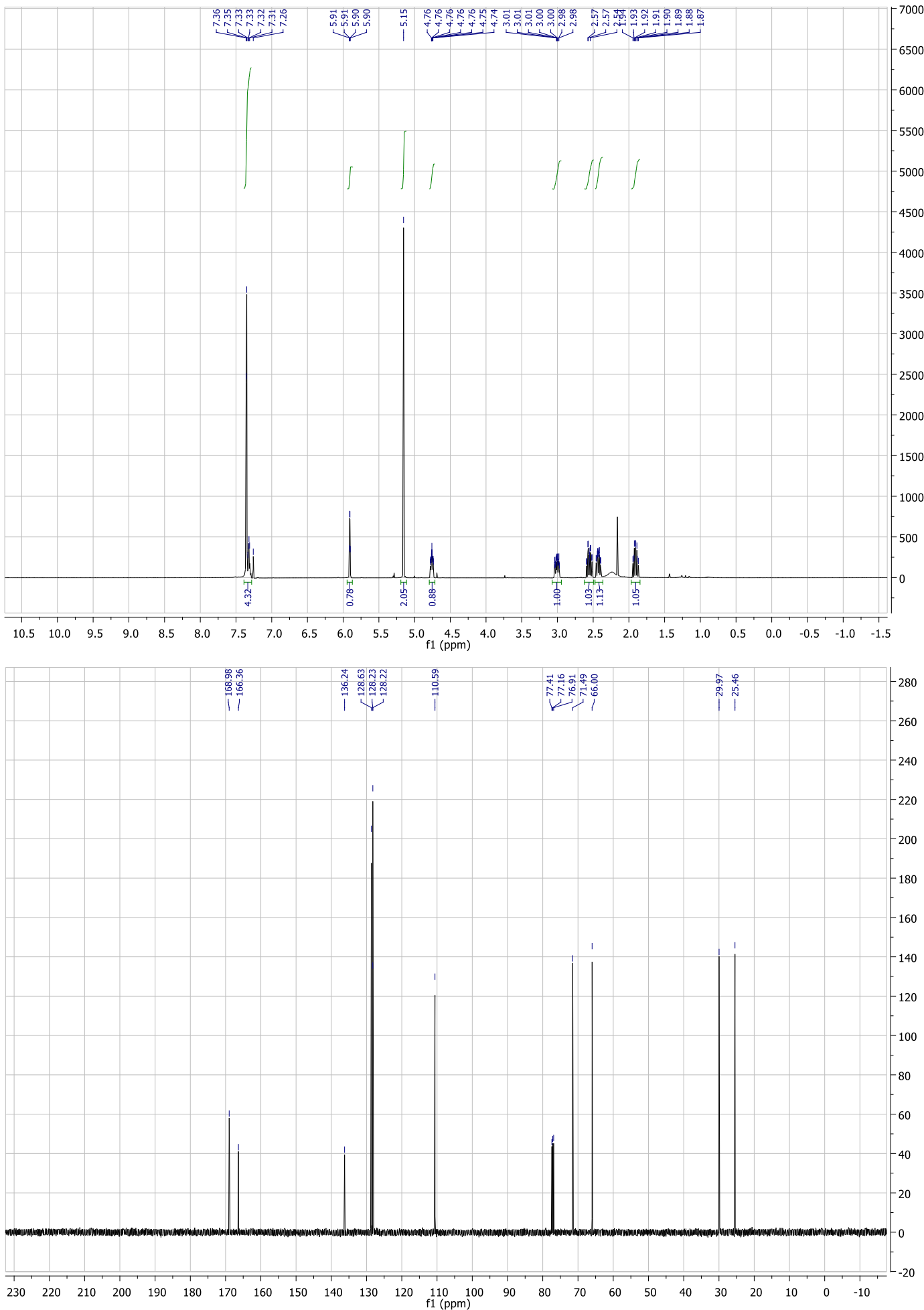


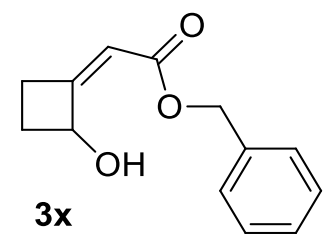

Minor isomer
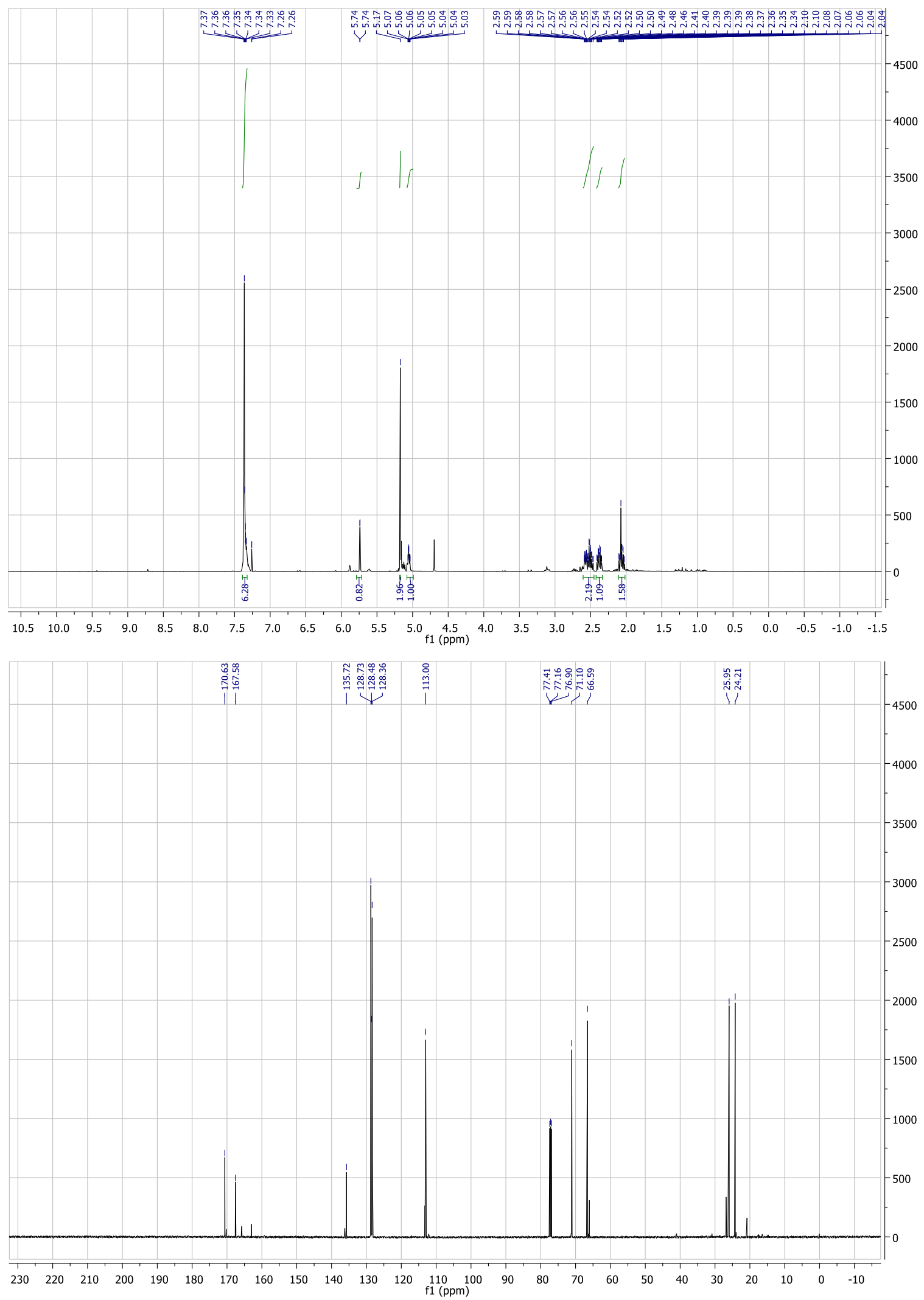


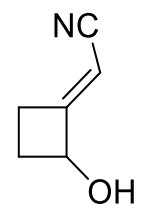

3y

Not separable d.r. 78:22 mixture
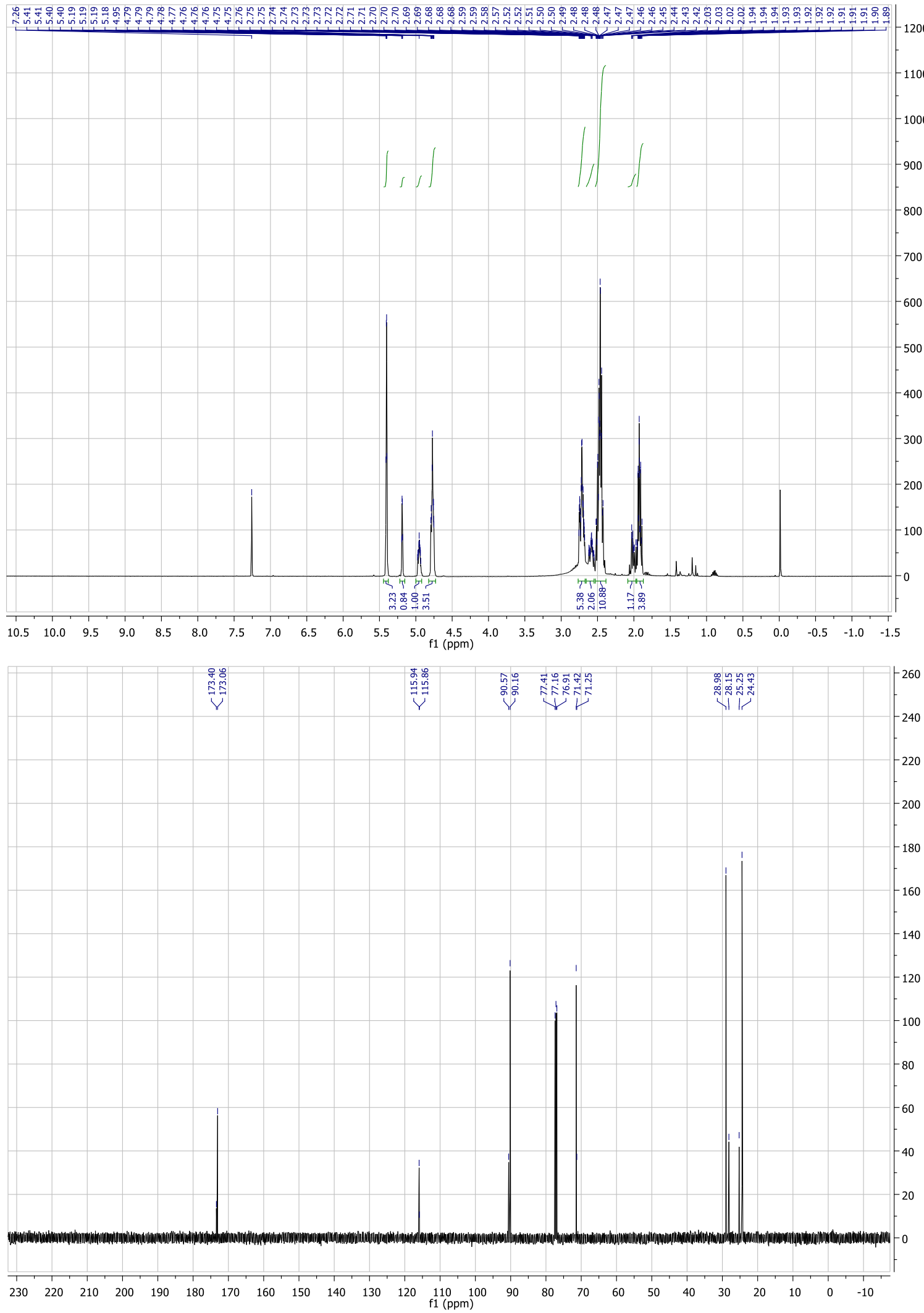


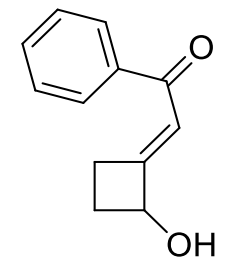

3a

Inseparable mixture of $\mathbf{3 a}$ and triphenylphosphine oxide
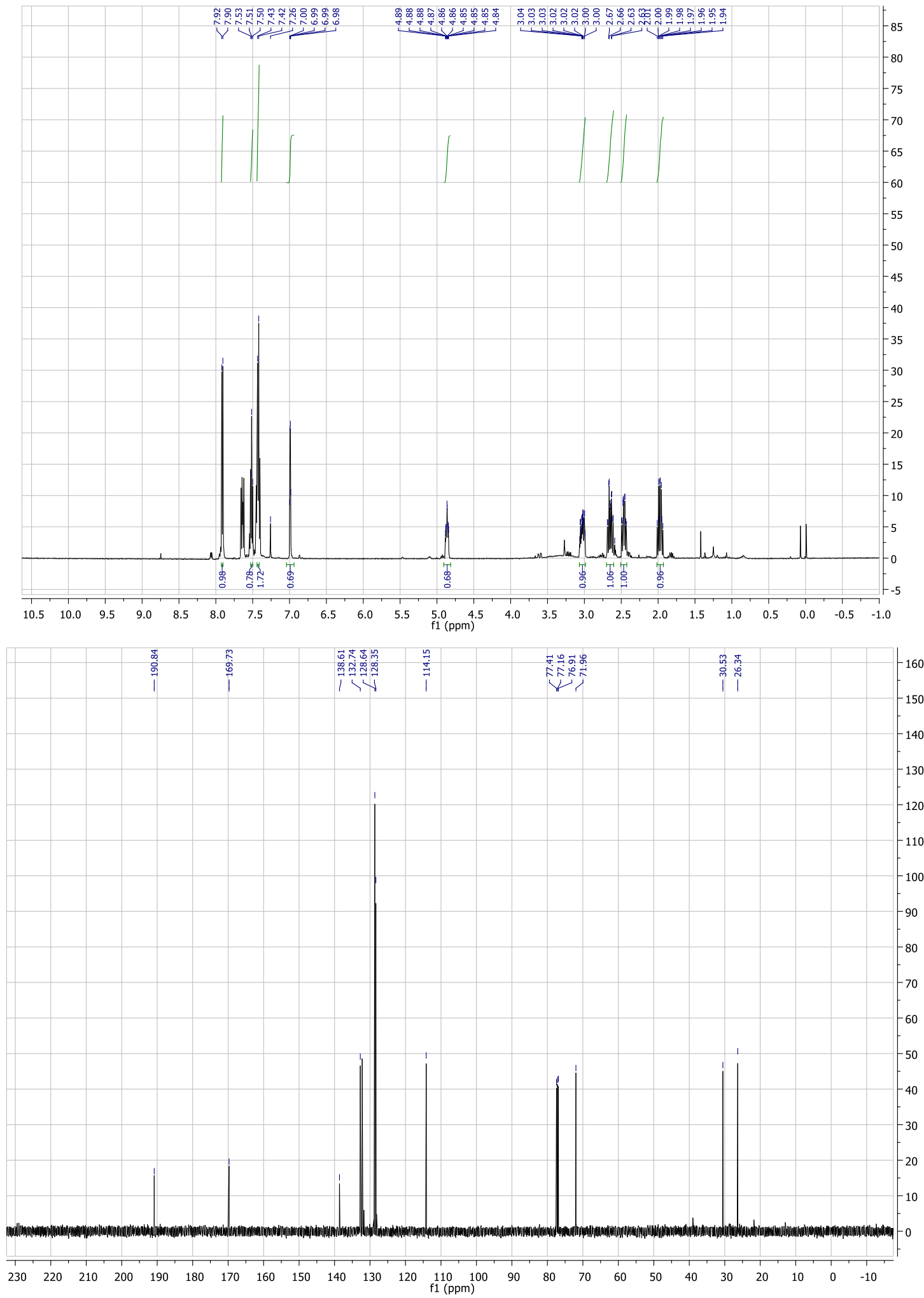

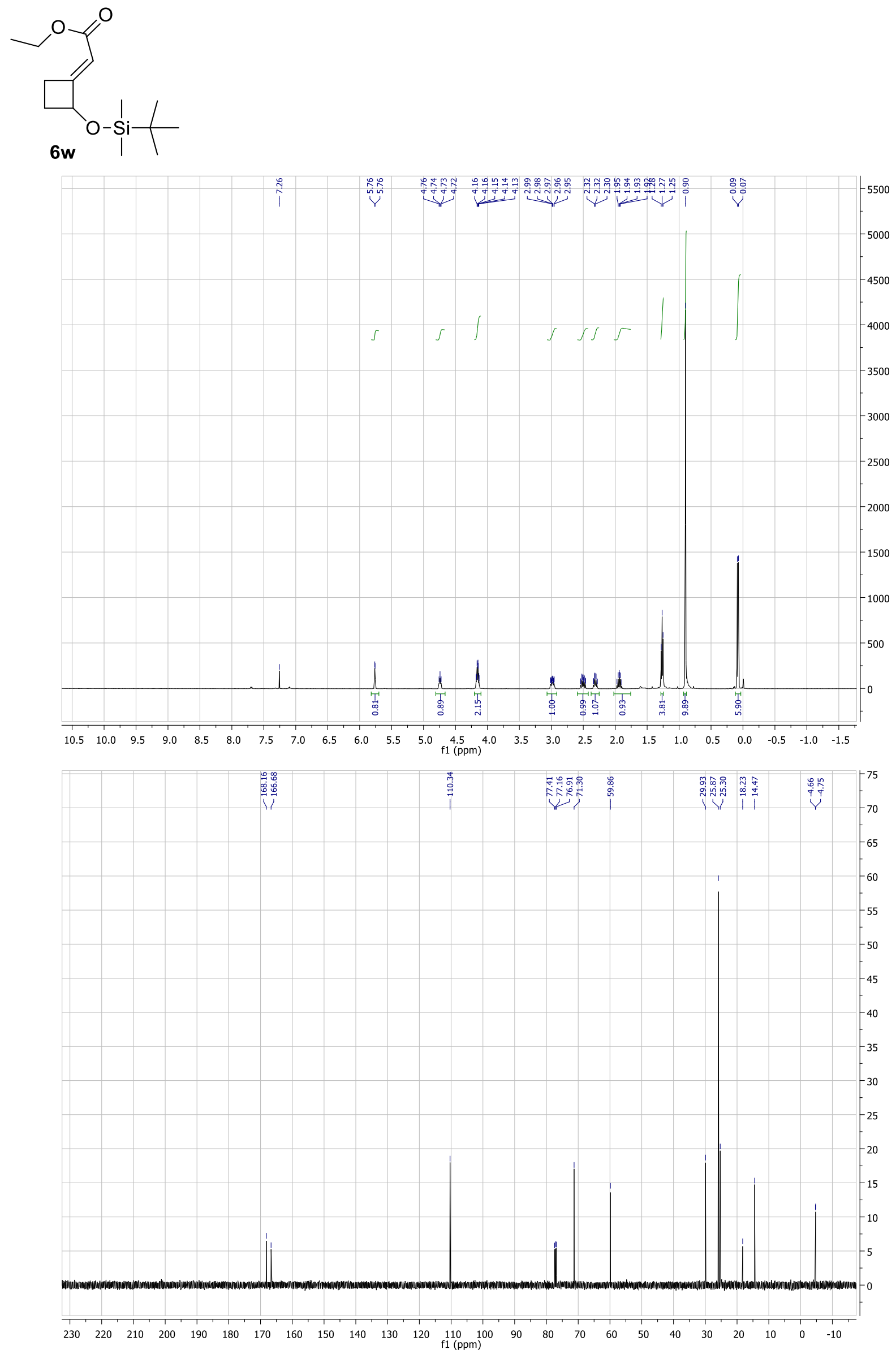

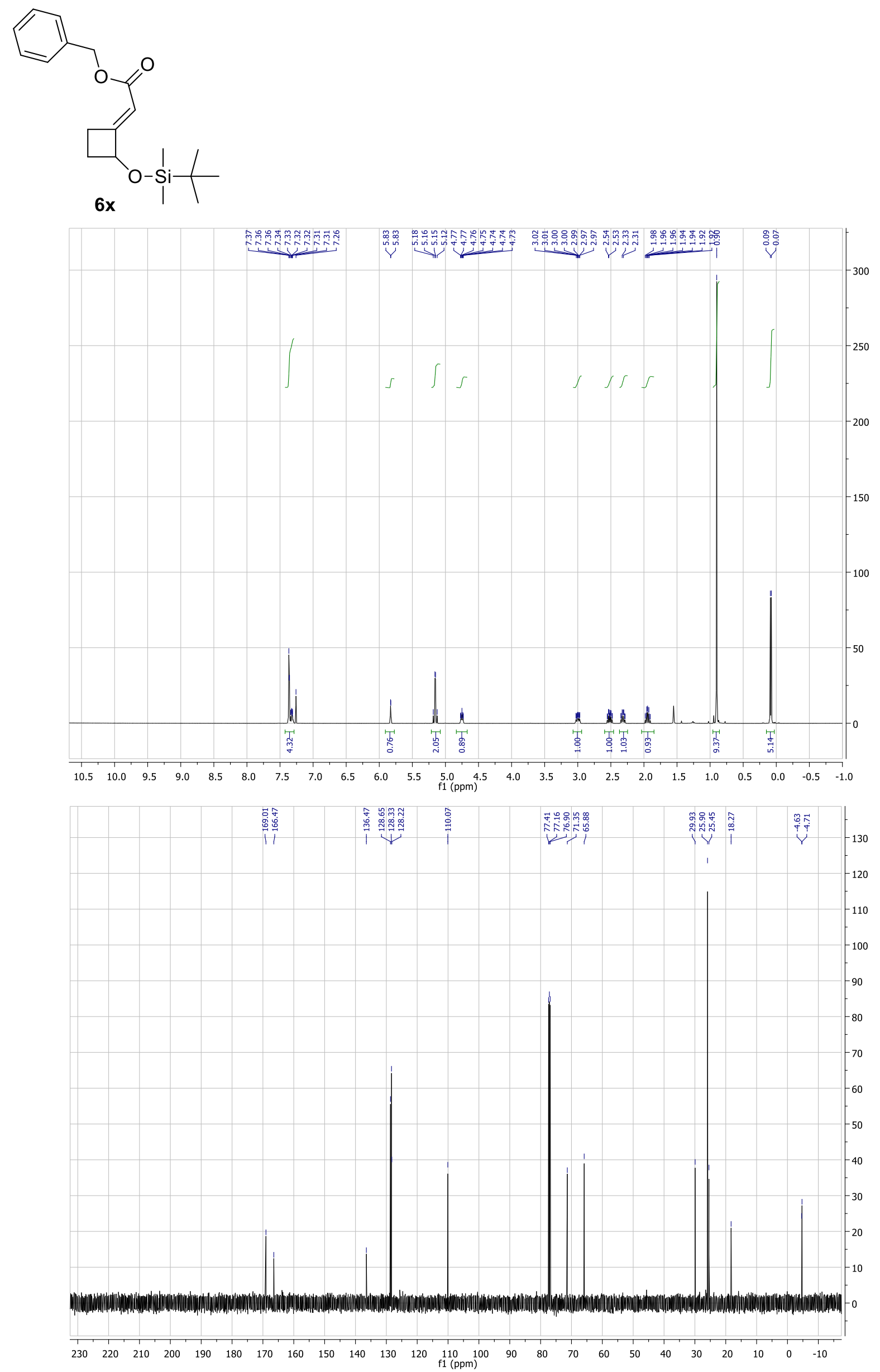


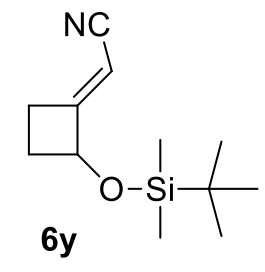

Not separable d.r. 81:19 mixture

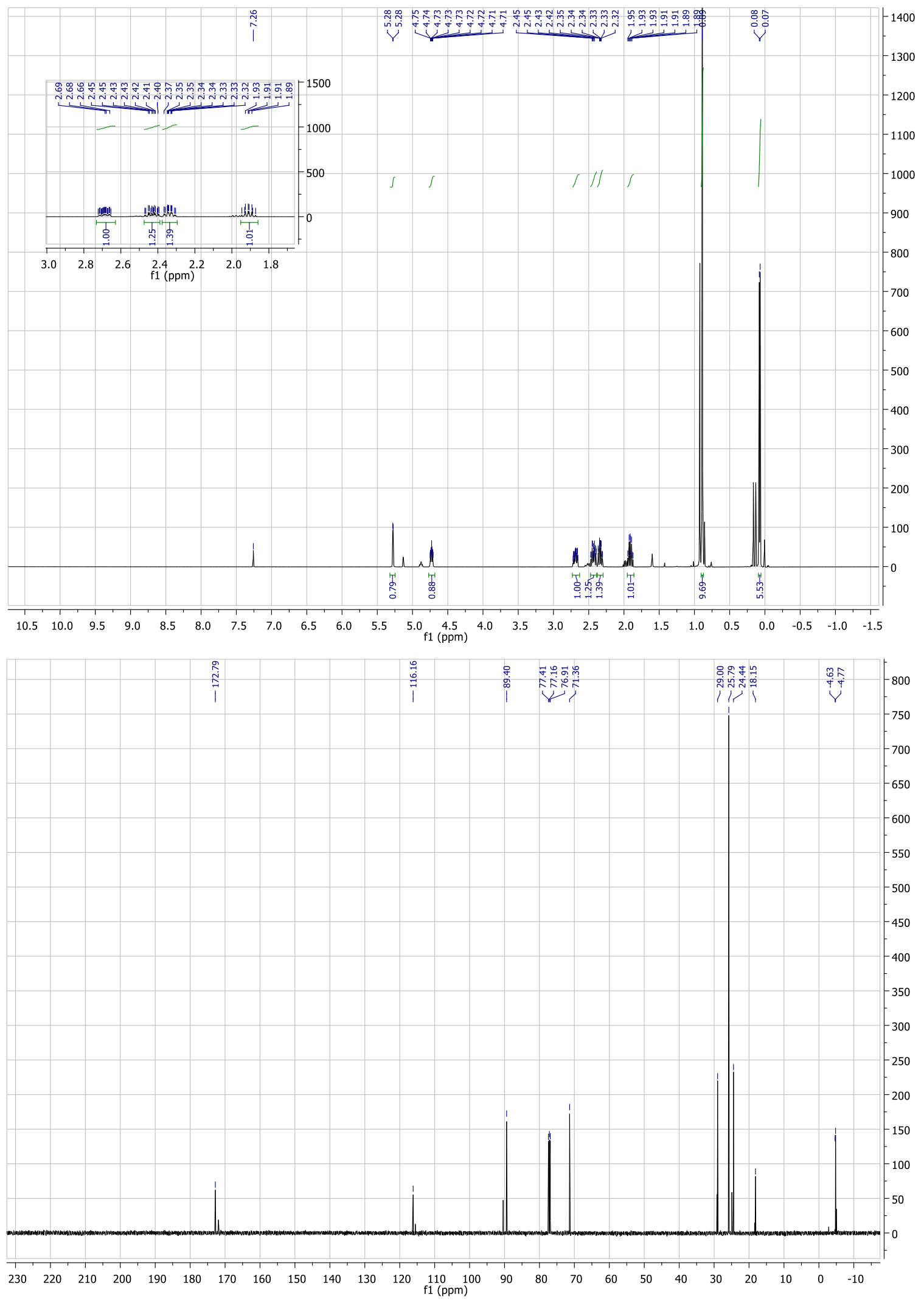




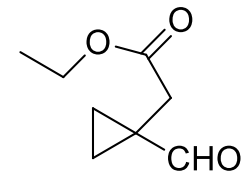

$4 w$
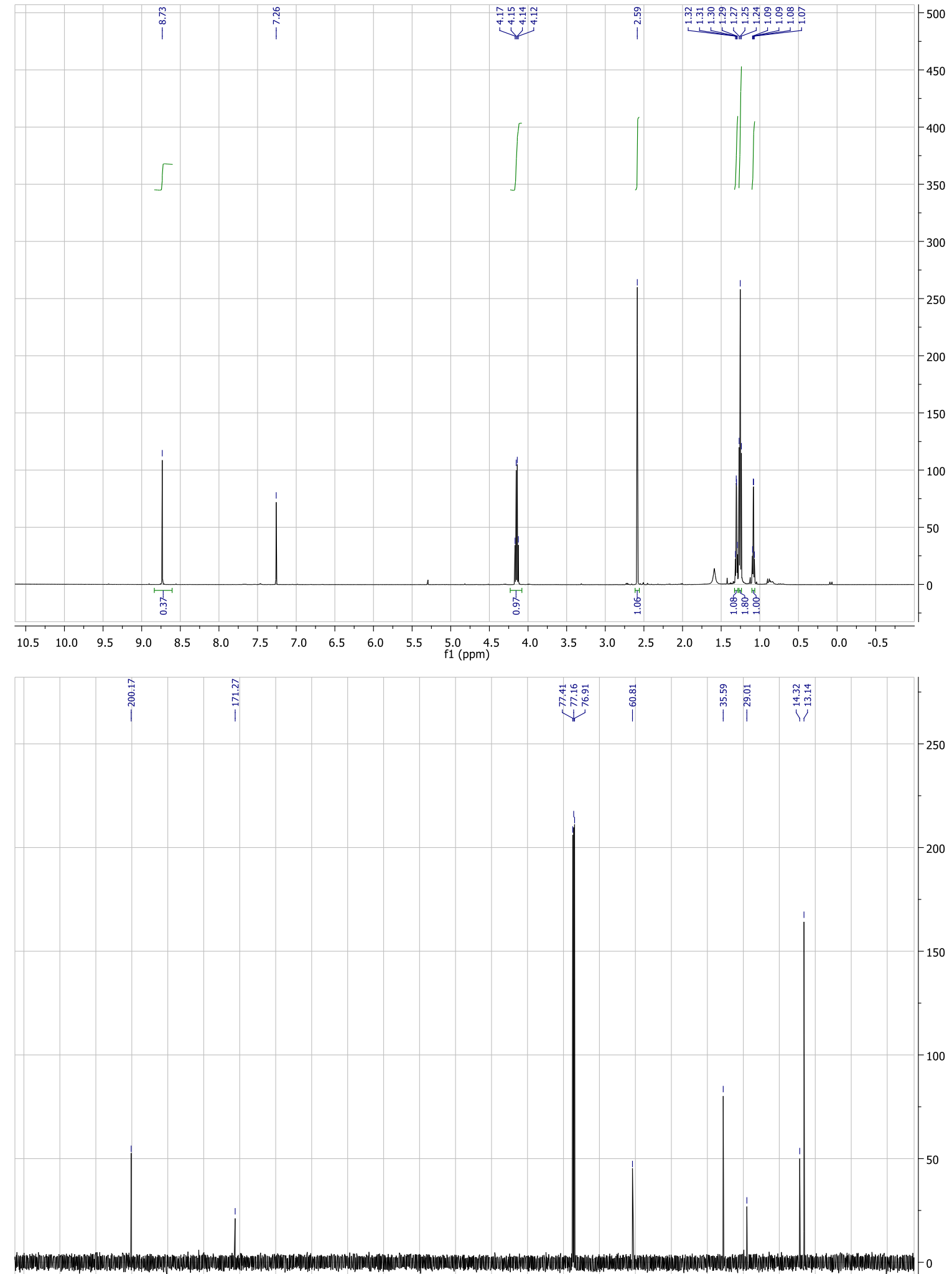

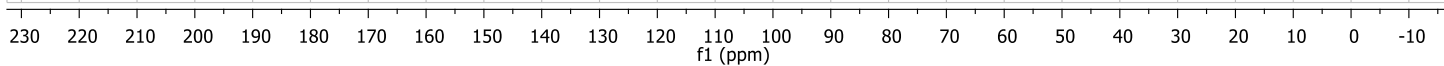



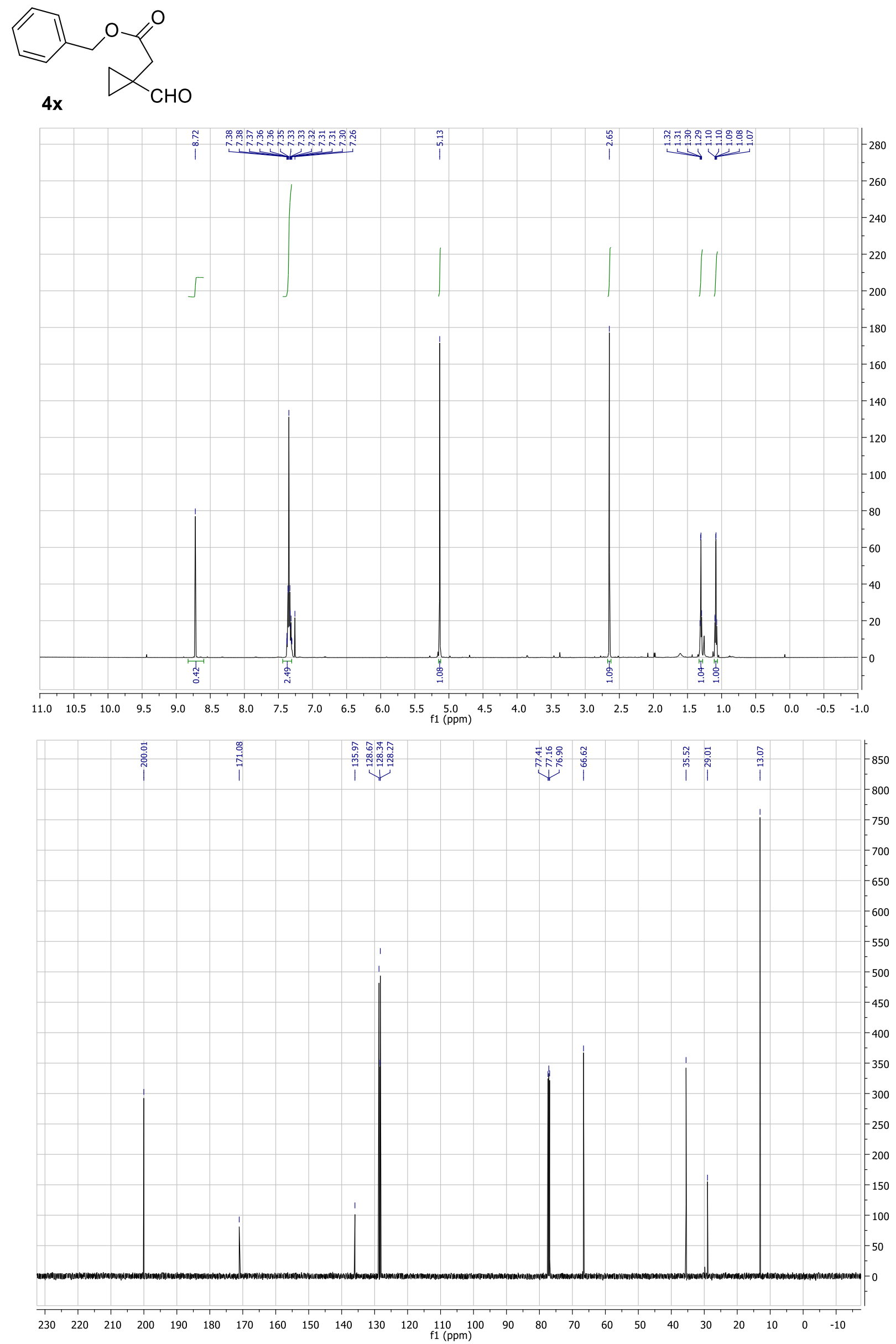


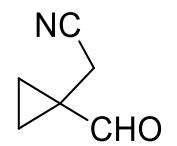

$4 y$
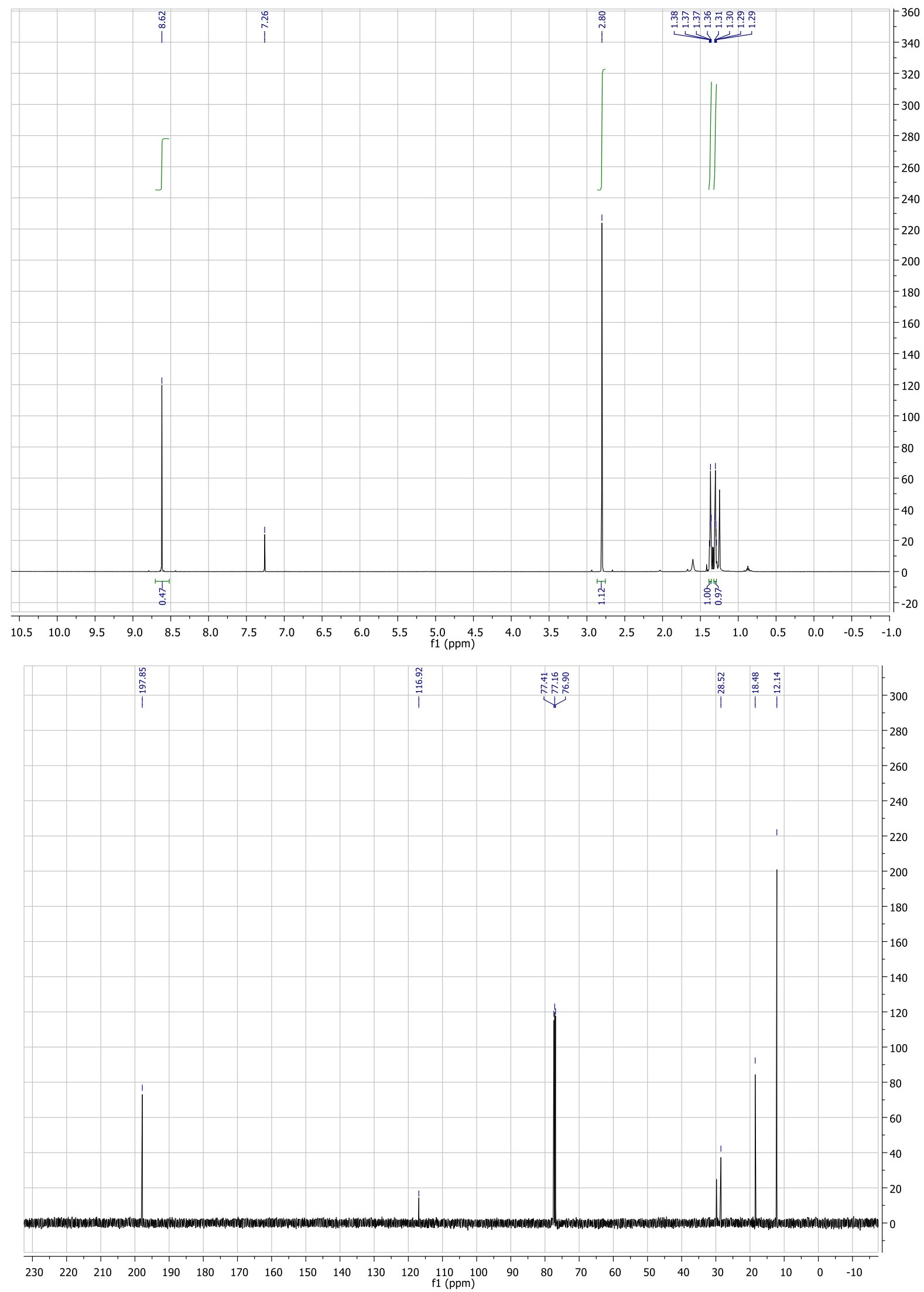


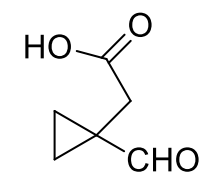

$4 z$
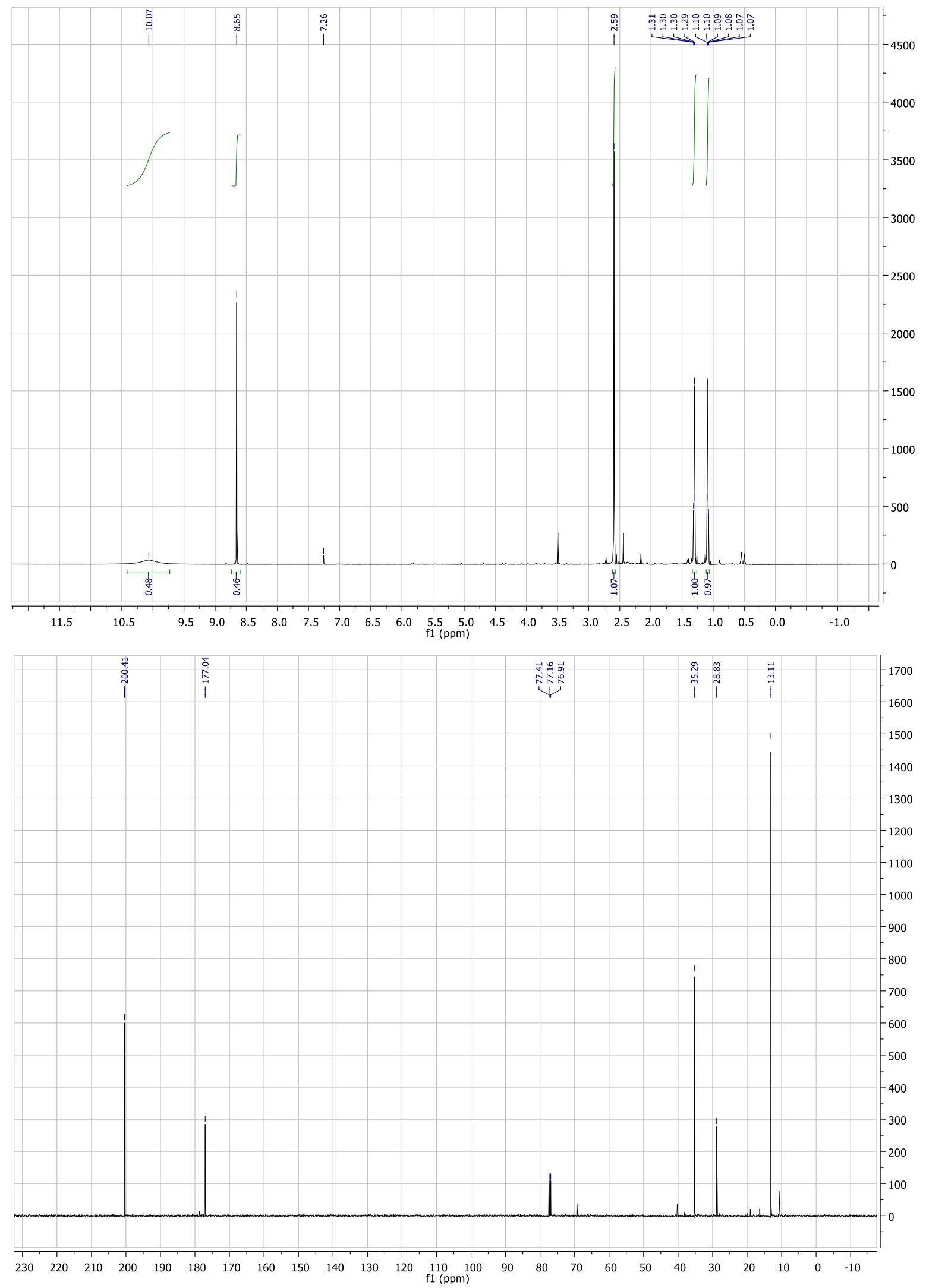


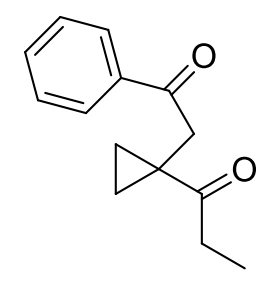

$4 a a$
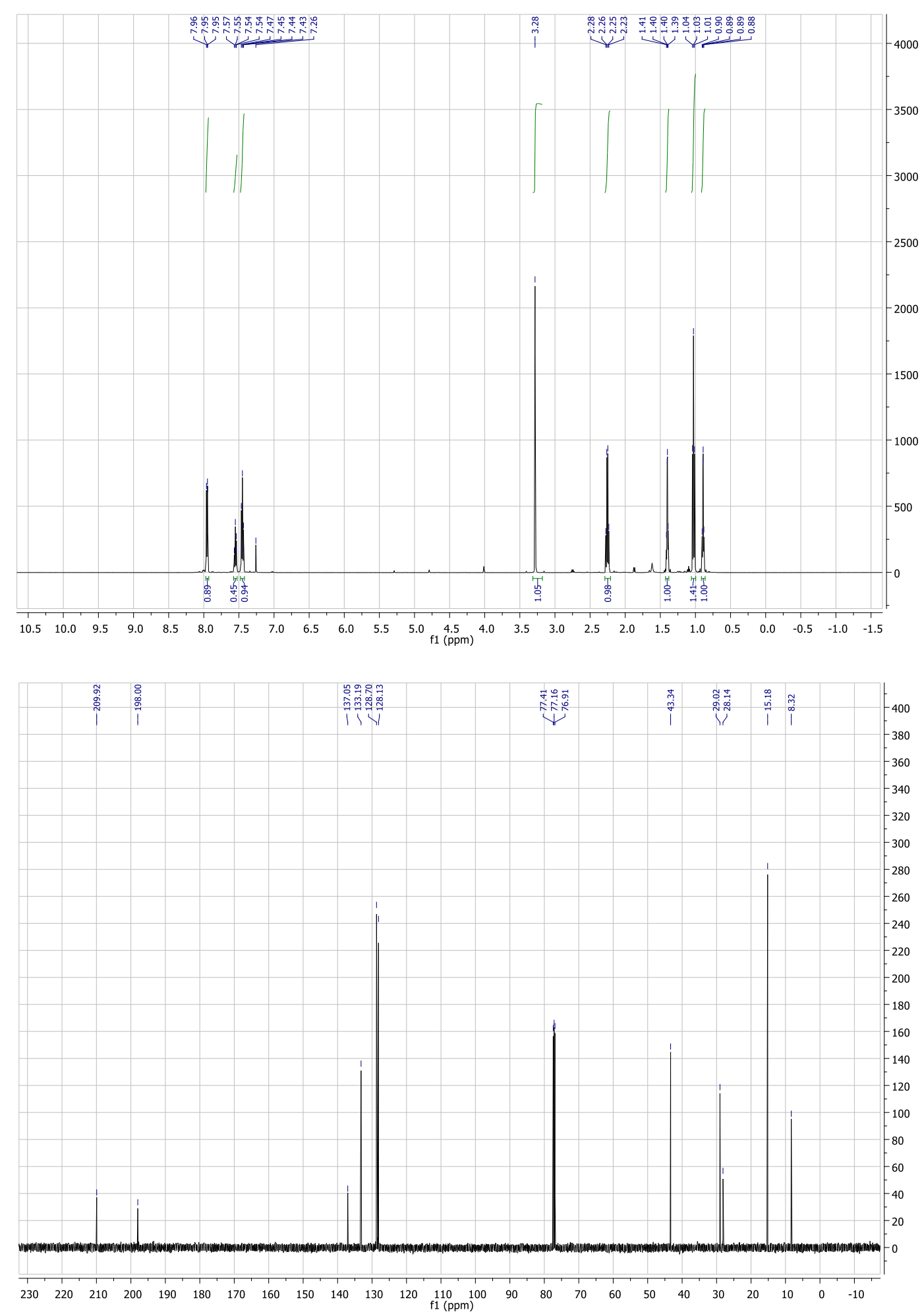


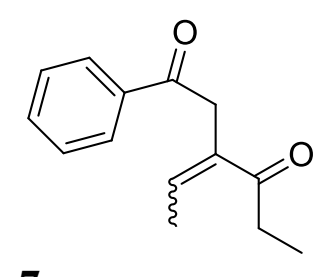

7
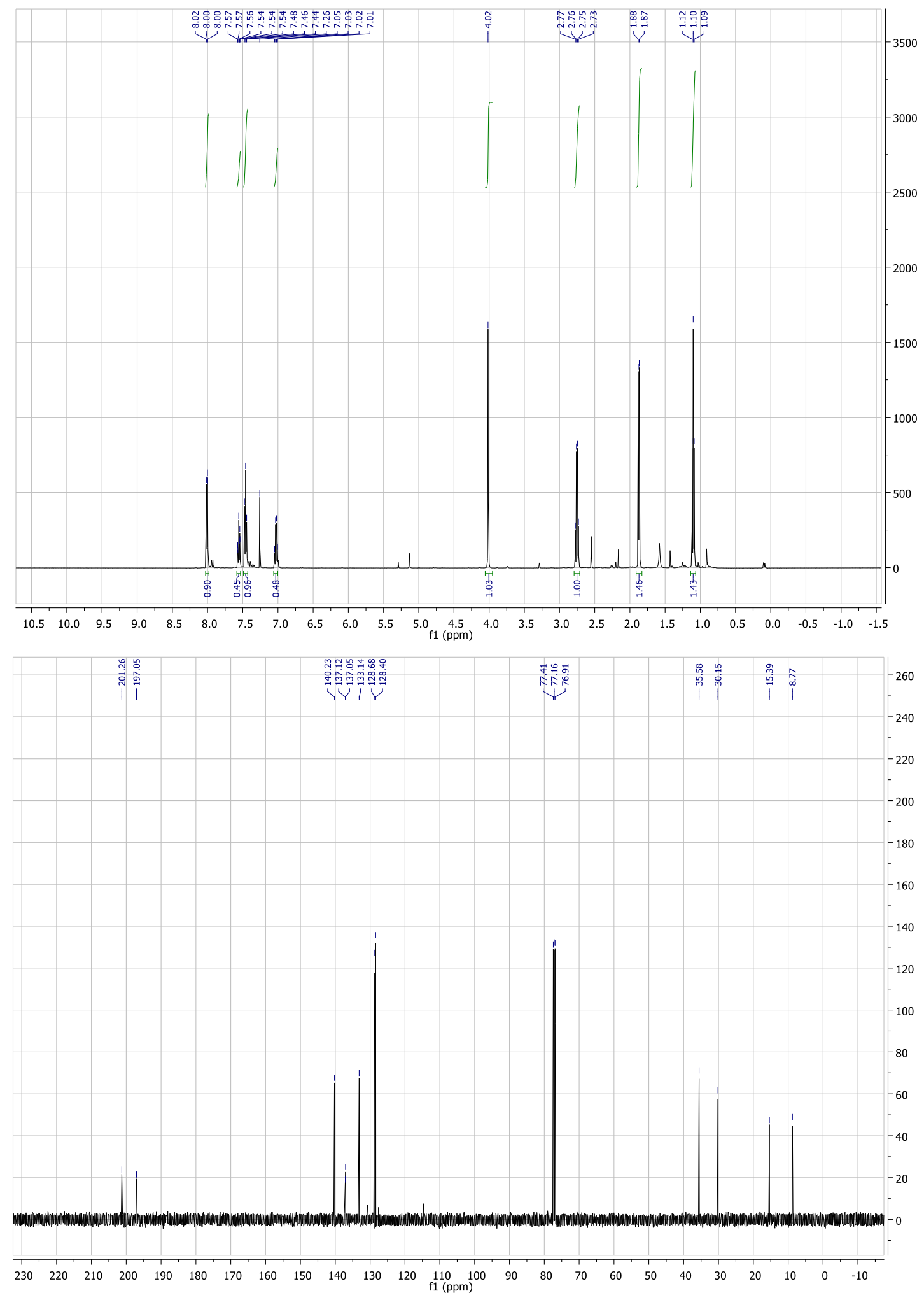
<smiles>O=C(/C=C/C1(CC(=O)c2ccccc2)CC1)c1ccccc1</smiles>
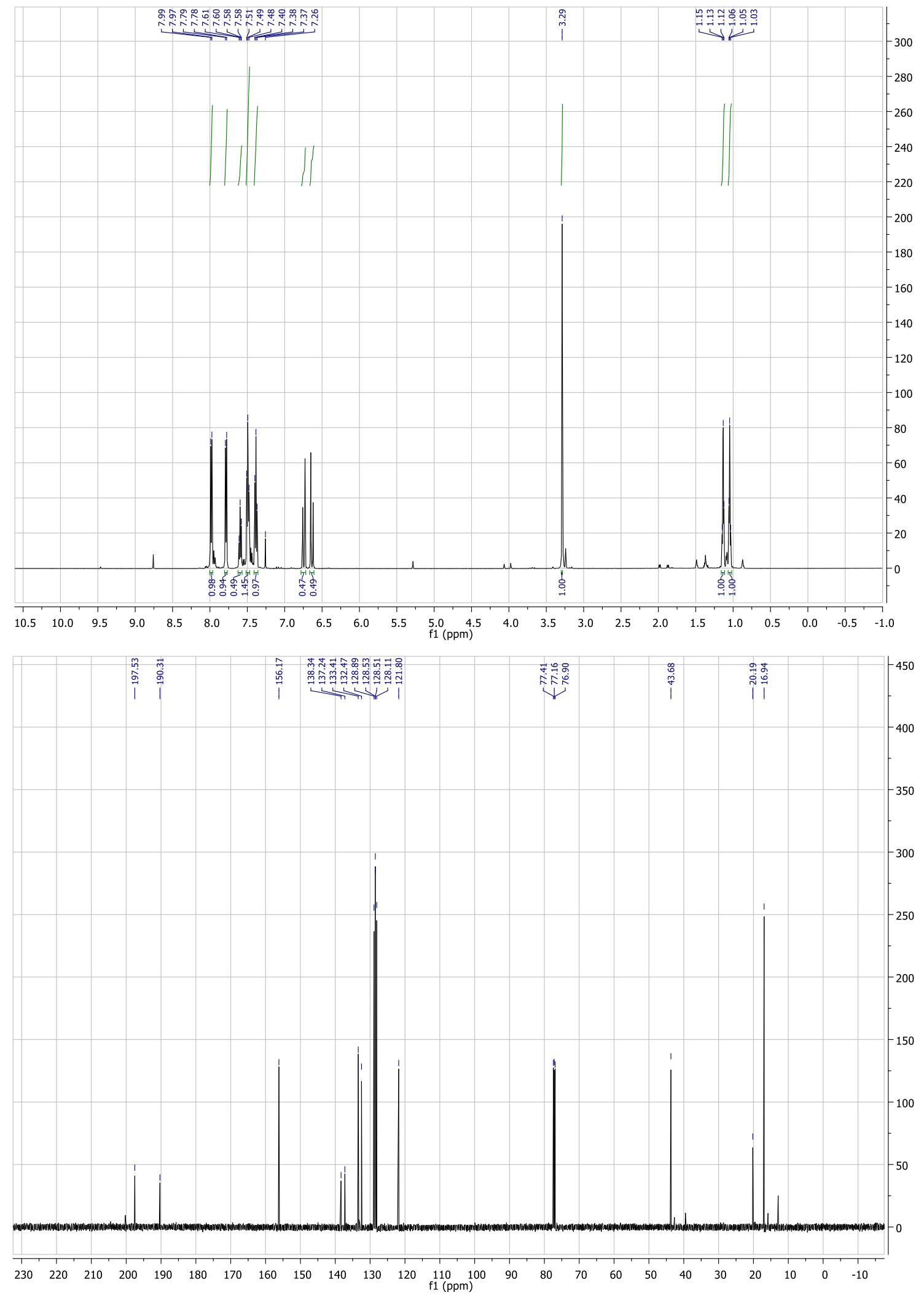

64 
<smiles>Cc1ccc(C(=O)/C=C/C2(CC(=O)c3ccc(C)cc3)CC2)cc1</smiles>
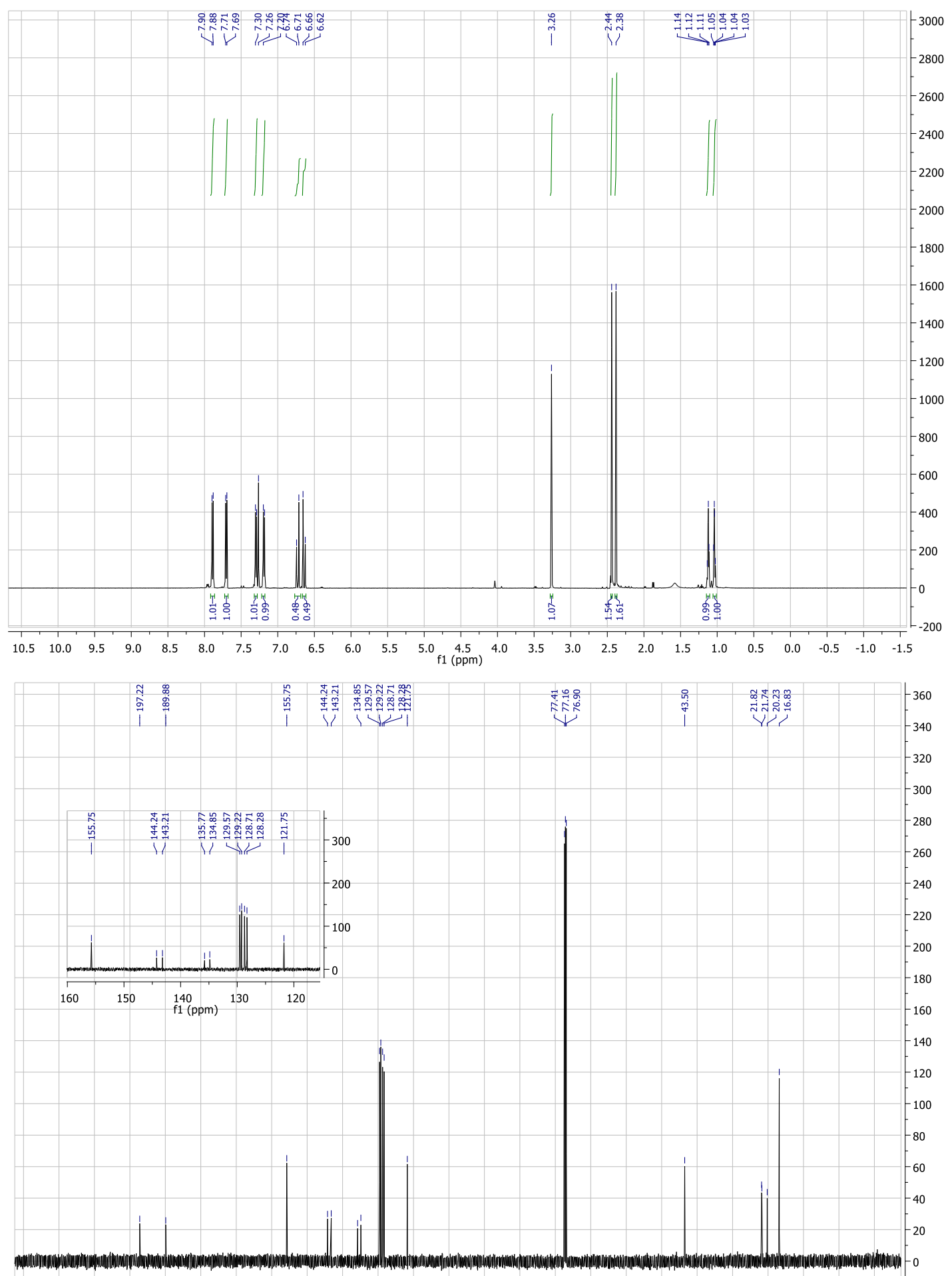

\begin{tabular}{llllllllllllllllllllllllllllll}
\hline 230 & 220 & 210 & 200 & 190 & 180 & 170 & 160 & 150 & 140 & 130 & 120 & $\underset{110}{110} 100$ & 90 & 80 & 70 & 60 & 50 & 40 & 30 & 20 & 10 & 0 & -10
\end{tabular} 
<smiles>COc1ccc(C(=O)/C=C/C2(CC(=O)c3ccc(OC)cc3)CC2)cc1</smiles>
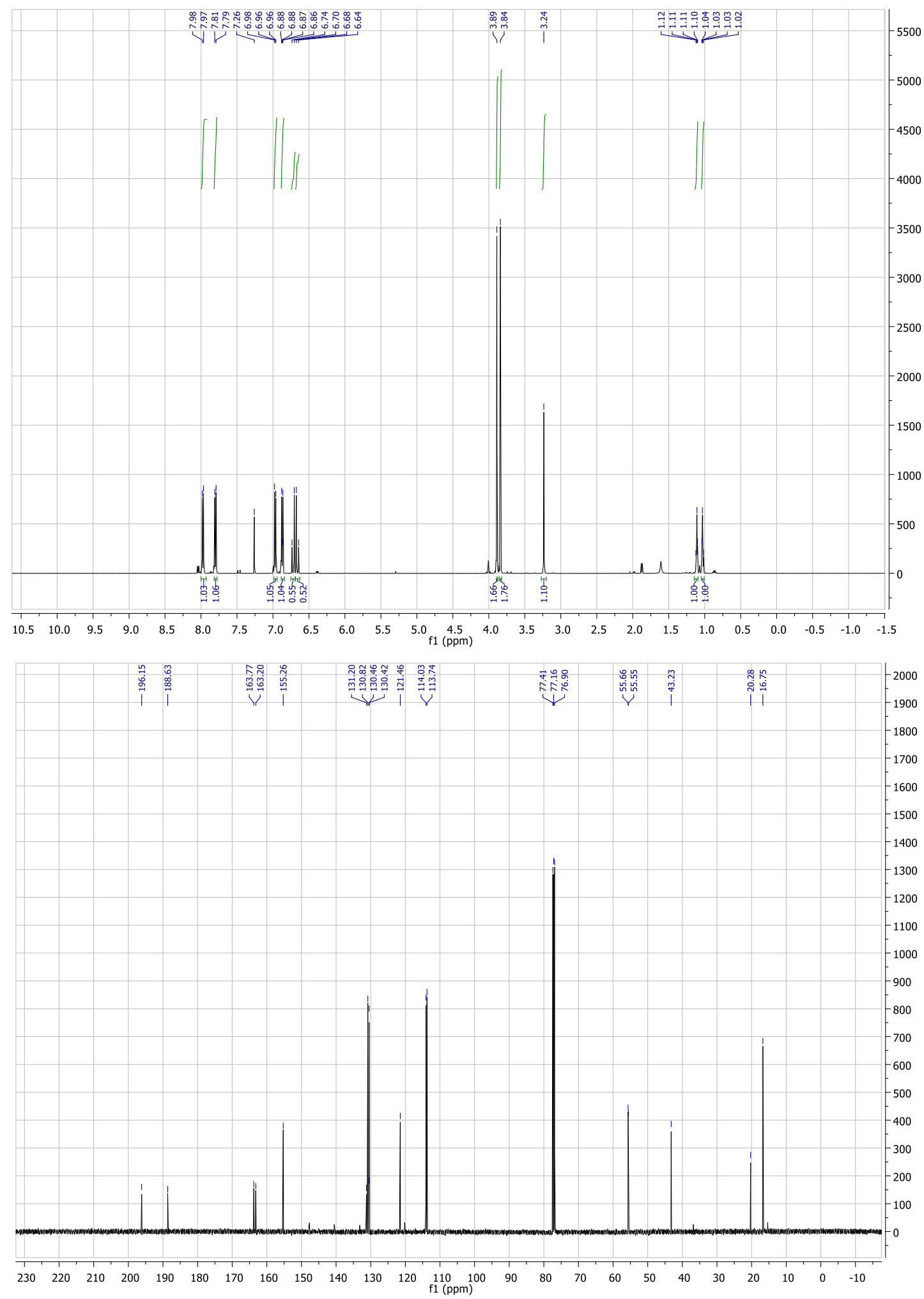
<smiles>O=C(/C=C/C1(CC(=O)c2ccc(Cl)cc2)CC1)c1ccc(Cl)cc1</smiles>
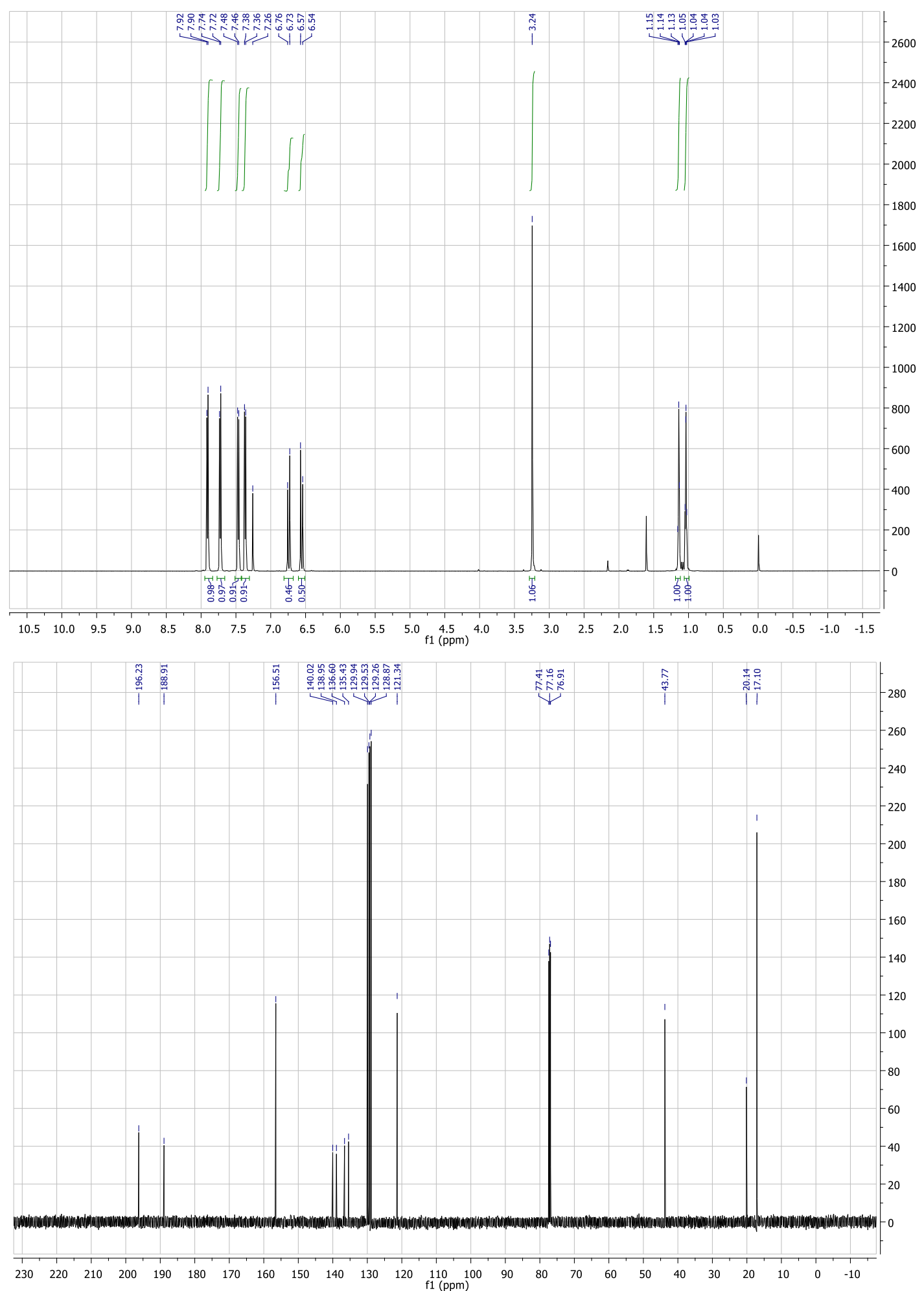

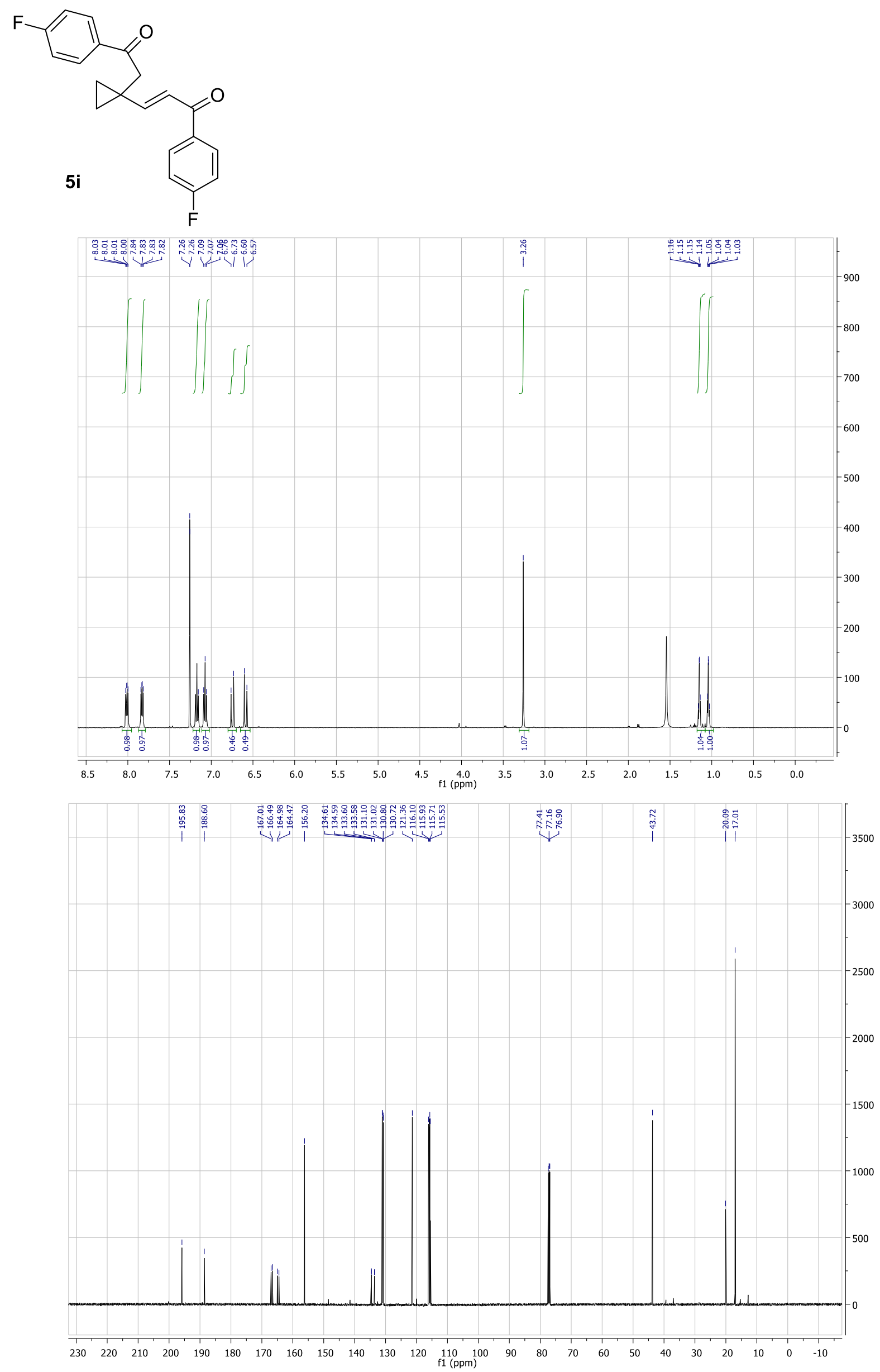

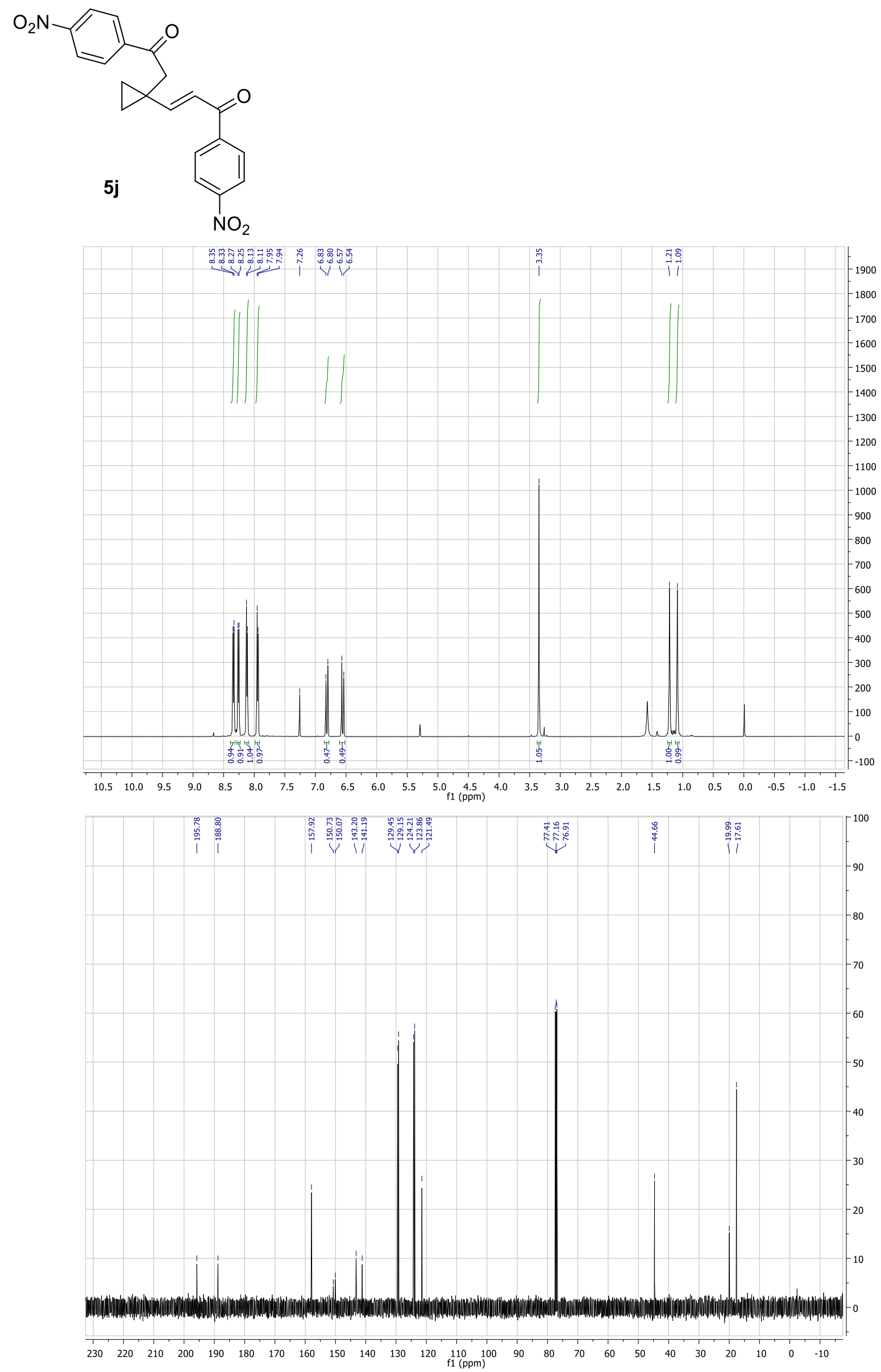

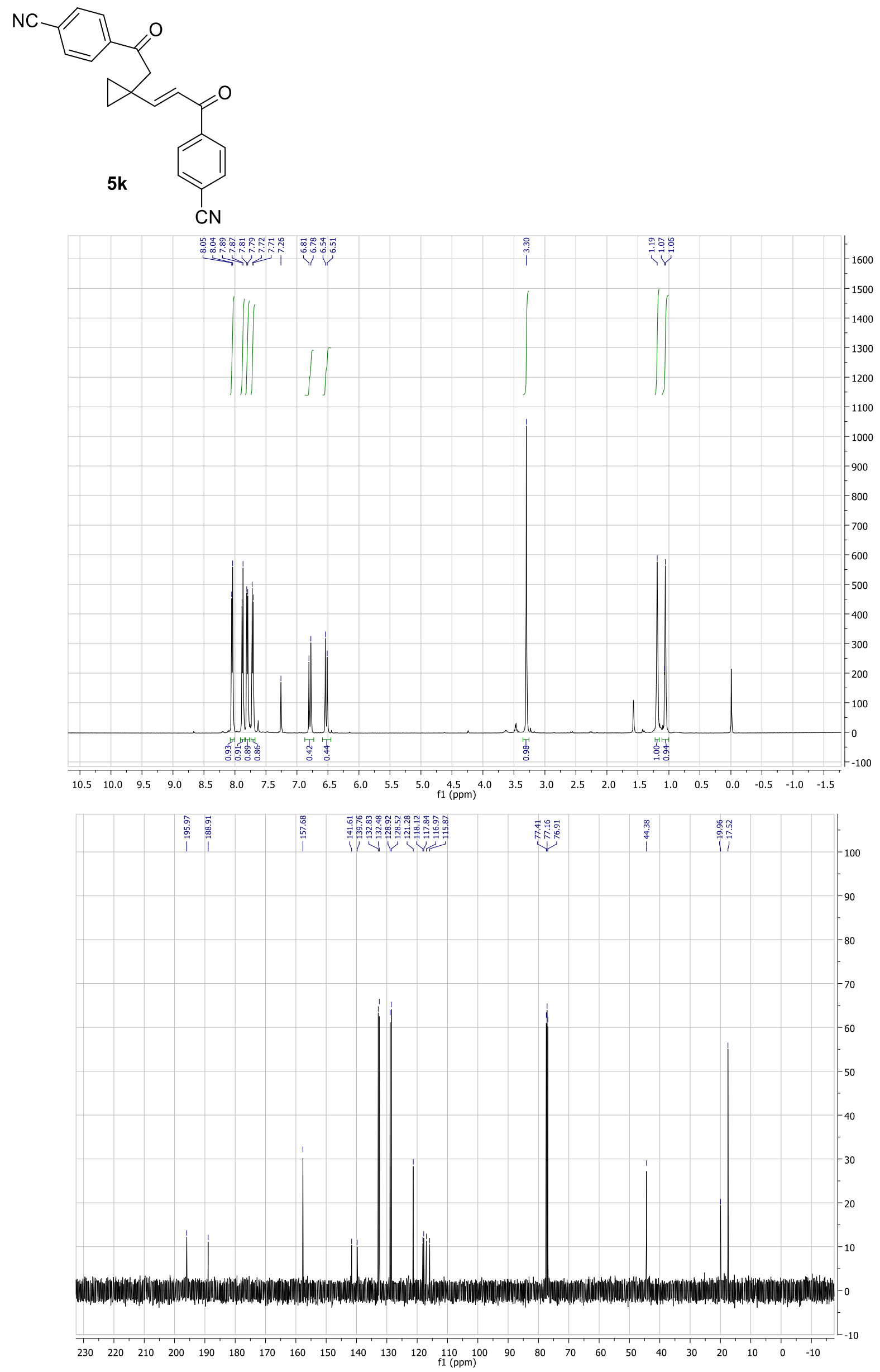
<smiles>CC(=O)C=CC1(CC(C)=O)CC1</smiles>

5s
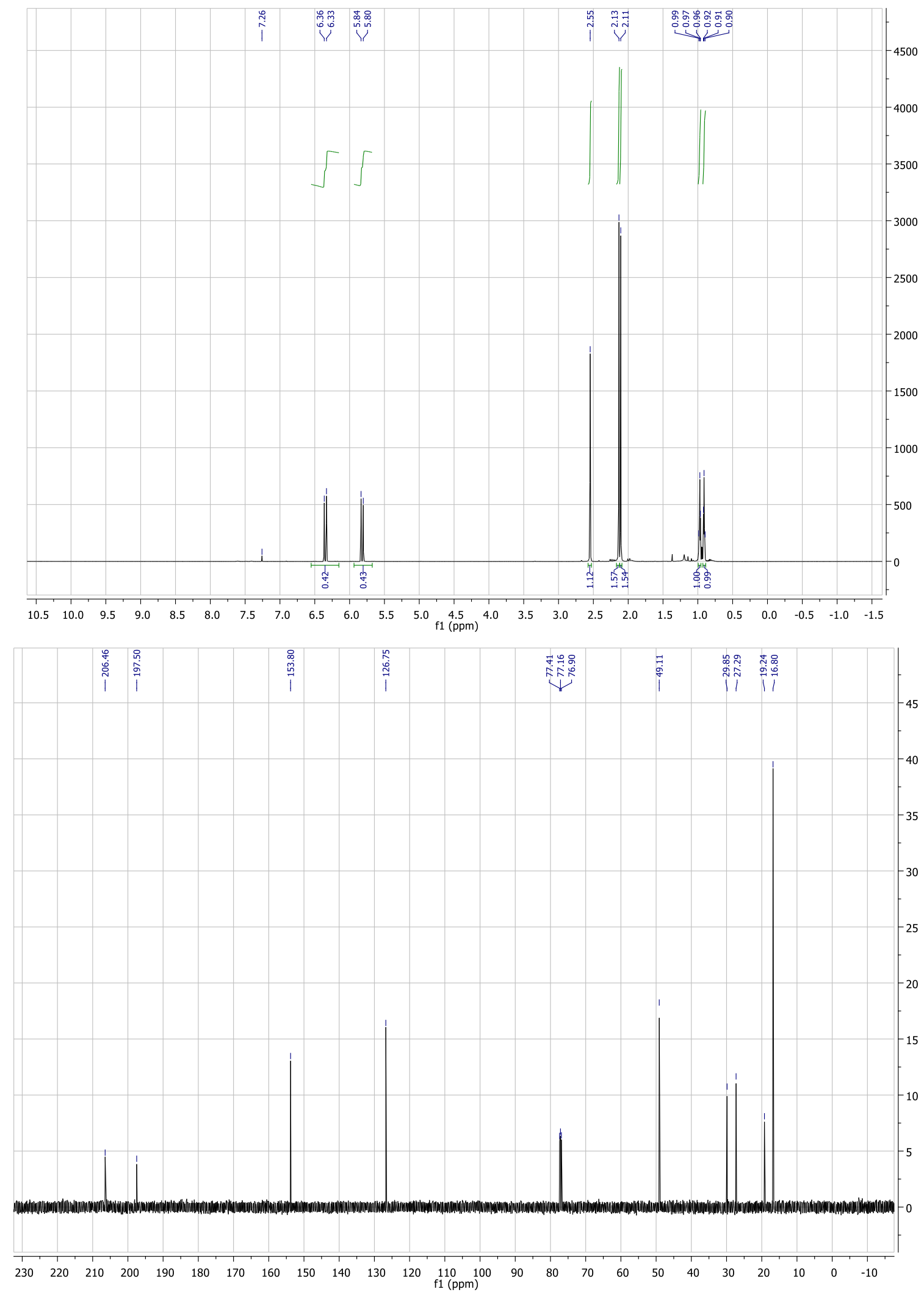

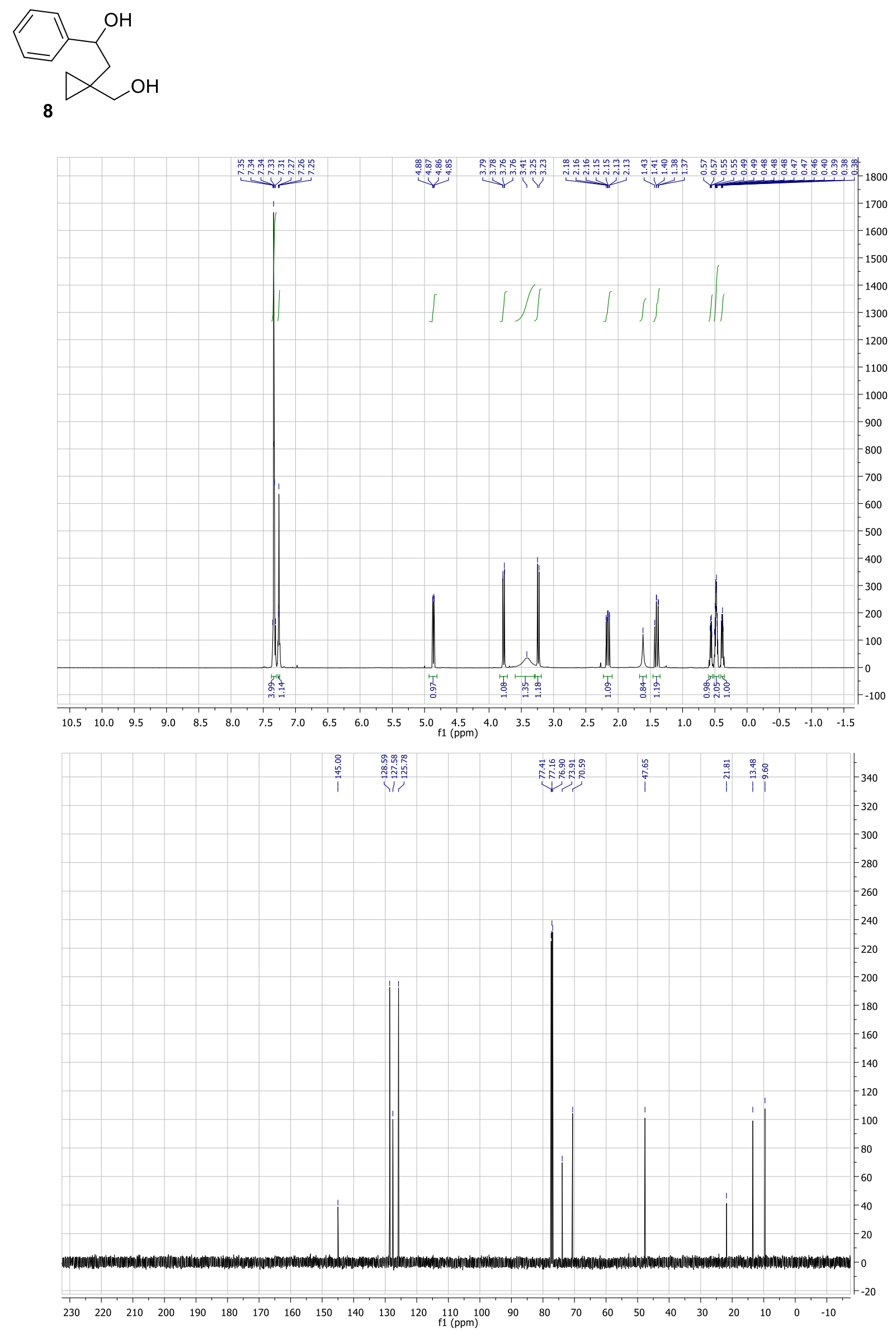


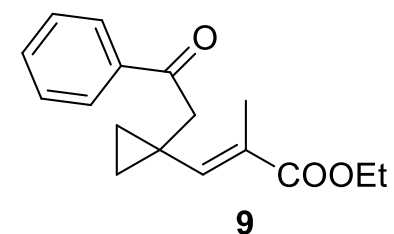

Not separable d.r. 76:24 mixture
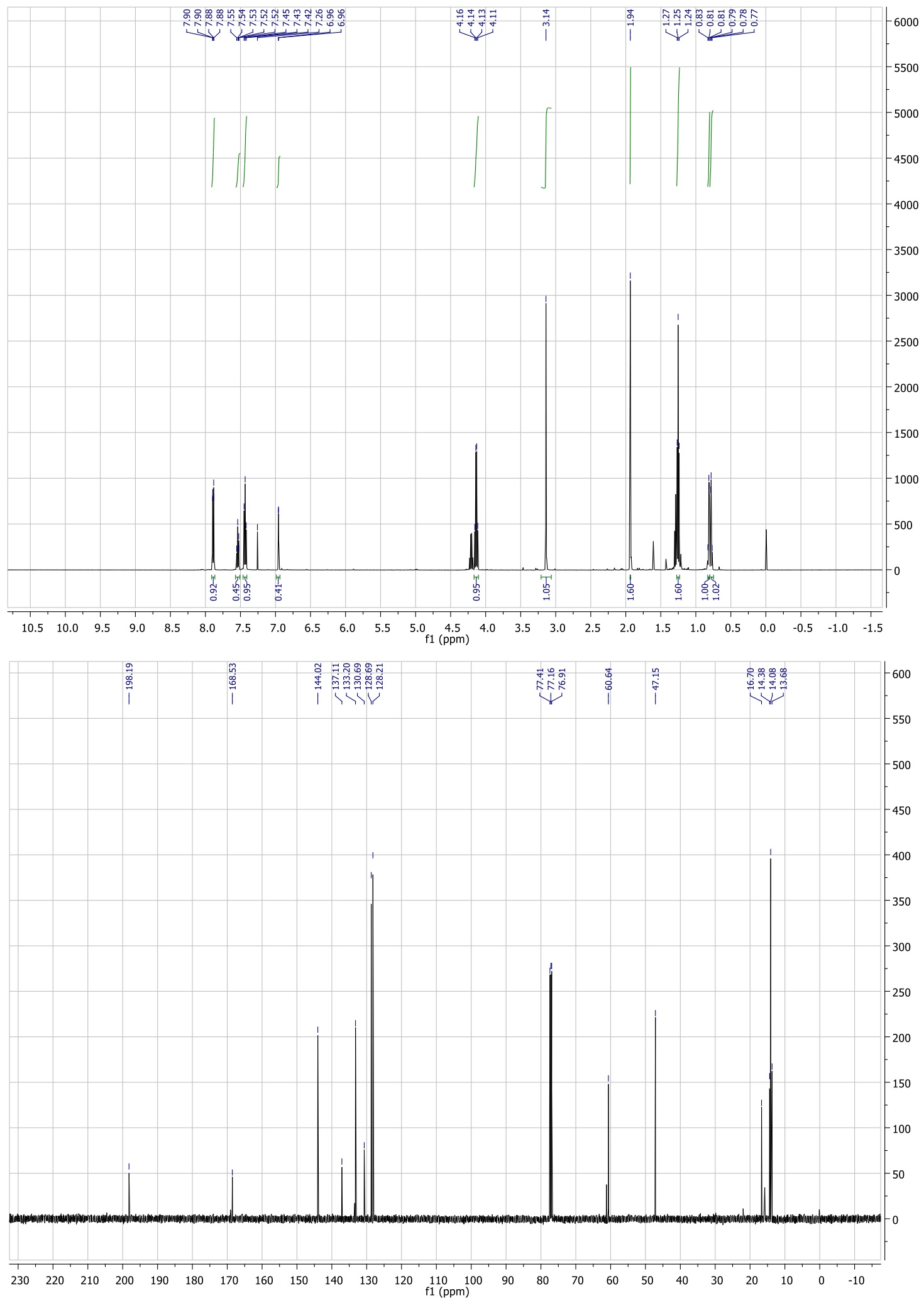


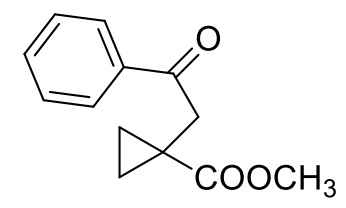

10
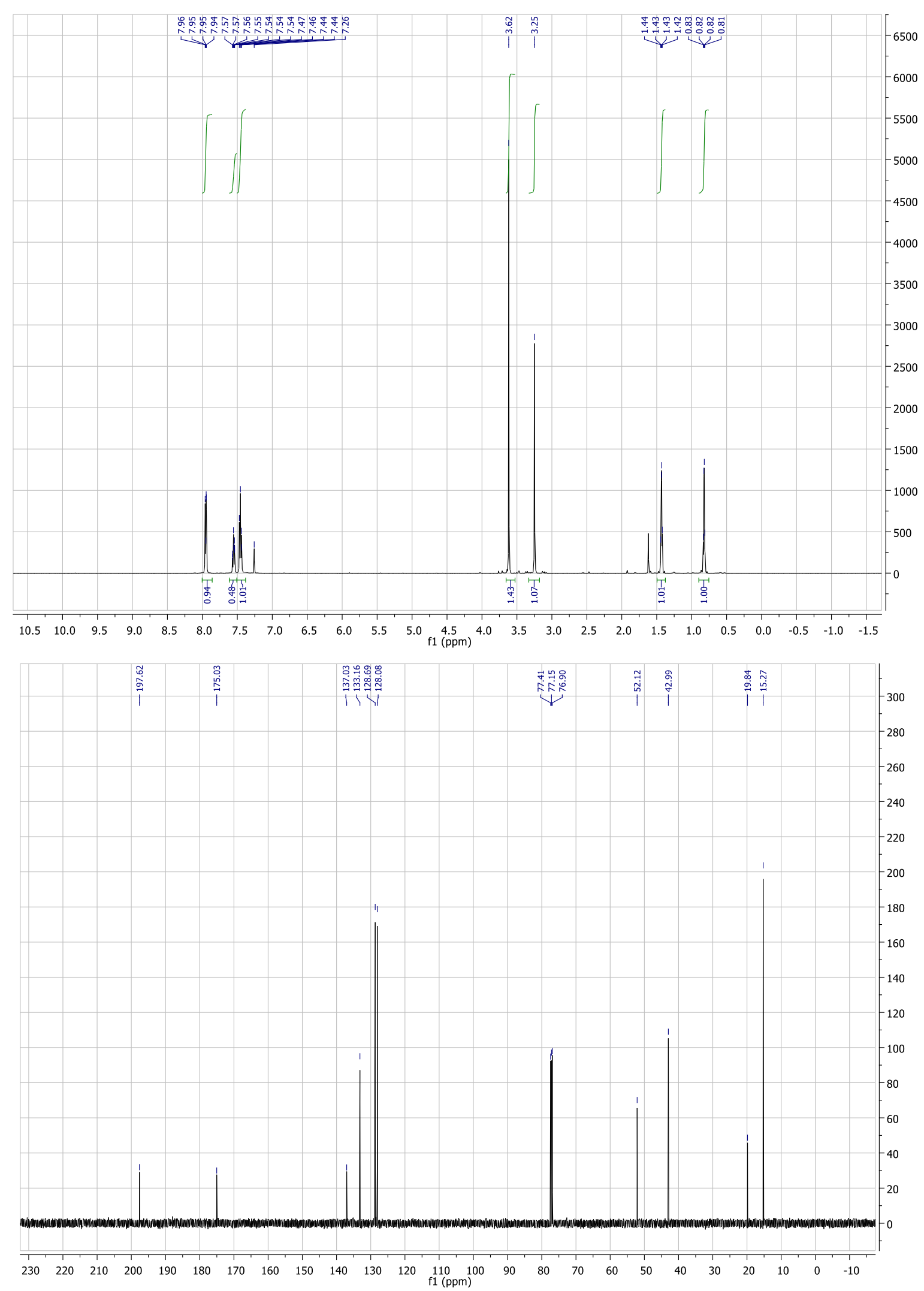


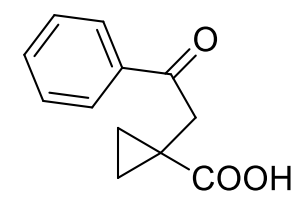

11
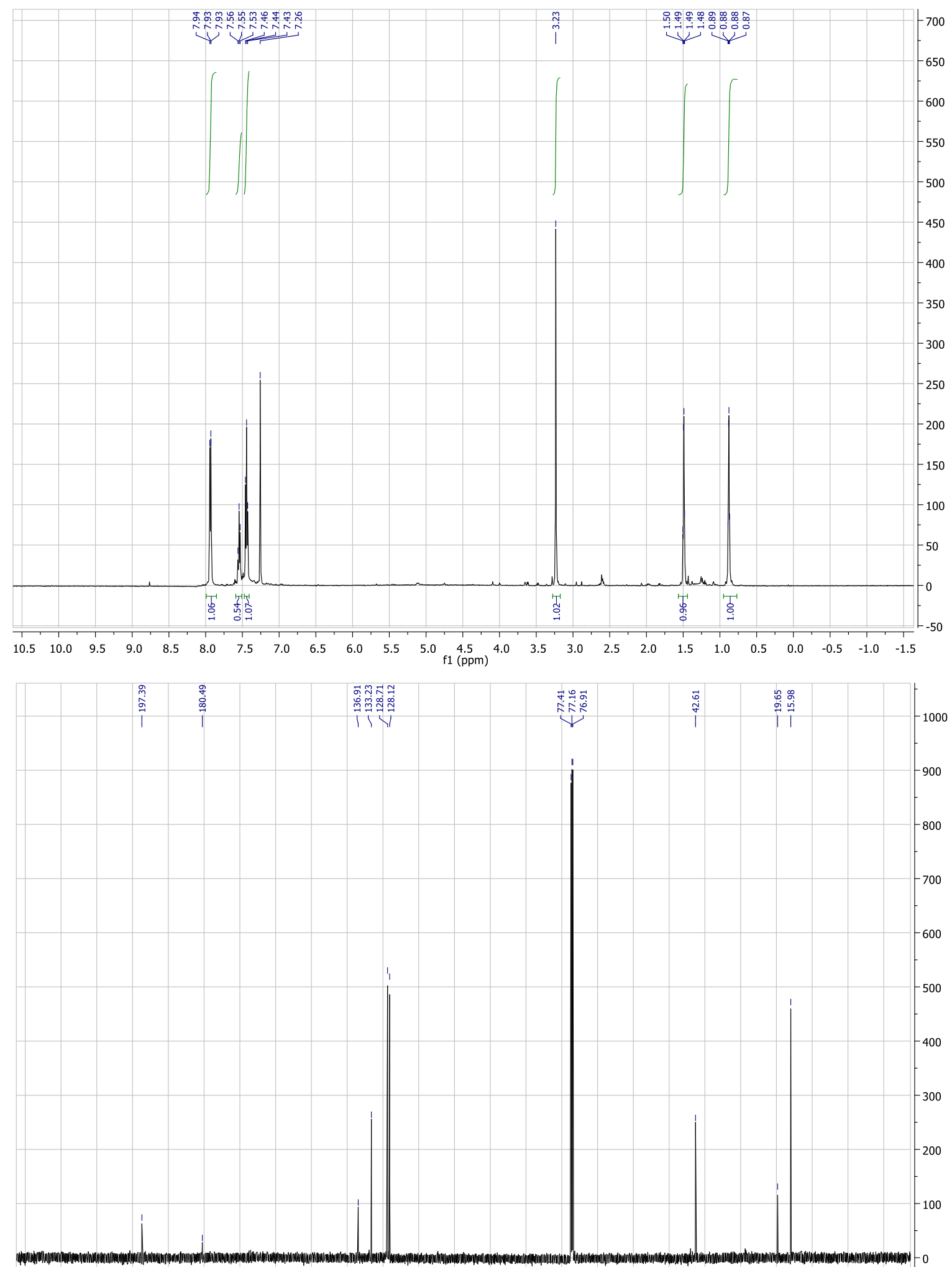

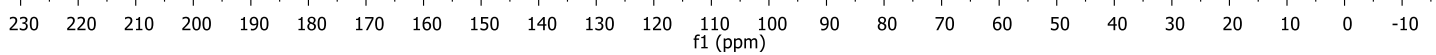




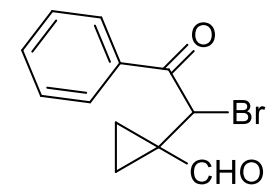

12
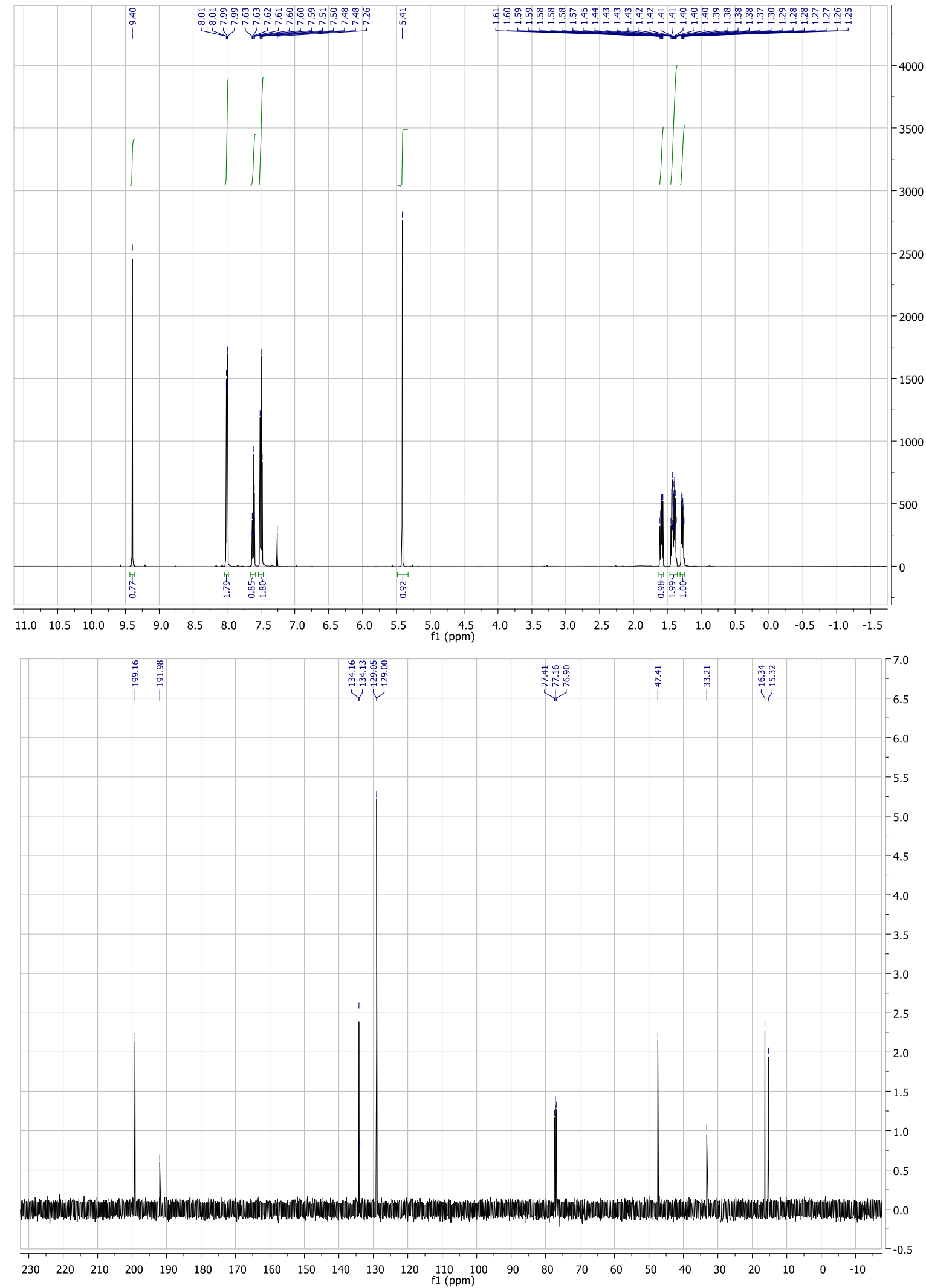